Florida International University FIU Digital Commons

$11-15-2013$

\title{
Towards the Prediction of Mutations in Genomic Sequences
}

Juan Carlos Martinez

jmart054@cs.fiu.edu

DOI: $10.25148 /$ etd.FI13120420

Follow this and additional works at: https://digitalcommons.fiu.edu/etd

Part of the Bioinformatics Commons, and the Computational Engineering Commons

\section{Recommended Citation}

Martinez, Juan Carlos, "Towards the Prediction of Mutations in Genomic Sequences" (2013). FIU Electronic Theses and Dissertations. 987.

https://digitalcommons.fiu.edu/etd/987

This work is brought to you for free and open access by the University Graduate School at FIU Digital Commons. It has been accepted for inclusion in FIU Electronic Theses and Dissertations by an authorized administrator of FIU Digital Commons. For more information, please contact dcc@fiu.edu. 


\section{FLORIDA INTERNATIONAL UNIVERSITY \\ Miami, Florida}

\section{TOWARDS THE PREDICTION OF MUTATIONS IN GENOMIC SEQUENCES}

A dissertation submitted in partial fulfillment of the

requirements for the degree of

DOCTOR OF PHILOSOPHY

in

COMPUTER SCIENCE

by

Juan Carlos Martinez 
To: Dean Amir Mirmiran

College of Engineering and Computing

This dissertation, written by Juan Carlos Martinez, and entitled Towards the Prediction of Mutations in gnomic Sequences, having been approved in respect to style and intellectual content, is referred to you for judgment.

We have read this dissertation and recommend that it be approved.

Jinpeng Wei

Malek Adjouadi

Naphtali Rishe

Shu-Ching Chen

Nelson Lopez-Jimenez

Sundaraja Sitharama Iyengar, Major Professor

Date of Defense: November 15, 2013

The proposal of Juan Carlos Martinez is approved.

$\begin{array}{r}\text { Dean Amir Mirmiran. } \\ \text { College of Engineering and Computing } \\ \hline \begin{array}{r}\text { Dean Lakshmi N. Reddi. } \\ \text { University Graduate School }\end{array}\end{array}$

Florida International University, 2013 
(C) Copyright 2013 by Juan Carlos Martinez

All rights reserved 


\section{DEDICATION}

Dedicated to mom. 


\section{ACKNOWLEDGMENTS}

I would like to dedicate my work to my mother Jene Soto and my mentor Dr. Sitharama Iyengar for their strong and continuing support throughout my academic journey. I would also love to show my huge gratitude to all my FIU committee members: Dr. Chen, Dr. Adjouadi, Dr. Rishe and Dr. Wei for their valuable contribution and advice to my work. My immense gratitude also goes to my non-FIU committee member Dr. Lopez-Jimenez for assisting me with all the insights in the amazing and interesting areas of Medicine and Biology that I needed for my Bioinformatics work. Very special thanks are also given to Dr. Ning Xie who has been my unofficial co-advisor all this time and has provided me with extremely valuable advice. 


\title{
ABSTRACT OF DISSERTATION
}

\section{TOWARDS THE PREDICTION OF MUTATIONS IN GENOMIC SEQUENCES}

\author{
by
}

\section{Juan Carlos Martinez}

Florida International University, 2013

\section{Professor Sundaraja Sitharama Iyengar, Major Professor}

Bio-systems are inherently complex information processing systems. Furthermore, physiological complexities of biological systems limit the formation of a hypothesis in terms of behavior and the ability to test hypothesis. More importantly the identification and classification of mutation in patients are centric topics in today's cancer research.

Many cancers have been traced to somatic mutations in different genes in the genome. Classification of cancer based on gene expression has provided insights into its complex landscape of multiple interactions between gene networks, as well as into possible treatment strategies. Next generation sequencing (NGS) technologies can provide genome-wide coverage at a single nucleotide resolution and at reasonable speed and cost. The unprecedented molecular characterization provided by NGS offers the potential for an individualized approach to treatment. These advances in cancer genomics have enabled scientists to interrogate cancer-specific genomic variants and compare them with the normal variants in the same patient. Analysis of this data provides a catalog of somatic variants, present in tumor genome but not in the normal tissue DNA. Determining the molecular signatures of genes mutated in cancer may help to predict the clinical outcome and carry out therapeutic modifications in treating the patients. However, predicting such signatures at the time of the tumor discovery is a major 
challenge. Several groups have reported lists of predictive genes and reported good predictive performance in terms of prognosis and potential for malignancy based on them. However, the gene lists differed widely and had only very few genes in common. The search for reliable molecular signatures has provided a fertile field for computational approaches. In this dissertation, we present a new computational framework to the problem of predicting the number of mutations on a chromosome for a certain patient, which is a fundamental problem in clinical and research fields. We begin this dissertation with the development of a framework system that is capable of utilizing published data from a longitudinal study of patients with acute myeloid leukemia (AML), who's DNA from both normal as well as malignant tissues was subjected to NGS analysis at various points in time. By processing the sequencing data at the time of cancer diagnosis using the components of our framework which includes training mutations data, mutation extractions, normalization, subspace-based instance filtering, etc., we tested our framework by predicting the regions of the genome to be mutated at the time of relapse and, later, by comparing our results with the actual regions that showed mutations (discovered by sequencing their genomes at the time of relapse). We demonstrate that this coupling of the algorithm pipeline can drastically improve the predictive abilities of searching a reliable molecular signature. Arguably, the most important result of our research is its superior performance to other methods like Radial Basis Function Network, Sequential Minimal Optimization, and Gaussian Process. In the final part of this dissertation, we present a detailed significance, stability and statistical analysis of our model. A comparison performance of the results are presented. This work clearly lays a good foundation for future research for other types of cancer. 


\section{TABLE OF CONTENTS}

CHAPTER

PAGE

1. INTRODUCTION 1

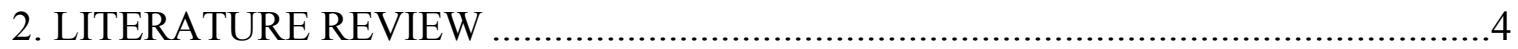

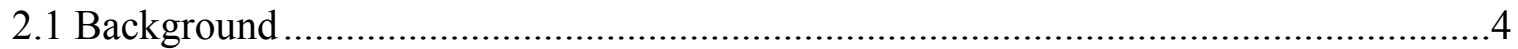

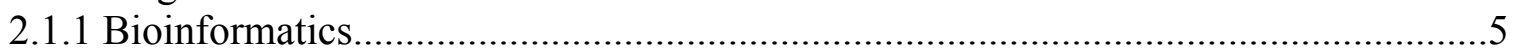

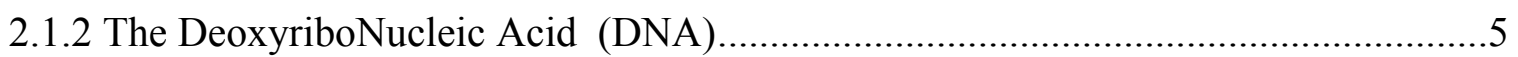

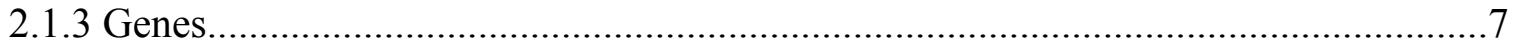

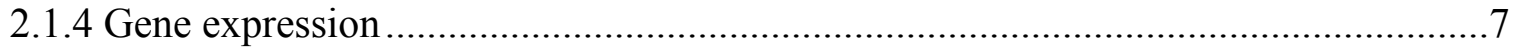

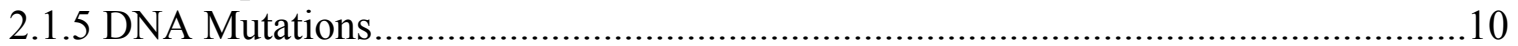

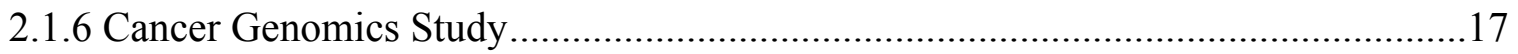

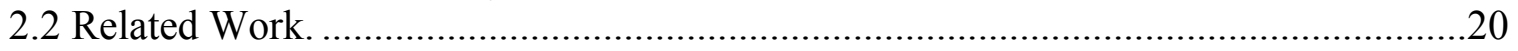

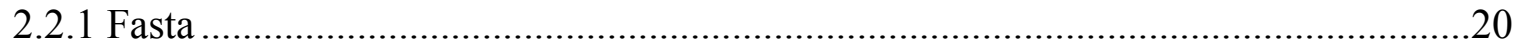

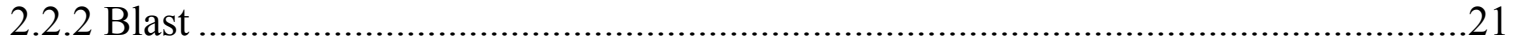

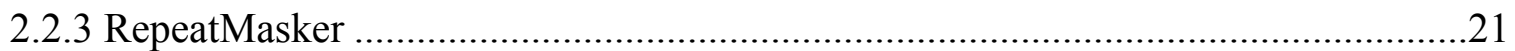

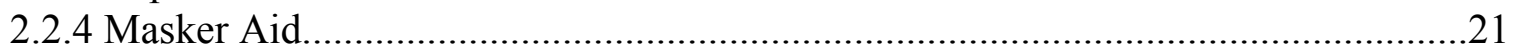

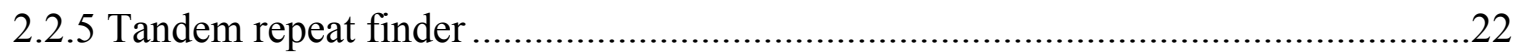

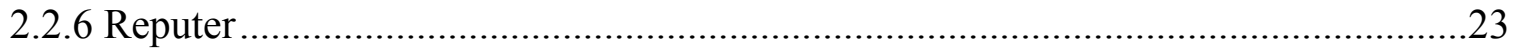

2.2.7 The Vmatch large scale sequence analysis software ...........................................23

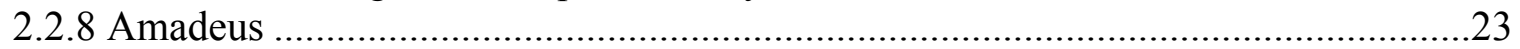

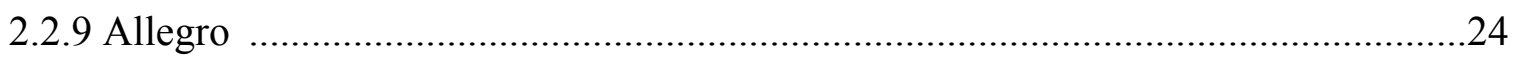

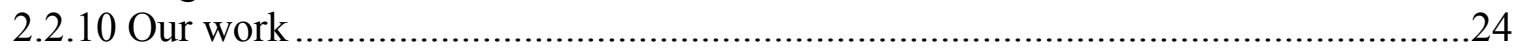

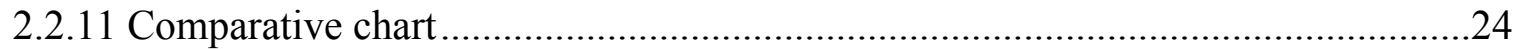

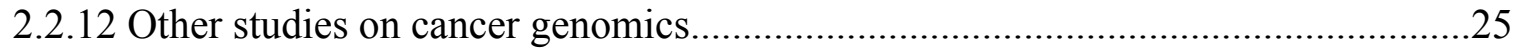

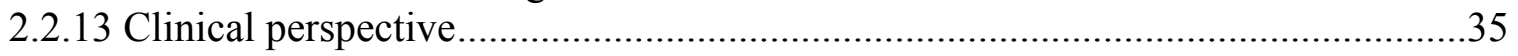

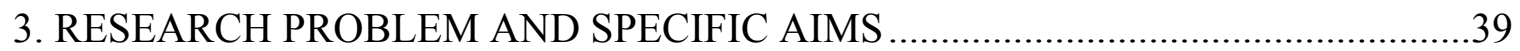

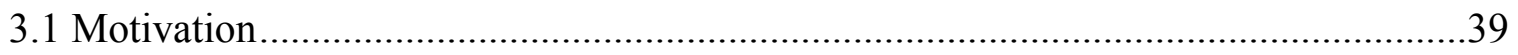

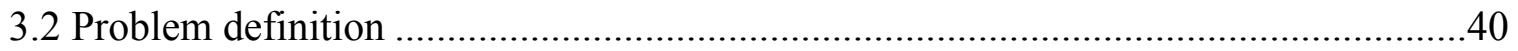

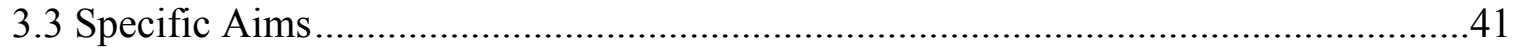

3.3.1 Aim \# 1: To build a tool for statistical analysis on genomic data sets.......................41

3.3.2 Aim \# 2: To utilize this data set and formulate a prediction model...........................41

3.3.3 Aim \# 3: To facilitate the discovery of new functionalities in areas of the genome that had not been previously implicated in cancer........................................................4

3.4 Significance and impact of our work ......................................................................

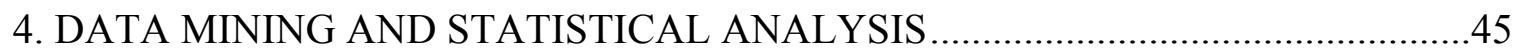

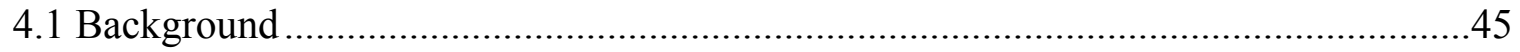

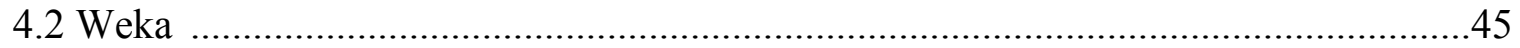

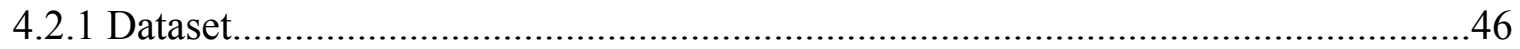

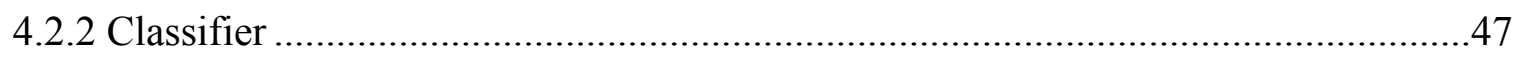


4.2.3 Weka from Java - API ..................................................................................

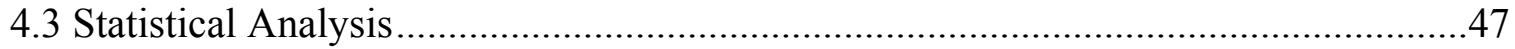

5. MODELING CANCER RELATED GENOME SEQUENCES OF PATIENT DATA50

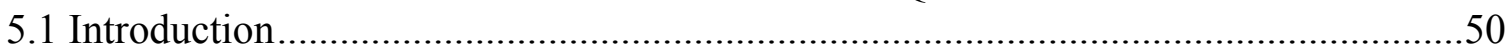

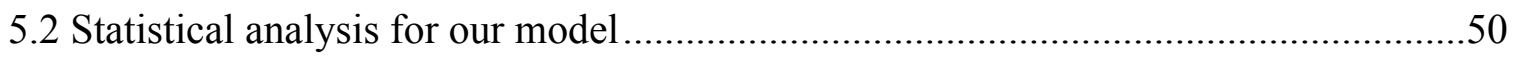

5.3 Mechanisms and Models....................................................................................5

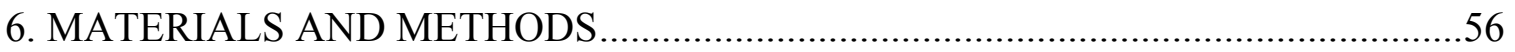

6.1 Framework

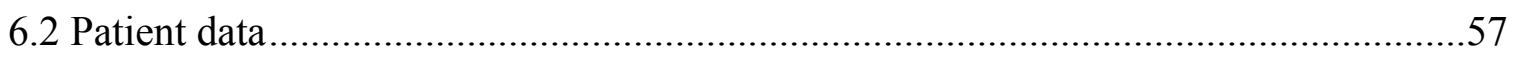

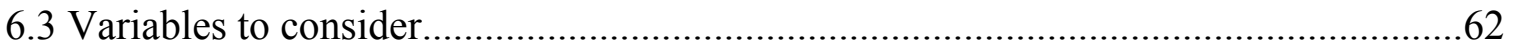

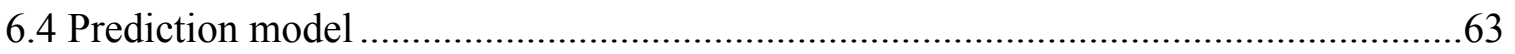

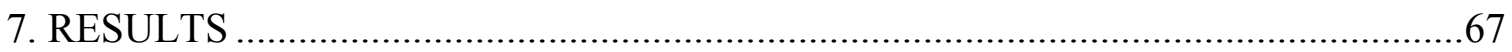

7.1 Prediction of number of mutations per chromosome...............................................67

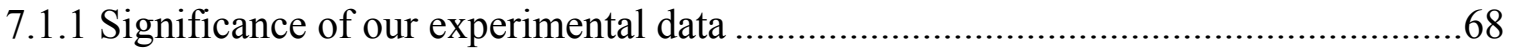

7.1.2 Analysis of performance measures to evaluate the success of our prediction ...........77

7.1.3 Significance, stability and statistical analysis of model.............................................79

7.1.4 Comparison of performance of our model versus other algorithms .........................94

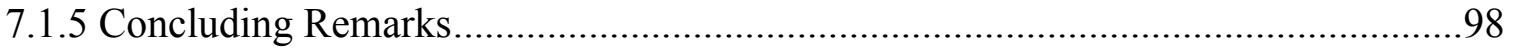

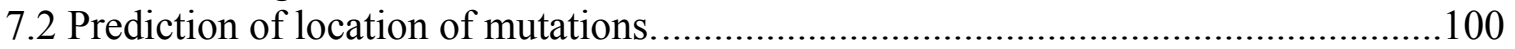

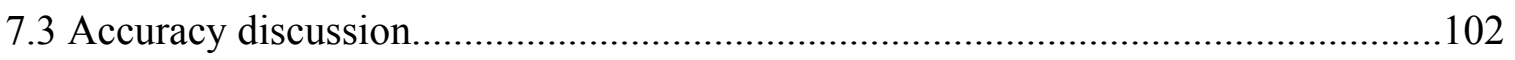

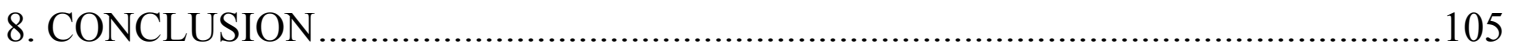

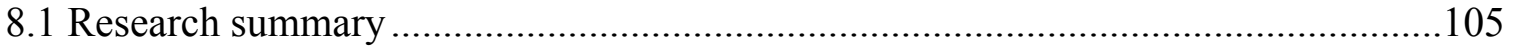

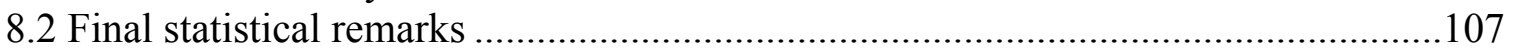

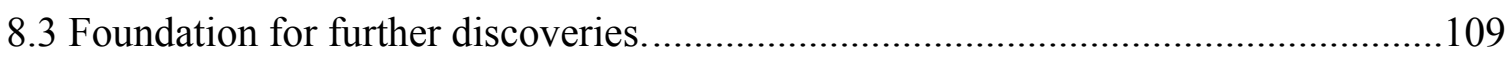

8.4 Application of our framework in the treatment of patients by clinical oncologists...110

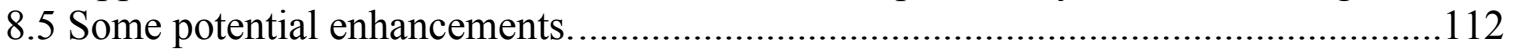

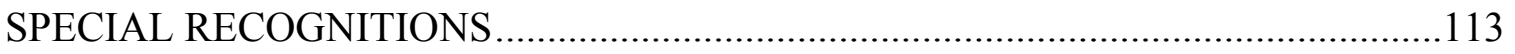

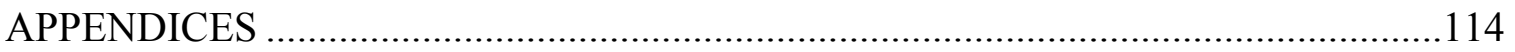

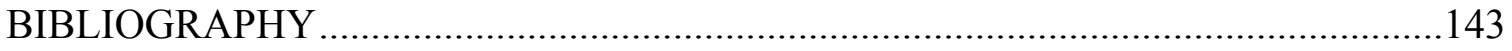

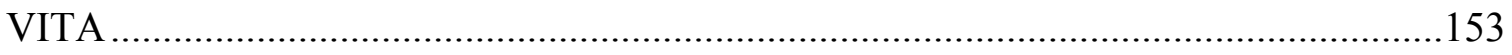




\section{LIST OF TABLES}

TABLE

PAGE

Table 2.1. Examples of genetic diseases caused by expanding tri-nucleotide repeats.

Table 2.2. Pathologic intronic insertions in humans.

Table 2.3. Comparative chart of our work versus other approaches.

Table 2.4. Comparison of Wavelet Analysis and Fourier transform (FT).

Table 6.1. Patient designation, gender and age.

Table 7.1. Number of mutations per chromosome in patient UPN 933124.

Table 7.2. Table for performance measures for our prediction model.

Table 7.3. Statistical analysis of errors for patient A.

Table 7.4. Statistical analysis of errors for patient B.

Table 7.5. Statistical analysis of errors for patient C.

Table 7.6. Statistical analysis of errors for patient D.

Table 7.7. Statistical analysis of errors for patient E.

Table 7.8. Statistical analysis of errors for patient F.

83

Table 7.9. Statistical analysis of errors for patient G.

Table 7.10. Statistical analysis of errors for patient $\mathrm{H}$.

Table 7.11. Statistical analysis of all Patients.

Table 7.12. Coefficients for chromosome models for patient A.

Table 7.13. Coefficients for chromosome models for patient B. 
Table 7.15. Coefficients for chromosome models for patient D.

Table 7.16. Coefficients for chromosome models for patient E.

Table 7.17. Coefficients for chromosome models for patient $\mathrm{F}$.

Table 7.18. Coefficients for chromosome models for patient G.

92

Table 7.19. Coefficients for chromosome models for patient $\mathrm{H}$.

Table 7.20. Comparison table for correlation coefficients for our model versus others.

Table 7.21. Location of mutations, both observed and predicted 102 for patient UPN 933124.

Table 7.22. Summary of results for patient UPN 933124. 


\section{LIST OF FIGURES}

FIGURE

PAGE

Figure 2.1. Graphical representation of a DNA double helix. 6

$\begin{array}{lr}\text { Figure 2.2. Mechanism for gene expression. } & 8\end{array}$

Figure 2.3. Transcription process carried out by DNA 10

polymerase. Blue bases: RNA product.

Figure 2.4. Transcription from DNA to RNA example 10 and translation to protein.

Figure 2.5. DNA Mutation.

$\begin{array}{lr}\text { Figure 2.6. Types of DNA mutations. } & 12\end{array}$

Figure 2.7. Tandem repeat example. 13

Figure 2.8. Transposable element example. 15

Figure 2.9. Sample procedure of applying wavelet transform 28

in cancer genome analysis.

Figure 2.10. The four types of mutations. 31

Figure 6.1. Dependency flow graph for our prediction framework. 58

Figure 6.2. Sample data files for patient UPN 933124. 62

$\begin{array}{ll}\text { Figure 6.3. Overview of geneMap.txt. } & 62\end{array}$

Figure 6.4. Weka input file. $\quad 64$

Figure 6.5 Algorithm for finding mutations locations. 66

Figure 7.1 Histogram of absolute error for patient A. 69

Figure 7.2 Histogram of absolute error for patient B. 69

$\begin{array}{ll}\text { Figure 7.3 Histogram of absolute error for patient C. } & 70\end{array}$

$\begin{array}{ll}\text { Figure 7.4 Histogram of absolute error for patient D. } & 70\end{array}$ 
Figure 7.5 Histogram of absolute error for patient E.

Figure 7.6 Histogram of absolute error for patient F.

Figure 7.7 Histogram of absolute error for patient G.

Figure 7.8 Histogram of absolute error for patient $\mathrm{H}$.

Figure 7.9 Histogram of relative error for patient A.

Figure 7.10 Histogram of relative error for patient B.

Figure 7.11 Histogram of relative error for patient C.

Figure 7.12 Histogram of relative error for patient D.

Figure 7.13 Histogram of relative error for patient E. 75

Figure 7.14 Histogram of relative error for patient F. 75

Figure 7.15 Histogram of relative error for patient G. 76

Figure 7.16 Histogram of relative error for patient $\mathrm{H}$. 76

Figure 7.17 Comparison of performance of our model versus others. $\quad 98$

Figure 7.18 Potential estimation error graph as more data is obtained.

100 


\section{ACRONYMS AND ABBREVIATIONS}

$\begin{array}{ll}\text { DNA } & \text { Deoxyribonucleic Acid } \\ \text { A,C,T,G } & \text { Adenine (A), cytosine (C), guanine (G), and thymine (T) } \\ \text { RNA } & \text { Ribonucleic Acid } \\ \text { mRNA } & \text { Messenger RNA } \\ \text { TR } & \text { Tandem Repeat } \\ \text { TE } & \text { Transposable Element } \\ \text { NCBI } & \text { National Center for Biotechnology Information } \\ \text { NGS } & \text { Next Generation Sequencing } \\ \text { OO } & \text { Object Oriented } \\ \text { ML } & \text { Machine Learning } \\ \text { IDE } & \text { Integrated Development Environment } \\ \text { JDK } & \text { Java Development Kit }\end{array}$




\section{CHAPTER 1}

\section{INTRODUCTION}

In academia, as well as in industry, there have been a number of efforts in developing computer algorithms to aid the research in molecular biology. Due to the fact that both DNA and proteins can be directly represented as a sequence of characters (either bases or aminoacids, respectively), the bioinformatics field has come up with a large number of computer solutions with a strong emphasis on string algorithms. A significant number of studies has shown that cancer development brings about changes in DNA molecule at a nucleotide level (mutations) within the genome of an individual.

Many cancers have been traced to somatic mutations in different genes in the genome. [1]. The classification of cancer based on gene expression has provided much insight into the complex landscape of multiple interactions between gene networks, as well as into possible treatment strategies. Advances in cancer genomics have been accelerated with the emergence of high-throughput sequencing strategies that enable scientists to interrogate cancer-specific genomic variants and compare them with the normal variants in the same patient [2][3]. Next generation sequencing (NGS) technologies can provide genome-wide coverage at a single nucleotide resolution and at reasonable speed and cost. [4]. The unprecedented molecular characterization provided by NGS offers the potential for an individualized approach to treatment.

One of the contributions of such strategies includes the definition of relatively characteristic gene expression profiles, or molecular signatures that may have prognostic 
implications for targeted therapies. In cancer patients, the objective of NGS is to obtain and compare information about cancer and normal tissue DNAs. Analyses of the data provide a catalog of somatic variants present in tumor genome but not in the normal tissue DNA. The goal of the analysis is to reveal a putative drug target in the examined cancer which facilitates the selection of therapy, and improves the personalized risk assessment. Moreover, determining the molecular signatures of genes mutated in cancer may help to predict the clinical outcomes [5].

Predicting molecular signatures based on the initial events in cancer development may help researchers, as well as clinicians, to carry out therapeutic modifications in treating the patients. However, predicting such signatures at the time of the tumor diagnosis is a major challenge. Several groups have reported lists of predictive genes and reported good predictive performance in terms of prognosis and potential for malignancy based on them. However, the gene lists differed widely and had only very few genes in common. Furthermore, those types of predictions were based on computational approaches not involving NGS, such as microarray analysis, $\mathrm{qPCR}$, and others [6][7][8] in various types of cancer such as colorectal [9], lung [10], prostate[11], breast cancer[12] and others. Additionally, the disadvantages of current predictive models in cancer are that they are focused on evaluating mutations that anticipate the risk of progression and its clinical impact on the length of patient's survival. They are not intended to predict mutational events at molecular level, rather to detect and classify existing mutations, and utilize that information to make predictions in the behavior of the cancer disease.

In our work, by having the full sequence of the genome from both normal and cancer tissue at various points in time, we are able to extract valuable information to validate the 
predictions generated from the genome. Thus, we propose to search for longitudinal studies of cancer patients with such identified mutated genes by the use of NGS at different points in time. The mutation profile identified earlier in time will serve as the basis for the prediction later in time. Furthermore, we will compare the observed mutated locations at the time of relapse with the results of our own predictions.

Our goal in this study was to test a computational framework utilizing published data from a longitudinal study [13] of patients with acute myeloid leukemia (AML) whose DNA from both normal as well as cancer tissues were subjected to next generation sequencing analysis at various points in time. First of all, we processed the longitudinal sequencing data from cancer tissue. Secondly, we tested our framework by predicting the regions of the genome to mutate at the time of relapse. Finally, we compared our results with the observed mutated regions, identified by sequencing their genomes at the time of relapse. The accuracy of the predicted number of mutations ranged from 75 to $84 \%$; the accuracy of the locations of mutations ranged from $69 \%$ to $88 \%$ in the patients analyzed. Our predictions agree well with the reported data. Although our approach is purely computational and does not provide insights into the putative molecular mechanisms by which the mutations occur, we are confident that it may assist researchers in determining the importance of a particular mutation in cancer progression, thus providing another tool to select candidates to target in drug development for cancer treatment. 


\section{CHAPTER 2}

\section{LITERATURE REVIEW}

\subsection{Background}

Over time our knowledge of genomes has developed at the same rate as their sequencing. Furthermore, sequencing capacity has grown significantly recently. The characterization of the genome starts from its sequencing or reading step and assembling. However, it does not end by the time its sequence is obtained. Thus, at this point, we are just at the beginning of a much more complex study. We are constantly looking for better coverage of the sequence and better resolution and, as a result, the assembly of the human genome is a multistep always-evolving process. The Genome Reference Consortium frequently produces a new version of the human reference genome for scientists to have a unique set of gene coordinates when comparing their results. The latest release from GRC is the $37^{\text {th }}$ [14]. With every new release, a large set of analysis is required to understand the works of the genome, its functionalities, and how we can detect any anomalies in it as well as how we can predict future ones.

Nevertheless, before going into further details of how to understand the scope of our research of the genome, it is necessary to have a minimum background on how it works [111]. In the next pages, we will provide some basic concepts in the Biology field that will ease the understanding of the rest of the work. This dissertation was written for readers who do not necessarily have the biology background, and, as result, a minimal background is provided. 


\subsubsection{Bioinformatics}

Bioinformatics is a compound word that consists of two root words. The first word "bio" indicates we are dealing with problems that originate from biology. The second word "informatics" indicates that the problems are to be solved by the discipline that works on the management of information and the design of computational algorithms, namely, computer science.

\subsubsection{The DeoxyriboNucleic Acid (DNA)}

The DeoxyriboNucleic Acid (DNA) [16][17] plays the role of the information archive for all organisms. DNA molecules present a double helix structure containing four-letter bases which stand for the 4 nucleotides. These four nucleotides are adenine (A), cytosine $(\mathrm{C})$, guanine $(\mathrm{G})$, and thymine $(\mathrm{T})$. Nucleotides belong to two types: purines and pyrimidnes. Adenine and guanine fall into the first category while cytosine and thymine into the second one.

DNA is not normally present as a single long molecule but as an associated pair of molecules. DNA strands present the shape of a double helix as can be seen in Figure 2.1. Alternating phosphate and sugar elements compose the backbone of a DNA strand. The direction of the nucleotides in one strand is always opposite to the direction from the other. As a result, we consider DNA strands as anti-parallel. These DNA strands are asymmetric and known as 5' and $3^{\prime}$ and follow a direction from one to the other. All bases on one strand form a bond with specifically one type of base on the other strand. We call this behavior complementary base pairing. Thus, purines form hydrogen complement with pyrimidines: A with $\mathrm{T}(\mathrm{A}=\mathrm{T}$ or $\mathrm{T}=\mathrm{A})$, and $\mathrm{C}$ with $\mathrm{G}(\mathrm{C}=\mathrm{G}$ or $\mathrm{G}=\mathrm{C})$. When two 
nucleotides are bound together across the DNA strands form base pair (bp).

The DNA presents a double stranded helix with complimentary information on each strand. This characteristic is of the DNA is vital in the functionality of the DNA for all living beings [18]. DNA has mechanisms for self-replication and for translation of genes into proteins. Furthermore, replications are necessary for the stability of the inheritance. However, some imperfect replications are also necessary for evolution. Genetic information is implemented by the synthesis of mRNA (messenger RNA) into proteins. Proteins are molecules in charge of the structure and activities of living beings. Antibodies, produced by our own organisms, to fight disease, along with some enzymes and muscle tissue belong to the protein category. Proteins can be composed of 200-400 amino acids long, requiring 600-1200 DNA bases. Surprisingly, only a small percentage of the total sequence genome encodes for proteins. There are vast areas of the DNA sequence which account for control mechanisms, and others for which no functionality has been discovered yet, and, therefore, considered as "junk". However, it might not necessarily be junk as there have been discoveries of transposable elements that have a regulatory function in this area, leaving us with more to be discovered in these areas [19][20].

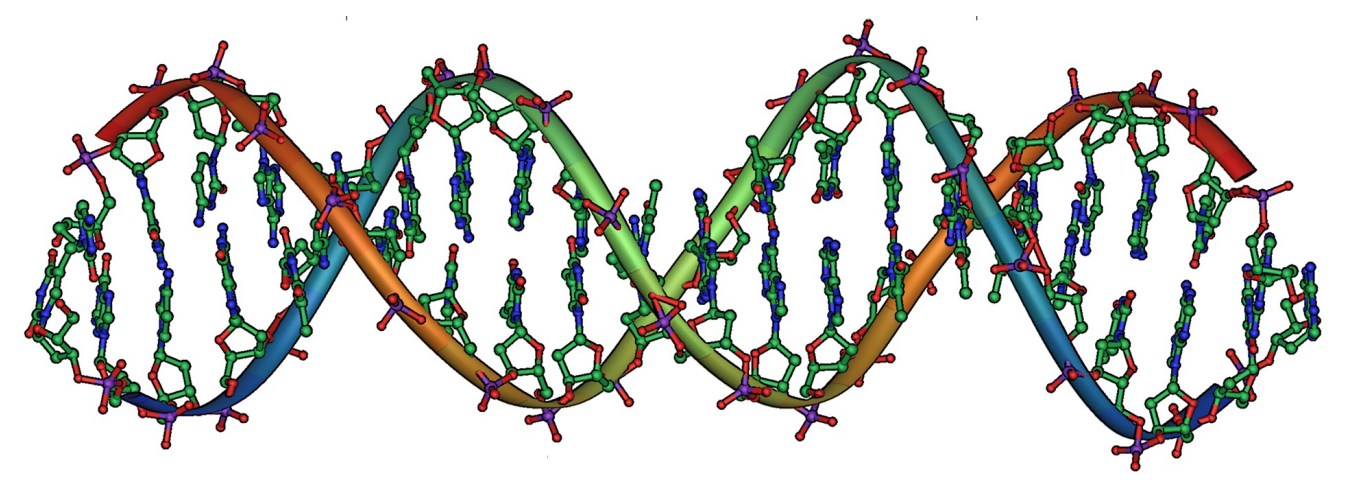

Figure 2.1. Graphical representation of a DNA double helix. [111] 


\subsubsection{Genes}

Our main unit of heredity for all organisms is called a gene. Genes keep the necessary information to build and transfer genetic traits to descendants as well as to maintain cells. Simply speaking, a gene can be seen as a segment of nucleic acid that, when considered as a whole, specifies a trait. Genes can be mapped to multiple biological traits such as such as eye or hair color.

Genes have coding sequences or exons and non-coding sequences or introns. If a gene is active, both exons and introns are copied though transcription, producing as an output an RNA copy of the information from the gene. The output allows for protein synthesis from genetic code. The molecules that are produced from gene expression are called gene products and are the ones that make it possible for organisms to develop proper functionality.. Gerstein et al. [21] provide a precise definition of a gene: "A gene is a union of genomic sequences encoding a coherent set of potentially overlapping functional products". Besides the regions that code for protein, genes also have regulatory regions as can be seen in Figure 2.2.

Regulatory regions include enhancers that are responsible for compensating for a weak promoter [22]. Small RNAs consist of short sequences roughly between 18 and 25 bases. They act as regulators of stability or availability for translation of RNAs.

\subsubsection{Gene expression}

When a gene is expressed, the products obtained from it are often proteins. Gene expression is a widely used process for all organisms ranging from the most basic ones to the most complex and developed ones. 
Gene regulation allows cell function and control over structure and, as a result, is the base for cellular differentiation and adaptability. Gene expression is the main level where the genotype gives rise to the phenotype. Thus, genetic code can be interpreted.

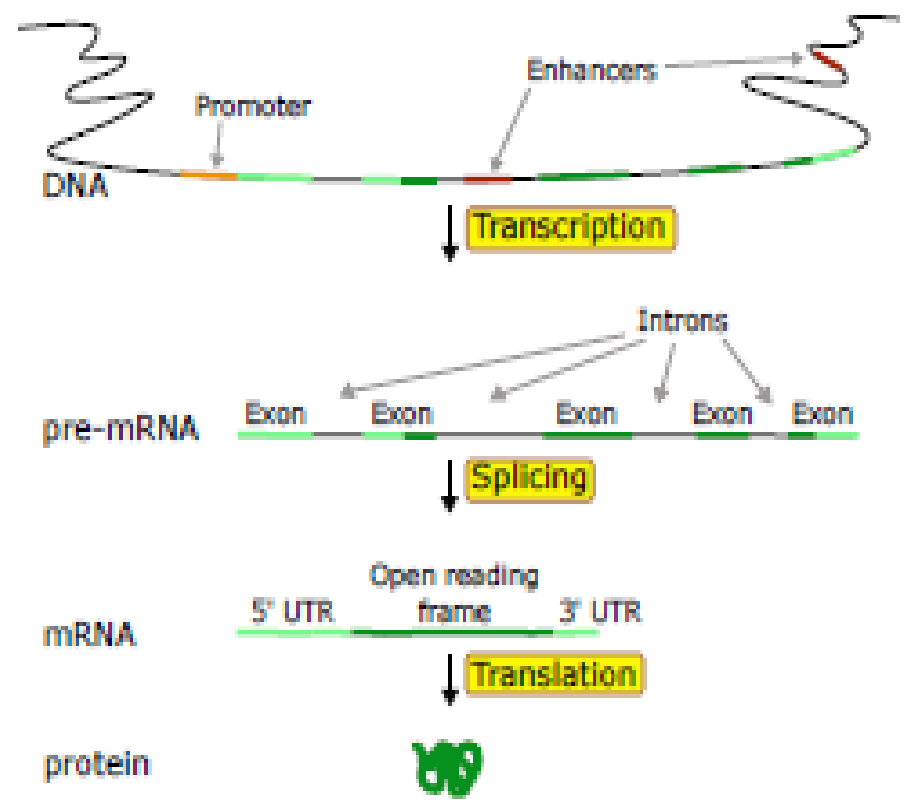

Figure 2.2. Mechanism for gene expression. [15]

Gene expression is divided into 5 steps:

\section{i. Transcription}

The gene acts as a blueprint for the production of RNA. We call transcription to the RNA's production copies of the DNA. It is done by RNA polymerase by adding one RNA nucleotide at a time to a growing RNA strand as can be observed in Figure 2.3. RNA complementarity to DNA is present here. For instance, a $\mathrm{T}$ in the DNA will 
produce an A in the RNA. Nevertheless, in RNA the Uracil is obtained instead of Thymine in place of an Adenine in the DNA strand. For instance, the mRNA complement of a DNA strand piece "CAT" would be transcribed as "GUA". Figure 2.4 shows more on this.

\section{ii. $R N A$ processing}

Even though, it is not the only, splicing to remove introns is the most common RNA processing.

\section{iii. RNA export}

In eukaryotes, mature RNA has to be exported out of the nucleus to the cytoplasm which is where proteins actually work [15].

\section{iv. Translation}

Every RNA triplet has a corresponding binding site for amino acids which are chained together by the ribosome. The ribosome produces a structure-less protein out of the by taking the amino acid from each transfer RNA. The second step of the Figure 2.4 illustrates this process.

\section{v. Folding}

Here, the recently formed unfolded polypeptide will proceed to fold into the functional 3dimensional structure. 
After this process, the protein working into the cell or transported outside of it as it occurs in digestive enzymes.

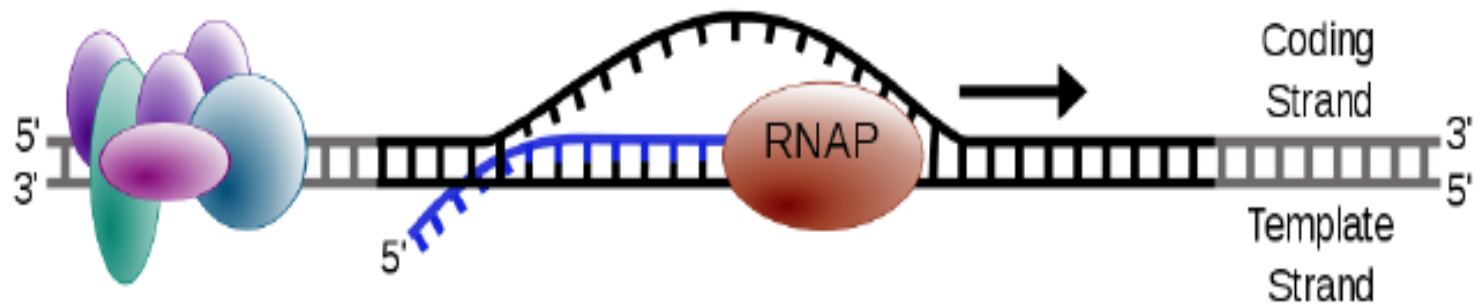

Figure 2.3. Transcription process carried out by DNA polymerase. Blue bases: RNA product.[15]

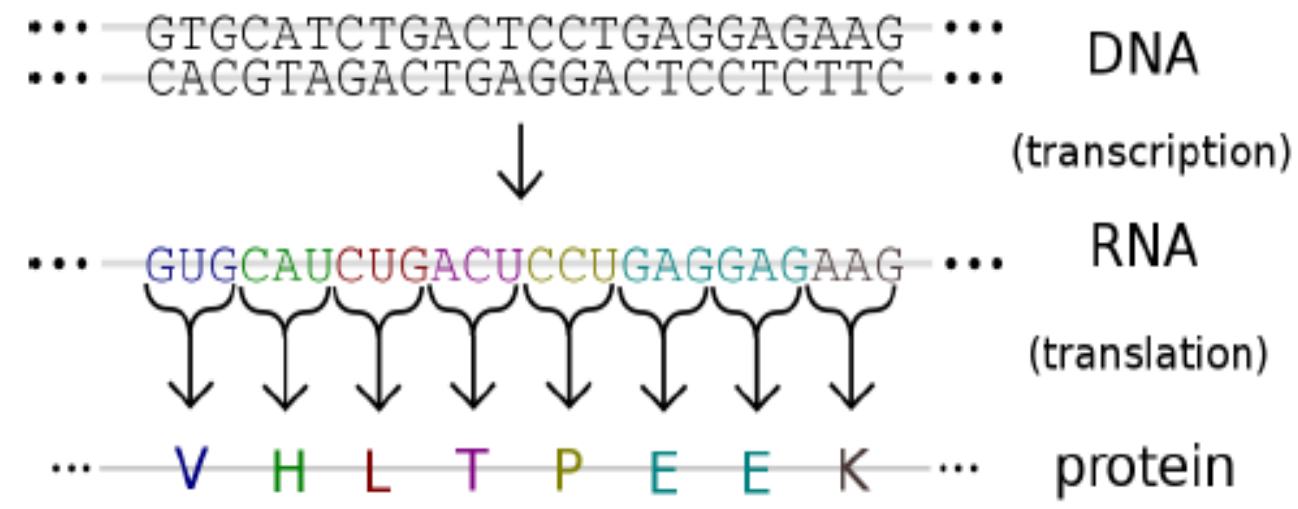

Figure 2.4. Transcription from DNA to RNA example and translation to protein. [15]

\subsubsection{DNA Mutations}

We define DNA mutations as the sequences that appear within a genome that do not 
comply with what is "expected" in that location. The human DNA is very similar from individual to individual with minor differences in the nucleotides that appear in the genome. Nevertheless, when these differences are not minor and become major differences, different implications may occur in the individual affecting his health and/or capabilities.

Germinal mutations are the ones and individual is born with. Somatic mutations happen during one's lifetime and are studied by looking at tumor cells and normal cells from the same patient at different times.

Figure 2.5 and Figure 2.6 show graphically how a mutation occurs within the DNA. This mutation can be of the form of a replacement, deletion or insertion of bases within the genome.

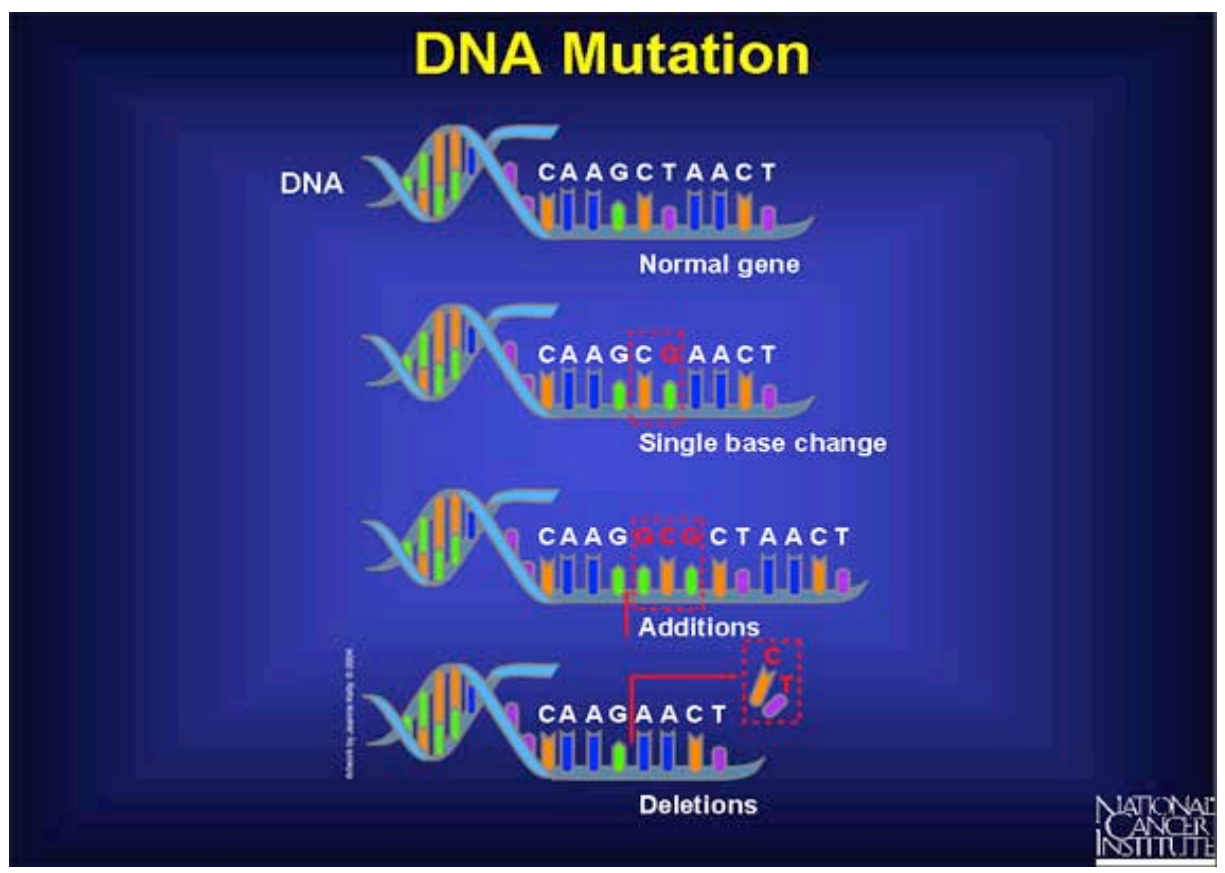

Figure 2.5. DNA Mutation. [23] 


\section{Types of Mutations}
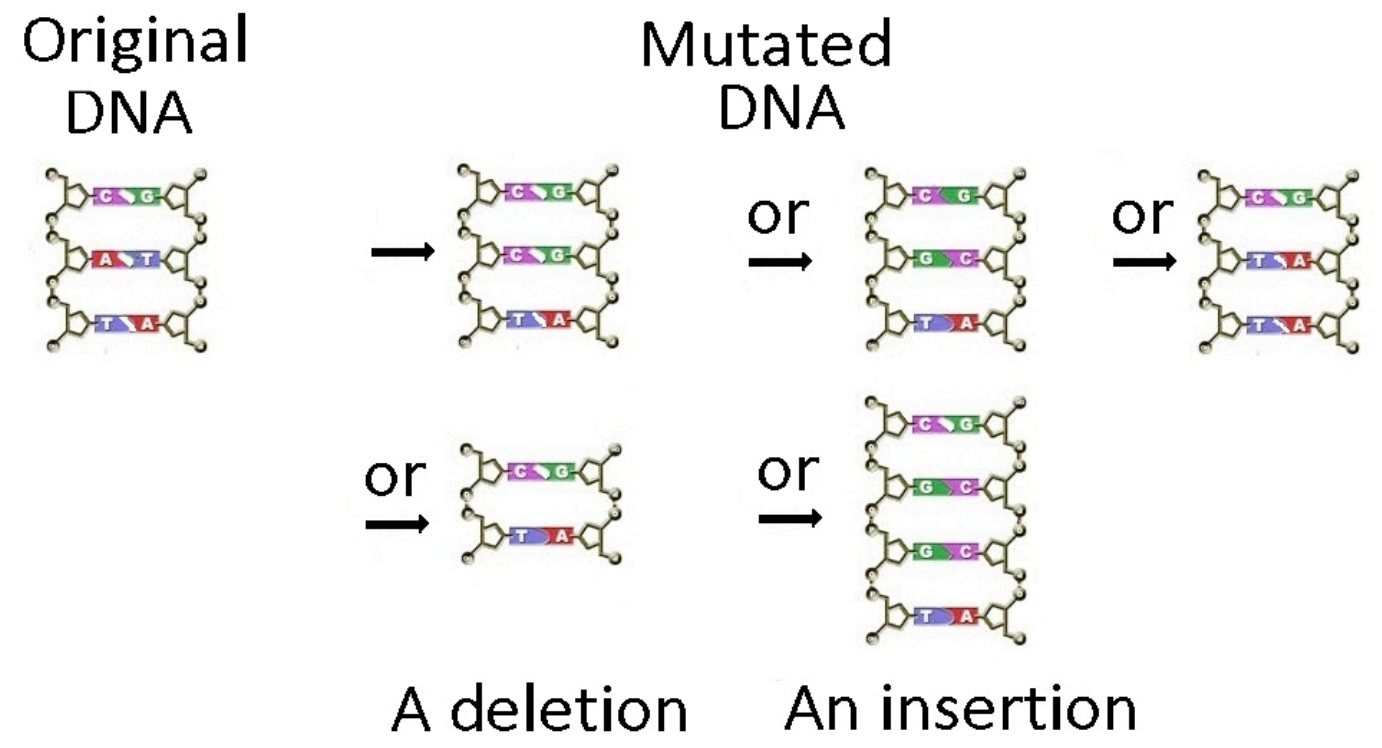

Figure 2.6. Types of DNA mutations. [24]

Many of these mutations are present since birth and many others are acquired during the development of a disease such as cancer.

These mutations have different forms and appear in different locations within the DNA. These mutations act as repeat sequences following a specific pattern or not. The two major types of repeat sequences are tandem repeats (TRs) including micro-satellites, mini-satellites, and satellites, and transposable elements (TEs), which are dispersed within genomes with a moderate to high degree of repetitiveness.

\section{i. Tandem Repeats (TRs)}

A common feature for both eukaryotic and prokaryotic genomes [25][26][27] is the 
repetition of both large and short sequences (2 to several hundred nucleotides long), known as tandem repeats. Many experts suggest that as much as $50 \%$ of the human genome is composed of repetitions [28].

An example of a tandem repeat is illustrated in Figure 2.7:

\section{ACTACTACTACT \\ Tandem repeat of 3 bases and length 4}

Figure 2.7. Tandem repeat example.

The biological role of repeated sequences has been researched by a number of scientists who have determined that they are linked to evolutionary mechanisms in prokaryotic organisms [29]. Tandem repeats [30][31] are common in eukariotyc organisms, as well. It has been reported that tandem repeats are responsible for over 30 inherited diseases in humans. Tri-nucleotide expansions have been associated with fragile $\mathrm{X}$ syndrome, myotonic dystrophy, Huntington's disease, various spino-cerebellar ataxias, Friederich's ataxia and others. Tandem repeats can exist in coding as well as in non-coding DNA. Expansion found in coding regions can affect the function of the genes involved by disrupting the normal synthesis of the proteins. When these repeats appear inside noncoding regions, it has been shown to affect gene expression via alterations in the transcription levels of the gene when the affected area correspond to either a promoter region, or a region containing factors that affect protein translation. Table 2.1 clearly illustrates genetic diseases related to Tandem Repeats.

Unfortunately, individuals suffering from inherited diseases do not have many choices in terms of treatment. However, in some cases an early diagnosis may help to ameliorate the devastating effects of the disease. Current tools available in clinics, as well as in 
bioinformatics allow the medical community to carry out diagnostics as early as in the pre-implantation embryo, to determine whether the embryo carry the expansions associated with a given disease or not.

Expansion of DNA repeats are not limited to inherited disorders only. Depending on the length of the repeat and the area it spans, tandem repeats are also known as "satellite, mini-satellite, and microsatellite" DNA. Blanes and Diaz-Cano [32], present a complementary analysis of microsatellite tumor profile in which they observe that changes in the number of repeats occur depending on the progression in the cancer cell, compared with the normal tissue. Multiple studies have shown micro- and mini-satellite instability during cancer progression in a wide variety of tumors, both familiar and sporadic. Breast and ovarian cancers show many more tandem duplications than other tumor types [33].

\section{Examples of genetic diseases caused by expanding trinucleotide repeats}

\begin{tabular}{llcc} 
& & \multicolumn{2}{c}{$\begin{array}{c}\text { Number of copies } \\
\text { of repeat }\end{array}$} \\
\cline { 3 - 4 } Disease & $\begin{array}{c}\text { Repeated } \\
\text { sequence }\end{array}$ & $\begin{array}{c}\text { Normal } \\
\text { range }\end{array}$ & $\begin{array}{c}\text { Disease } \\
\text { range }\end{array}$ \\
\hline Spinal and bulbar muscular atrophy & CAG & $11-33$ & $40-62$ \\
Fragile-X syndrome & CGG & $6-54$ & $50-1500$ \\
Jacobsen syndrome & CGG & 11 & $100-1000$ \\
Spinocerebellar ataxia (several types) & CAG & $4-44$ & $21-130$ \\
Autosomal dominant cerebellar ataxia & CAG & $7-19$ & $37-220$ \\
Myotonic dystrophy & CTC & $5-37$ & $44-3000$ \\
Huntington disease & CAC & $9-37$ & $37-121$ \\
Friedreich ataxia & CAA & $6-29$ & $200-900$ \\
Dentatorubral-pallidoluysian atrophy & CAC & $7-25$ & $49-75$ \\
Myoclonus epilepsy of the & CCCCCCCCCCC & $2-3$ & $12-13$ \\
Unverricht-Lundborg type & & & \\
\hline
\end{tabular}


Table 2.1. Examples of genetic diseases caused by expanding tri-nucleotide repeats. [34]

\section{ii. Transposable Elements (TEs)}

Transposable elements are mobile within the genome, and as such, they can be inserted anywhere, whether within the coding region of a gene, or within intronic regions. As a result of their insertion the expression of a gene is affected by changing the protein coded by the gene where insertion occurred, or by disrupting the natural context of the gene if the insertion occurred within an intronic region. The term "normal" context refers to the regions that surround the gene, meaning upstream and downstream regions that contain controlling elements, such as promoters, enhancers and inhibitors of gene expression. If the insertion occurred within any of those controlling elements, the gene expression would change. An example of a transposable element is illustrated in Figure 2.8.

\section{...ACTCCTTAAACTCGGTACTGGGGC... Transposable element: ACT}

Figure 2.8. Transposable element example.

We have to keep in mind that whether an insertion or modification of sub-sequence within a genome is considered a mutation event, whether one base or 300 bases, or more.

Table 2.1 shows the result of repeats affecting coding regions. Additionally, there are also reports of inherited and non-inherited diseases, including various types of cancer, associated with repeats in non-coding regions. Table 2.2 illustrates such scenarios as well and shows reports regarding transposable repeats, or transposable elements repeats related to genome mutations. 


\begin{tabular}{|c|c|c|c|c|c|c|c|c|}
\hline \multicolumn{9}{|c|}{ Pathologic Intronic Insertions in Humans } \\
\hline Retroelement & Length & Orientation & Position in Gene & Gene Name & Abbreviation & Phenotype & mRNA Studies & Reference \\
\hline \multicolumn{9}{|l|}{ Alu } \\
\hline AluYc1 & $316 \mathrm{bp}$ & antisense & $\begin{array}{l}-52 \mathrm{bp} \text { from the } \\
3^{\prime} \text { end of intron } 4\end{array}$ & glycerol kinase & GK & $\begin{array}{l}\text { benign isolated } \\
\text { glycerol kinase } \\
\text { deficiency }\end{array}$ & not reported & (Zhang et al., 2000) \\
\hline AluYb9 & $\sim 330 \mathrm{bp}$ & antisense & $\begin{array}{l}-19 \mathrm{bp} \text { from the } \\
3^{\prime} \text { end of intron } 18\end{array}$ & coagulation factor VIII & F8 & hemophilia A & exon 19 skipping & (Ganguly et al., 2003) \\
\hline AluYa5 & $331 \mathrm{bp}$ & antisense & $\begin{array}{l}-50 \mathrm{bp} \text { from the } \\
3^{\prime} \text { end of intron } 7\end{array}$ & $\begin{array}{l}\text { tumor necrosis factor } \\
\text { receptor superfamily, } \\
\text { member } 6\end{array}$ & FAS & $\begin{array}{l}\text { autoimmune } \\
\text { lymphoproliferative } \\
\text { syndrome }\end{array}$ & exon 8 skipping & (Tighe et al., 2002) \\
\hline AluYa5 & $368 \mathrm{bp}$ & antisense & $\begin{array}{l}-19 \mathrm{bp} \text { from the } \\
3^{\prime} \text { end of intron } 8\end{array}$ & $\begin{array}{l}\text { fibroblast growth factor } \\
\text { receptor } 2\end{array}$ & FGFR2 & Apert syndrome & $\begin{array}{l}\text { ectopic exon } 7 / 8 \\
\text { splicing in lieu of } 7 / 9\end{array}$ & (Oldridge et al., 1999) \\
\hline AluYa5 & $320 \mathrm{bp}$ & antisense & $\begin{array}{l}-44 \mathrm{bp} \text { from the } \\
3^{\prime} \text { end of intron } 5\end{array}$ & $\begin{array}{l}\text { neurofibromatosis } \\
\text { type } 1\end{array}$ & NF1 & $\begin{array}{l}\text { neurofibromatosis } \\
\text { type } 1\end{array}$ & exon 6 skipping & (Wallace et al., 1991) \\
\hline \multicolumn{9}{|l|}{$\underline{\mathrm{L} 1}$} \\
\hline L1(Ta) & $836 \mathrm{bp}$ & $\begin{array}{l}\text { sense and } \\
\text { rearranged }\end{array}$ & intron 5 & $\begin{array}{l}\text { cytochrome b- } 245 \text {, } \\
\beta \text { polypeptide }\end{array}$ & CYBB & $\begin{array}{l}\text { chronic granulomatous } \\
\text { disease (CGD) }\end{array}$ & $\begin{array}{l}\text { variable L1 } \\
\text { exonization; } \\
\text { exon } 5 \text { and } \\
\text { exon } 6 \text { skipping }\end{array}$ & (Meischl et al., 2000) \\
\hline L1(Ta) & $6 \mathrm{~kb}$ & antisense & $\begin{array}{l}\text { reported as } 3^{\prime} \\
\text { end of intron } 2\end{array}$ & hemoglobin, $\beta$ & $H B B$ & $\beta$-thalasemia & not reported & (Kimberland et al., 1999) \\
\hline L1(Ta) & $1.2 \mathrm{~kb}$ & sense & $\begin{array}{l}-24 \text { bp from the } \\
3^{\prime} \text { end of intron } 7\end{array}$ & fukutin & FKTN & $\begin{array}{l}\text { Fukuyama-type } \\
\text { congenital muscular } \\
\text { dystrophy }\end{array}$ & $\begin{array}{l}\text { variable exon } 7,8 \text {, } \\
\text { and } 9 \text { skipping }\end{array}$ & (Kondo-lida et al., 1999) \\
\hline L1(Ta) & $6 \mathrm{~kb}$ & sense & intron 1 & retinitis pigmentosa 2 & $R P 2$ & $\begin{array}{l}\mathrm{X} \text {-linked retinitis } \\
\text { pigmentosa }\end{array}$ & $\begin{array}{l}\text { no transcript } \\
\text { detected } \\
\text { by RT-PCR }\end{array}$ & (Schwahn et al., 1998) \\
\hline L1(Ta) & $2.8 \mathrm{~kb}$ & $\begin{array}{l}\text { antisense and } \\
\text { rearranged }\end{array}$ & $\begin{array}{l}-8 \text { bp from the } \\
3^{\prime} \text { end of intron } 3\end{array}$ & $\begin{array}{l}\text { ribosomal S6 } \\
\text { kinase } 2 \text { gene }\end{array}$ & RPS6KA3 & $\begin{array}{l}\text { Coffin-Lowry } \\
\text { syndrome }\end{array}$ & exon 4 skipping & (Martínez-Garay et al., 2003) \\
\hline \multicolumn{9}{|l|}{ SVA } \\
\hline SVA & $2.6 \mathrm{~kb}$ & sense & intron 1 & $\begin{array}{l}\text { low-density lipoprotein } \\
\text { receptor adaptor protein } 1\end{array}$ & LDLRAP1 & $\begin{array}{l}\text { Autosomal-recessive } \\
\text { hypercholesterolemia }\end{array}$ & $\begin{array}{l}\text { no expression } \\
\text { by northern blot }\end{array}$ & (Wilund et al., 2002) \\
\hline
\end{tabular}

Table 2.2. Pathologic intronic insertions in humans. [35] 


\subsubsection{Cancer Genomics Study}

The understanding of the genetic basis of disease in general, and of cancer in particular, has progressed dramatically in the past few decades. Several factors have been involved in that progress: some, purely of biological nature, and some at the intersection between biology and computational sciences. The science of genetics began in the 1860's when Gregor Mendel studied inheritance in pea plants (Pisum sativum) [36]. In the 1940's Oswald Avery, Colin MacLeod, and Maclyn McCarty showed DNA was the genetic material [37][38]. In 1953 James Watson and Francis Crick proposed the double-helix model for the structure of DNA [39]. The Human Genome Project (HGP) began in 1990 and was completed in 2003 by the International Human Genome Sequencing Consortium [40][41][42]. The reference genome produced by the HGP came from a single anonymous male donor from Buffalo, New York [43]. Since then, a number of international projects have started to elucidate the genomic sequence of various individuals. For instance, the HapMap Project which used DNA samples from 270 individuals, the 1000 Genomes Project, the Cancer Genome Atlas, the Cancer Genome Anatomy Project, and the Cancer Genome Characterization Initiative [36].

The Cancer Genome Atlas (TCGA) is a program supported by the National Institutes of Health (NIH). It will help to understand what turns a normal cell into a cancer cell. Its main approach as stated in its web site (cancergenome.nih.gov) is to compare DNA from normal and cancer tissue to find what are the differences. By utilizing such approach scientists working within the TCGA Project have learned that there are certain areas of the genome commonly affected in several types of cancers. Often these changes affect genes that control pathways in cells that cause cells to divide and survive when they 
normally would die. Another finding is that specific changes -also called signaturesallow scientists to tell one type of cancer from another. These signatures help doctors identify specific "types of cancer" which may respond differently to various treatments or have different prognosis. In order to better understand these concept I would like to define some of the specialized terminology (see cancergenome.nih.gov)

The genome is given by all the DNA that exists within a cell. For the majority of cells, the genome is wrapped into two sets of chromosomes inherited from both parents. These chromosomes consist of six billion DNA letters. The genetic alphabet consists of 4 letters: A, C, G and T. The area of Genomics consists of studying how these letters appear in a sequence and how every string of letters passes the information to the proteins, the real building blocks in the organism. DNA stands for deoxyribonucleic acid and instructions encoded in the string of DNA are passed to the proteins.

For cancer cells, changes in the bases (A,C,T, and G) can cause a modify the meaning of a genomic "word" or "sentence". These changes in the bases can make the cell to produce a protein that does not allow the cell to work as expected. These proteins could make the cell grow faster than normal and affect neighboring cells. The accumulation of these changes, or mutations, within a cell leads to the development of cancer. Mutations are classified as germ line and somatic. Germ line mutations affect germinal cells (oocytes or spermatocytes) and are transmitted to the offspring. Somatic mutations, on the contrary, affect somatic cells and are not transmitted to the offspring. The study of the genome in a cancer cell is known as cancer genomics. The field of cancer genomics has had a profound impact in the understanding of cancer progression at a molecular level. The field itself has been dramatically impacted by the application of the so called next- 
generation sequencing, or NGS, technologies, which has accelerated the pace of discovery, while reducing the cost. NGS technologies greatly depend on computer power to acquire, process, and analyze the data coming from sequencing the genomes of healthy, as well as cancer cells [44]. The importance of studies made at the intersection of cancer biology and computer sciences can be never be understated. As data generated from multiple sequencing projects grows, the computer capabilities should keep up with the challenges of analyzing the terabyte amounts of data. The strongest contribution of NGS on cancer genomics has been the ability to re-sequence, analyze and compare the matched tumor and normal genome of a single patient [44].

Finally, the sequence of the human genome and the discoveries brought about by it, or related to it, have allowed the faster integrations of genomics into the medical practice [45]. As cancer is defined as a genetic disorder due to the accumulation of mutations, tumor genome sequencing has been used to guide treatment in oncology, as well as to develop new therapeutic anti-cancer targets [45]. It is known, that cancer that look identical through the microscope may have very different underlying genetic changes and may respond differently to specific drugs. By identifying the mutational profile, or signatures, specific for a certain type of cancer, it would be possible to classify individual groups of patients who may have better responses to an specific treatment, and rule out patients that do not belong to such group, avoiding the secondary effects of an unnecessary therapy. 


\subsection{Related Work.}

We will analyze and compare our research from multiple perspectives since it involves work in different areas. We will start reviewing the different tools used for analyzing and comparing genomes and specific sequences and later we will do an extensive comparison of our research versus others which aimed at studying cancer and the genome relationships.

Numerous methods have been developed for analyzing the repeat structure of genomic sequences [46][47][48][49], most of which scan for a specific type of repeat such as short sequence repeats, palindromic repeats [50], tandem repeats [51][52][53][54], or highly periodic short repeat elements [55][56]. In most cases, such methods are unable to detect repeats that do not match a predefined pattern and intra or inter-genomic analyses are usually very difficult. Other methods [57][58], however, such as the use of Fourier transforms for repeat identification, do not search for a specific repeat pattern but rather try to locate occurrences of highly correlated periodic repeats. Nevertheless, this approach typically only identifies very strong genome-wide correlations such as those due to the triplet nature of the genetic code.

Among the tools we have in Bioinformatics, we identify the ones that look for sequences within another sequence or genome and the ones that look within established patterns (motifs).

\subsubsection{Fasta [59]}

First fast sequence searching algorithm for comparing a query sequence against a database. It is a tool that works for comparative analysis of DNA against DNA searches. 
One of its limitations is that it can only find one gapped region of similarity and performs relatively slow. However, on the positive side, it does not require specially prepared, preformatted databases.

\subsubsection{Blast [60][61]}

The Basic Local Alignment Search Tool (BLAST) was developed at the National Center for Biotechnology Information (NCBI) [62]. It is not normally used for DNA against DNA searches without translation because of optimization issues. This tool pre-filters repeat and "low complexity" sequence regions and it is capable of finding more than one region of gapped similarity. BLAST's implantation consists of a very fast heuristic and parallel algorithm with the restriction of precompiled, specially formatted databases.

\subsubsection{RepeatMasker [63][64]}

This program searches DNA sequences for repeats that present gaps between them. Sequence comparisons in RepeatMasker are handled by multiple search engines such as cross_match [65], ABBlast/WUBlast [66][67], RMBlast [68] and Decypher [69].

\subsubsection{Masker Aid [70]}

When RepeatMasker was created, scientists started to find repeat sequences for large genome sequences; however, they also found that it took fairly huge amount of time to the tune of hours to get the process completed on the fastest machines with high end processors and memory. MaskerAid was henceforth proposed to take the added benefit of WU-BLAST along with cross_match programs. As a result, this patch was applied to the 
RepeatMasker program in a 4 step process. The new proposed algorithm parses the outputs of BLAST programs and finds next the best alignments to find the repeats quickly. MaskerAid was run on all the existing conditions which cross_match satisfied and showed significant reduction in time taken across modes. Also the miss rate of repeat sequences was much reduced. The tool was also seen as lesser sensitive to userdefined/external parameters than RepeatMasker.

\subsubsection{Tandem repeat finder [71]}

Scientific studies show that tandem repeats have something special about them and they can be connected to important biological consequences observed in living beings. Hence, it is very important to find all Tandem repeats in DNA sequence quickly and efficiently regardless of the size of the genome sequence. Before the Tandem Repeat Finder appeared, the existing algorithms worked fairly well even though they had various disparities. Moreover, many had unacceptable worst case running times for large sequences. Few considered large and small sequences alike and to predict a pattern took unreasonable time for smaller sequences. Others were bounded by the inclusion of approximate repeats and due to the concept of substitution by a fixed number, it made little sense to apply the same algorithm to small and large sequence at the same time.

Tandem Repeat Finder takes all these factors into consideration and comes up with a solution which comprises of the following features and more: k-tuple matching and no size limit of repeats. This solution also finds hidden tandem repeats using a probabilistic model and, finally, aligns these repeats to obtain a consensus pattern. To end the process, these repeats are clustered together for further analysis. 


\subsubsection{Reputer [72]}

Exact repeat sequences are the ones that appear at least twice or more in the entire genome nucleotide sequence. Next, maximal repeats are basically exact repeats extended in both directions without loss of mismatch. These can be classified as Forward Repeats or Reverse Complement Repeats. A Reverse Complement of sequence AACCTTGG is CCAAGGTT.

Repeats are stored in the suffix tree for finding the pattern efficiently. Storage wise this algorithm is far superior to existing ones. In order to enumerate all repeats existing in the parsed sequence, a Depth-First search of the entire tree is required. This enlists one by one all the repeats with their coordinates and lengths stored in the end nodes. The algorithm is highly optimal from space and time considerations and can be further optimized for space constraints.

\subsubsection{The Vmatch large scale sequence analysis software [73]}

This work uses suffix arrays [74][75] to pre-process genomes, creating indexes which allow quick searching of probes. This brings the limitation given by the size of RAM memory present in the executing environment. At this time Vmatch in 32 bit computers has a limitation of genomes of 400 million bases.

\subsubsection{Amadeus [76]}

Amadeus is a software platform for genome-scale identification of known and novel motifs (recurring patterns) in DNA sequences, applicable to a wide range of motif discovery tasks. Amadeus can be used to identify binding site motifs from Protein 
Binding Microarray data. A dataset includes its measured binding intensities for each probe sequence covering together all possible 10-mers. In a number of competitions Amadeus ranked first (tied with one other group) in identifying the binding site motifs of $66 \mathrm{TF}$ datasets. Running time is a few seconds per dataset.

\subsubsection{Allegro [77]}

Allegro is a software tool for simultaneous discovery of cis-regulatory motifs and their associated expression profiles. Its inputs are DNA and genome-wide expression profiles. Its output is the set of motifs [78] found, and for each motif the set of genes it regulates (its transcriptional module). Allegro is highly efficient and can analyze expression profiles of thousands of genes, measured across dozens of experimental conditions, along with all regulatory sequences in the genome. Allegro has a user-friendly graphical user interface.

\subsubsection{Our work}

Our work works with mutations in general not necessarily following an exact or approximate match to any known signature. All mutations that we want to predict are made at the molecular level, and cover not only one specific gene nor chromosome but the whole human genome.

\subsubsection{Comparative chart}

Finally a comparative chart of our approach is presented together with other methods 
mentioned in related work in table 2.3. We can observe that our framework works on any type of sequences just like other applications; however, our work is oriented towards the prediction of new mutations over time in the human genome.

Table 2.3. Comparative chart of our work versus other approaches.

\begin{tabular}{|c|c|c|c|c|c|}
\hline Program & Alignment & Mismatch & $\begin{array}{c}\text { Exact } \\
\text { Match }\end{array}$ & Search against & Prediction \\
\hline Fafsta & YES & YES & YES & Any Sequence & NO \\
\hline BLAST & YES & YES & YES & Any Sequence & NO \\
\hline $\begin{array}{c}\text { Repeat } \\
\text { Masker }\end{array}$ & YES & YES & YES & Any Sequence & NO \\
\hline Masker Aid & YES & YES & YES & Any Sequence & NO \\
\hline $\begin{array}{c}\text { Tandem } \\
\text { Repeat } \\
\text { Finder }\end{array}$ & NO & YES & YES & Any Sequence & NO \\
\hline Reputer & NO & NO & YES & Any sequence & NO \\
\hline VMATCH & NO & NO & YES & Any sequence & NO \\
\hline Amadeus & NO & YES & YES & $\begin{array}{c}\text { Predetermined } \\
\text { database }\end{array}$ & NO \\
\hline Allegro & NO & YES & YES & $\begin{array}{c}\text { Predetermined } \\
\text { database }\end{array}$ & NO \\
\hline Our work & NO & YES & YES & Any Sequence & YES \\
\hline
\end{tabular}

\subsubsection{Other studies on cancer genomics}

One out of eight deaths is caused by Cancer [79]. A more in-depth understanding of cancer is a must in order provide better treatment for patients. Nowadays, genome data 
increases exponentially, which leads to more opportunities to learn insights of this terrible disease. David von Hansemann and Theodor Boveri examined strange chromosomal aberrations of cancer cells by dividing them under a microscope [80]. These findings indicated that cancer might be related to abnormalities at the chromosome level [81][82].

The molecular level analysis can be dated back to the 1980s, Reddy et al. [83] first associated a single base substitution of $\mathrm{G}>\mathrm{T}$ of the HRAS gene with the activation of that specific oncogene function in T24 human bladder carcinoma cells. That is the first study of identifying a certain mutation as being highly correlated to cancer. Currently, a generalizable concept of cancer states that malignancies result from accumulated mutations in genes that increase the "fitness" of a transformed cell over the cells surrounding it. The transformed cells sometimes acquire advantageous mutations which enable them to proliferate unlimitedly and sometimes allow them to spread to distant sites, leading to metastases.

\section{Application of Wavelets}

Wavelets transforms have become an integral part of mathematical analysis with an ever increasing range of applications, including genomic sequences. Prasad and Iyengar, 1997, in a publication titled Wavelet Analysis with Applications to Image Processing [84], describe several methods on the application of wavelets as a tool for feature extractions and other important methods in interdisciplinary applications. More recently, Meng, Chen, Iyengar and others [85], have published a paper on the applications of wavelets for 
cancer related genome sequences.

\section{Wavelet Analysis}

In order to conquer cancer, there is a high demand to delve into these sequences and mine useful information. From the signal processing point of view, biological sequences such as DNA sequence can be viewed as one dimensional signal. Accordingly, signal processing approaches can be adopted. The classic approach of Fourier transform suffers from the loss of temporal information. It does not give access to the signals' spectral variations during different time intervals. In other words, the time and frequency information cannot be seen at the same time, and thus a time-frequency representation of the signal is needed.

Wavelet analysis which is capable of decomposing time series into time-frequency space gets increasing amount of attention as a potential tool to study cancer genomic data. Figure 2.9 illustrates the general framework of the analysis procedure.

The origin of the wavelets theory dates back to the Fourier analysis developed by a French mathematician, Jean Baptiste Joseph Fourier (1768-1830) [86]. He came up with the idea of representing each signal as a weighted sum of cosine and sine functions, i.e., Fourier Trigonometric series. Most real-world signals' characteristics are non-stationary signals and vary in both time and space. FT is trying to capture frequency content. The calculation for FT is in Equation (1) from [84]. 


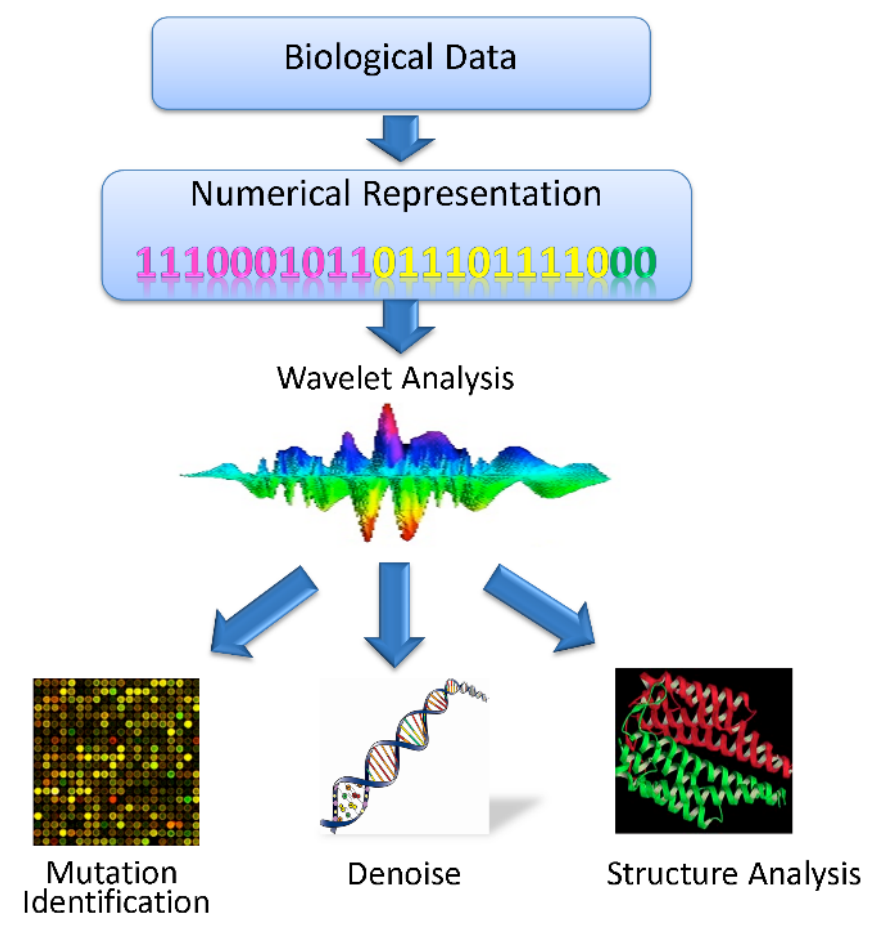

Figure 2.9. Sample procedure of applying wavelet transform in cancer genome analysis.

The FT defines the global representation of the frequency content of a signal over a total period of time. However, it does not represent to the signal's spectral variations during this interval of time. In other words, people cannot view the time and frequency information concurrently. Nevertheless, it would be helpful if both pieces of information were available.

To achieve this, Dennis Gabor proposed the Short-Time Fourier Transform (STFT) to study a small fraction of the signal at a time by segmenting the signal using windows [87]. This obtains the specific contents of each of the analyzed sections separately. The segment of signals in each section is assumed stationary. 
However, an important question is raised, which is how to determine the window size. As is shown in experiments, a narrow window results in a poor frequency resolution, whereas a wide window leads to poor time resolution. In addition, one cannot determine the time intervals where a certain frequency exists. Therefore, the wavelet transform was initially proposed as an alternative approach to STFT to overcome the resolution problem. All other windows are the dilated, compressed, and shifted versions based on the mother wavelet.

In terms of history, Haar Wavelets family was developed by Alfred Haar in 1909 [88]. The simplicity of this wavelet transform gives it broader applications. It is generally used to analyze a given signal in terms of functions which are more finite in time than the harmonic functions used in the Fourier analysis. In 1980s, Jean Morlet replaced the Gabor window used in Short-Time Fourier Transform (STFT) [89] by stretching and compressing oscillating windows. Using this technique, he got more reliable and accurate results. This approach is later named as the Morlet Wavelets. In 1989, the idea of multiresolution was proposed [90]. This idea forms the base theory of versatile wavelets families. Based on this concept, the well-known and frequently used Daubechies wavelets family was invented [91].

To sum up, wavelet analysis techniques have the following advantages over the traditional FT [92]: (a) wavelets are capable of analyzing both stationary and nonstationary signals, while FT gives less information in analyzing non-stationary signals; (b) wavelets have a god localization of frequency and time domains while the standard FT is only hgandled in frequency domain; (c) the base functions of wavelets can both be scaled and shifted and therefore are more flexible, while the FT can only be scaled; and 
(d) wavelets have a much wider range of applications than FT. For example, they could be used in non-linear regression and compression. A brief summary of the comparison is shown in Table 2.4 .

\begin{tabular}{l|l|l|}
\hline Properties & Fourier & Wavelet \\
\hline Stationary signal & Yes & Yes \\
\hline Non-stationary signalNo & Yes \\
\hline Time domain & No & Yes \\
\hline Frequency domain & Yes & Yes \\
\hline Scale & Yes & Yes \\
\hline Shift & No & Yes \\
\hline
\end{tabular}

Table 2.4. Comparison of Wavelet Analysis and Fourier transform (FT). [85]

Renato Dulbecco raised the point that the complete sequence of the human genome would be an essential tool for systematically discovering the genes that cause cancer [93]. The recent advance of next-generation sequencing technology makes the whole cell genome analysis possible for individual cancer case. In recent years, data about epigenome and transcriptome on a genome-wide scale of cancer grow exponentially [94], which offers great opportunity to unlock the mystery of cancer. The main streams of existing work of applying wavelet analysis in cancer genome research are summarized as follows.

Cancer is caused by mutations, Generally speaking, there are four types of common mutations in the cancer genome, which are substitution, insertion or deletion (indel), copy number altercations, and translocations. They are illustrated in Figure 2.10. 


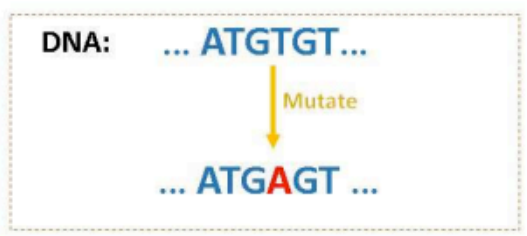

Type 1: Substitution

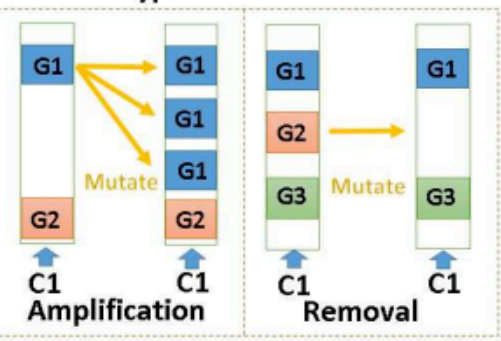

Type 3: Copy Number Alteration

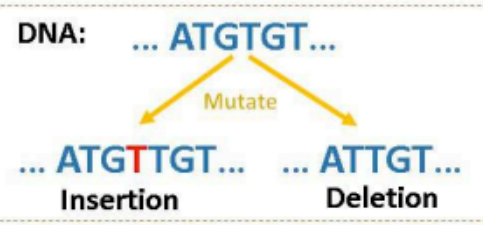

Type 2: Insertion/Deletion

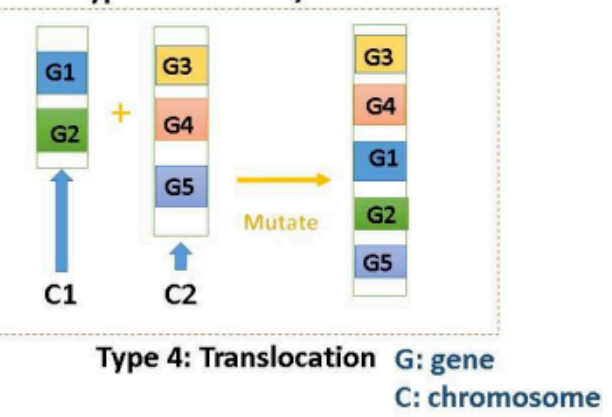

Figure 2.10. The four types of mutations. [92]

\section{Wavelet Analysis in Insertion/Deletion Mutations in Cancer Genome}

As described in previous section, cancer is caused by different mutations in the cancer genome. Recent studies find that the form and rate of mutations depend on the context of the mutation point [95]. Wavelet analysis finds its application in this scenario since it provides a multi-scale analysis on the sequence without pre-defined prioris. Therefore, it is suitable for detecting spatial patterns of the sequences around the mutation point without any prior knowledge. In a previous study [96], the authors targeted at identifying the spatial distributions of seven types of mutation related motifs, such as deletion hotspots, DNA pol pause/frameshift hotspots, etc, with respect to insertion/deletion break points. The authors first computed the motif frequency to generate the mo- tif frequency profile. Because of the computational simplicity, Haar wavelet analysis was applied to de- compose the frequency profile. The coefficients' sec- ond raw moments on a multi- 
scale basis were computed and they were used to measure the size of the difference between motifs occurrence patterns in insertion/deletion flanks vs. control regions. Their study identified the significant spatial distribution patterns of mutation motifs. The identified motifs could be utilized as targets for some cancer medicine. In another study presented in [97], the authors collected 1625 spontaneous base-pair substitutions in the MutL2 strain of Escherichiacoli and analyzed the spatial distribution of these mutations across the Ecoli genome. In order to accommodate the total number of mutations and describe the data clearly, the researchers generated 46 bins, each of which contains $100 \mathrm{~kb}$ nucleotides, starting at the origin of replication. A histogram was generated to show the distribution of missense mutations. Next, the Fourth- order Daubechies wavelet transforms were applied since it is able to remove jumpy appearance of the Haar averaged signals. The analysis found that these mutations are not distributed at random but, instead, fall into a wave-like spatial pattern that is repeated almost exactly in a mirror image in the two separately replicated halves of the bacterial chromosome. These findings give some insight on different mutations occurred in cancer genome.

\section{Wavelet Analysis in Copy Number Alterations}

Copy number alterations are a common pattern of structure variations in cancer genome. This sort of mutations is one hot research area in cancer genome research nowadays. Generally speaking, the main methods of detecting this sort of mutations, fall into two categories, which are the microarray-based gene expression analysis approach and the next generation sequencing-based approach. There have been studies utilizing wavelet analysis in both approaches. In terms of the first approach, the study [98] is an exemplar. 
In this paper, the expression level values of each gene are on the vertical dimension and locations of genes on the chromosome are the on the horizontal dimension. The problem of detecting copy number variations was modeled as extracting salient information on the transformed curve, such as sharp peaks and drops of the signal under the high noisy situation. As mentioned previously, although Fourier transform is helpful in signal processing to transform the time series to frequency domain, it loses information regarding position of signal changes, which is important information gain from this study. Therefore, wavelet transforms which capture information in both the frequency and time domains, are suitable for discovering sharp discontinuities on the genome locations. Specifically, the signal profile is first decomposed into a family of multi-resolution subbands using Haar wavelet. For each subband, p-values are assigned to Haar coefficients based on a null-distribution estimated from normal reference samples. Following that, significant coefficients are selected by assigning thresholds for false discovery rate. The selected coefficients are used to identify copy number alterations. The reason to select the Haar wavelet transform is that it is good for analyzing piecewise constant copy number signals [99]. Other similar studies following this direction include [100] and [101].

The next generation sequencing-based methods provide a novel and effective solution for analyzing the copy number alterations in a relatively high resolution. Nevertheless, it suffers from the relatively high noise issue [102]. One benefit that the wavelet analysis provides is its capability of decomposing the signal into a spectrum of different frequencies, where many high frequency components are corresponding to noise. Therefore, one direct application of the wavelet decomposition is to do the noise 
reduction. In [103], a CNAseg algorithm is proposed to identify copy number alterations from the second-generation sequencing data. In this study, the counts of copy number along the chromosome coordinates were converted to the discrete signals and an undecimated discrete wavelet transform was utilized to smooth the counting data. The number of decomposition levels was adapted to the length of the window counts for each chromosome. The reconstructed signal then went through the Hidden Markov Model (HMM) for segmentation and the Chi-square statistics based segment merging step. Experimental results demonstrated that those proposed approaches decrease the number of non-copy number alteration induced HMM segments and reduce the unevenness in read depth. Such reduction helps improve the performance of the system. The wavelet decomposition-based noise reduction is commonly used in studies in this research direction, such as in [100] and [102].

\section{Wavelet in Machine Learning Research Frame- work of Cancer Genome}

Nowadays, cancer genomics and proteomics data increase faster and faster. Meanwhile, more and more machine learning based approaches are applied by researchers for cancer genome analysis. We assume here that mathematical models could be built to learn patterns and could make predictions for unseen data by mining the patterns from the existing data. Firstly, the raw data are to be converted into a related compact and meaningful representations for this propose. We usually call this process as feature extraction. It could be utilized to extract features from a series because wavelet analysis could capture the global and local characteristics of sequence data. My applications used wavelet analysis as feature extraction approach. As an example, in [104], researches 
utilize wavelet analysis to extract features from DNA microarray data in order to extract important features for classification. Another example is that Liu et al. [105] proposed a framework which utilizes wavelet to extract features from thousands of protein markers in survival analysis in the field of colorectal cancer. Daubechies wavelet $\mathrm{db} 7$ is utilized by Liu to perform the continuous wavelet transform to extract the coefficients from the protein marker expression data. These coefficients contain information at different scales of the original biomarker signal. Afterwards, they are been used as features for cancer classification.

Cancer is a very terrible disease which caused by mutations and one of the most problem human faced in this generation. It is viewed as a genetic disease. In conclusion, a good understanding of the mechanism of mutations is mandatory to combating this disease. Wavelet analysis has found its application in many areas of cancer genome research, such as mutation identifications and cancer bio-marker identifications. It can perform multiscale analysis and capture the local and global information of a time series.

Similar to the Wavelet technique previously discussed, our work also utilizes a machinelearning approach in the analysis of the cancer genome. Specifically, speaking, our objective of using such approach is being able to make predictions of mutations within the genome of a patient.

\subsubsection{Clinical perspective}

From the medical point of view, our analysis focuses mainly on cancer, a disease that is caused by mutations and affects the genome leading to instability and the generation of more mutations. Through numerous studies, scientists have been able to relate certain 
cancers somatic mutations in different genes in the genome. As a result, classifying cancer based on gene expression has provided much insight into the complex landscape of multiple interactions between gene networks, as well as into possible treatment strategies. Furthermore, the constant appearance of more sophisticated sequencing strategies have helped in the advance of the study in cancer genomics. This has allowed scientists to interrogate cancer-specific genomic variants and compare them with the normal variants in the same patient. Next generation sequencing (NGS) technologies can provide genome-wide coverage at a single nucleotide resolution and at reasonable speed and cost. By having the unprecedented molecular characterization provided by NGS offers the potential for an individualized approach to treatment. These strategies aim to provide the definition of relatively characteristic gene expression profiles, or molecular signatures that may have prognostic implications for targeted therapies.

Consequently, in cancer patients, the objective of NGS is to obtain and compare information about cancer and normal tissue DNAs. By analyzing this, scientists can develop a catalog of somatic variants that exist in tumor genome but not in the normal tissue DNA. The objective of the analysis is to reveal a drug target in the examined cancer which facilitates the selection of therapy, and improves the personalized risk assessment. As a result, determining the molecular signatures of genes mutated in cancer may help to predict the clinical outcome.

Researchers and clinicians can benefit from being able to predict molecular signatures based on the initial events in cancer development in order to, develop therapeutic modifications in treating the patients. Nevertheless, predicting such signatures at the time of the tumor diagnosis is a major challenge. Numerous research groups have reported 
lists of predictive genes and reported good predictive performance in terms of prognosis and potential for malignancy based on them. However, the gene lists differed widely and had only very few genes in common.

Additionally, those types of prediction were based on computational approaches not involving NGS, such as microarray analysis, qPCR, and others in various types of cancer such as colorectal, lung, prostate, breast cancer and others.

After searching PubMed we found very few articles reporting predictions in cancer gene signatures by utilizing the NGS approach [106][107]. Furthermore, these reports have dealt with predictions from data obtained at one point in time from paired normal and cancer tissues. To the best of our knowledge, there have been no reports on predictions of cancer gene signatures during cancer progression in the same individual.

Thus, the disadvantages of current predictive models in cancer are that they are focused on evaluating mutations that anticipate the risk of progression and its clinical impact on the length of patient's survival. They are not intended to predict mutational events at molecular level, only to detect and classify existing mutations. Thus, they are not suitable for making predictions in terms of the molecular behavior of the cancer genome.

In our hypothesis, by having the full sequence of the genome from both normal and cancer tissue at different points in time, we are able to extract valuable information to validate the predictions generated from genome data before relapse time. After an exhaustive search of the available scientific resources (PubMed, Google Scholar), we could not find reports utilizing such strategies.

In order to fill this gap, in our study, we proposed to search for studies of cancer patients with such identified mutated genes by the use of NGS various points in time. The 
mutation profile identified earlier in time will serve as the basis for the prediction at later in time. Furthermore, in order to evaluate our prediction results, we compared the observed mutated locations at the time of relapse with the results of our own predictions.

Our main objective in this study was to test a computational framework utilizing published data from a longitudinal study of patients with acute myeloid leukemia (AML) whose DNA from both normal as well as cancer tissues were subjected to next generation sequencing analysis at various points in time. First of all, we processed the sequencing data at the time of cancer discovery. Secondly, we tested our framework by predicting the regions of the genome to mutate at the time of relapse. Finally, we compared our results with the observed mutated regions, identified by sequencing their genomes at the time of relapse and determined that our predictions are in good agreement with the reported data. 


\section{CHAPTER 3}

\section{RESEARCH PROBLEM AND SPECIFIC AIMS}

In this chapter we motivate the problem to be investigated and present a detailed problem statement. Our research focus is in the area of Bioinformatics. In the next section we provide the motivation for this research by emphasizing the need for a prediction framework for longitudinal DNA mutations within the human genome and highlighting the benefits to be gained from conducting the study. In Section 3.2 we concisely describe the problem to be investigated.

\subsection{Motivation}

Over time, the scientific and industry community have made tremendous efforts on developing computational solutions to aid the research in molecular biology. These computational solutions focus mainly on String algorithms since the DNA can be represented as a sequence of a combination of four characters (ACTG). These characters are basically what we call the bases in the DNA. Nevertheless, when these sequences suffer from alterations in their structures (changes in one or more bases at different locations within the whole sequence), implications in the health of the individual may appear. Many diseases are associated with causing these changes (mutations) within the genome of an individual. In our case, we are concerned specifically with cancer.

In achieving this goal, we built a computational framework that will perform in three main stages. Firstly, the framework will pre-process the files containing the patient's data for feeding the information needed by the prediction stage. Secondly, given the information provided by the previous stage, the framework will perform the prediction of the number of mutations to occur as well as the locations where these will happen through a statistical analysis. Finally, after the framework finishes with the predictions, 
the predicted number of mutations as well as their locations will be presented in more than one format to the physicians to provide treatment and/or make further discoveries as many predicted locations could occur at areas where no known functionality exists. Additionally our framework has been implemented in Java and also utilizes statistical libraries and tools for accuracy and performance. Thus, our software follows the Object Oriented (OO) paradigm and has been developed on the Eclipse Integrated Software Environment (IDE) with the currently latest Java Development Kit (JDK 7).

\subsection{Problem definition}

These changes or mutations in the genome can cause various disorders in the individual depending on their number and locations. As a result, knowing how many mutations will occur as well as where they will occur become very important matters.

The effort is divided into the following three sub-problems:

1. Being able to perform statistical analysis on a given patient's genomic data.

2. Making reliable predictions for the mutating behavior of the given sequence.

3. Identifying mutations in regions in the genome that have not been considered functionally important or relevant.

In order to target the solution of these problems we target three specific aims. Each of these provides a solution to every sub-problem previously respectively. 


\subsection{Specific Aims}

\subsubsection{Aim \# 1: To build a tool for statistical analysis on genomic data sets.}

In order to carry out our first contribution, we built a framework which is capable of extracting statistical information from the genomic data from a patient. It is very important to mention that our framework works with the whole genome data from a patient and not just with one chromosome or a couple of them. Thus, given the genomic data from a patient, we proceed with an analysis at the chromosome level of the whole genome. This analysis involves producing statistical data from the mutations present in each chromosome. The input for this first stage is the genomic data from a sick patient who already presents mutations in his genome.

The statistical analysis involves building a series of variables based on the actual genomic data from the patient that later will be used to perform the predictions.

\subsubsection{Aim \# 2: To utilize this data set and formulate a prediction model.}

Our second goal is our primary contribution as it deals with the prediction itself. Our prediction works at two levels. First, we will predict the number of mutations to occur within one chromosome and, second, the locations where these mutations will happen. For this contribution, our framework will make usage of the data obtained at the completion from the previous stage to build a prediction model to determine the number of mutations to occur. Later, the framework will use another algorithm (Section 7, Fig. 7.4) to predict the respective locations for these mutations.

\subsubsection{Aim \# 3: To facilitate the discovery of new functionalities in areas of the genome that had not been previously implicated in cancer.}

Finally, our third goal consists on providing information to the physicians and scientists 
that would lead to the discovery or redefinition of new genes and or functionality of certain areas of the genome. This happens because when determining the locations of the newly predicted locations, scientists will be able to find out that some of the areas in the genome where these mutations are predicted to appear do not belong to any known gene or known functionality but that they still have repercussions on the patient's health and, as a result, it can be the case that a new gene can be identified or redefined (e.g. the range of the gene should be extended) or that perhaps a new functionality from this area has been discovered. This information can be provided to the experts in the area of genetics since they can perform further analysis in the laboratory to determine what is there or what functionality might be hidden. Additionally, a specialized and personalized treatment will be possible to obtain due to the fact that we genomic data used was specific to the patient.

The importance of our work resides on being able to predict mutations in a patient's genome ahead of time in order to provide information that will potentially assist physicians on the selection of personalized therapies they can give and/or the development of new specialized drugs.

This prediction is done at the gene and chromosome levels. It provides physicians with information to make a reliable estimation of the effects of a mutation. Moreover, it may lead to the discovery of unknown functionality within the human genome 


\subsection{Significance and impact of our work}

Cancer progression is a complex, multistep process, leading to genomic instability and loss of growth control. Scientists are trying to identify the so-called driver mutations and differentiate them from the "passenger" mutations that passively follow the driver mutations. Without knowledge of the biological functions of the genes this process is very difficult to accomplish. Furthermore, a great deal of genes has no known function, which make it almost impossible to analyze by this approach.

Based on the mutational profile of the cancer cells before relapse time, we utilized the framework to make predictions of new mutations at a later time. The most interesting aspect of this work is the possibility of comparing the predictions with real experimental data obtained from the same patients at the time of cancer relapse. Those comparisons allowed us to generate accuracy values (between $80 \%$ and $92 \%$ accuracy) which are remarkable. To the best of our knowledge, there are no reported studies directed toward the prediction of changes at molecular level. Numerous studies dealing with predictions based on the mutational signatures in cancer cells are limited to the predictions in clinical outcomes, rather than in the changes at the genome level in the cells of a patient.

One of the contributions of our research is the possibility of validating the predicted mutational profile with the experimental data. This situation has been possible as a result of the revolutionary advances in genome sequencing and data analysis. The second contribution of our research is the purely computational approach to make predictions with no need to include data on the underlying biological processes. This is by far the most exciting possibility. Scientist working on the molecular biology and genetics of cancer experience great difficulty in characterizing the function of an unknown gene which is the first step toward its identification whether is critical or not in cancer progression. 
By utilizing our approach, there are no requirements to ascertain the function of genes for the prediction to take place. The third contribution is about providing a target for cancer therapy. As a result of the prediction, scientist will obtain putative target genes to identify, characterize, and possibly utilize as targets of pharmacological agents for cancer treatment. 


\section{CHAPTER 4}

\section{DATA MINING AND STATISTICAL ANALYSIS}

\subsection{Background}

Our framework is implemented in Java and uses internal and external libraries. The external library we employed for the statistical analysis and processing of our data is Weka.

After our framework processes the input genomic data and obtains the initial statistical information for the DNA mutations for the patients at the chromosome level (explained in further detail in Chapter 6), Weka is employed for creating a model at the chromosome level. This external library builds a model to predict the number of mutations to occur per chromosome.

We chose Weka since it is one of the most widely adopted tools in academia for the purposes of machine-learning and statistical analysis.

\subsection{Weka [108]}

Weka is acronym name that means Waikato Environment for Knowledge Analysis. It is a software library and tool that consists of various machine-learning algorithms written in Java.. Weka complies under the GNU General Public License. Weka provides features for data mining targeting classification, data preprocessing, clustering, regression, , among others. Essentially, the purpose is to have an application that can be trained for machine learning capabilities and obtain meaningful information through trends and 
patterns. Weka is open source and is written in Java. Consequently, it is cross-platform. Weka brings a user-friendly and graphical interface that allows for quick set up and operation as well as a programmable API. The data we use to feed Weka is given in the form of as a flat file or relation, meaning that every data object has to be described by a specific number of attributes of a specific type which can be either alpha-numeric or numeric values. In essence, this tool allows users to discover information that is normally hidden from database and files in general. Classification is the core of Weka's functionality. All of the newest and older machine-learning (ML) algorithms follow an object-oriented (OO) Java class hierarchy. Additionally, regression, association rules and clustering algorithms are also part of Weka's implementation.

All the following definitions and examples regarding Weka were obtained from [109].

\section{Weka basic concepts}

For a better understanding of Weka, certain concepts are to be clarified.

\subsubsection{Dataset}

A dataset is an essential concept for machine-learning and it can easily be thought of as a two-dimensional array or table. A dataset represents a collection of samples which are of class Instance. Attributes from an instance are nominal, numeric or strings. Externally speaking, the representation of an Instance class is a file in ARFF format. This file contains a header with the attribute types and the data as comma-separated list. 


\subsubsection{Classifier}

Every learning algorithm is a sub-class from the abstract Classifier [110]. The requirements for a classifier are: a routine for the classifier model from a training dataset and another one for evaluating the generated model.

\subsubsection{Weka from Java - API}

WEKA functionality can be called from Java sub-routines by the utilization of its API. It runs similar to when calling classifiers with $-p 0$. Nevertheless, unlike Evaluation, it produces the full class probability for any classifier. The java API comes in the form of a jar file and can be incorporated into any Java project. The functionality can be invoked at any time from any pat from the source code of the program.

\subsection{Statistical Analysis}

Numerous statistical analysis have been developed through time in order to model complex systems or problems [111][112][113][114]. Iyengar and Rao present a great analysis on statistical techniques for modeling complex systems [115]. Here they present single and multi-response models that are representative of modeling complex systems. Their work starts with various concepts related to linear and nonlinear models and then examines four representative techniques of model discrimination that utilize non-intrinsic and intrinsic parameters, Bayesian methods, and likelihood discrimination. Additionally, the authors also evaluate multi-response models which deal with issues from design of experiments for parameter estimation and model discrimination.

Similarly, as mentioned in the previous section, Weka allows for a selection of a variety 
of models and machine-learning algorithms. Depending on the size and type data we are dealing with, one model will adjust better for prediction analysis than another. Thus, in our scenario, linear regression was the model that best fit to our predicting and gave more accurate results. However, we are open to the inclusion of other models in the future as our data size increases and therefore, the usage of the Weka API in our framework in order to achieve future adaptability and scalability.

\section{Linear Regression}

The approach of Regression is applied in statistics to model a relationship between one dependent variable and a set of one or more explanatory/independent variables [116]. If the explanatory variable were just one, then we call it simple linear regression. In any other case, it is called multiple linear regression.

Linear regression is used mainly in two types of applications:

1. For prediction. Here we build a predictive model for a given set of observed data. With this model, we can perform predictions for a future point in time based on a set of previous points in time (training data).

2. For measuring the intensity of the relationship among the dependent variable and the explanatory variables. This allows to know which explanatory variables have higher impact on the value of the dependent one and which ones don't.

The least squares approach is the most widely used method to calculate the coefficients from a linear regression model. 
The model has the following form:

$$
y_{i}=\beta_{1} x_{i 1}+\cdots+\beta_{p} x_{i p}+\varepsilon_{i}=\mathbf{x}_{i}^{\mathrm{T}} \boldsymbol{\beta}+\varepsilon_{i}, \quad i=1, \ldots, n,
$$

where all betas are the coefficients and all the $\mathrm{Xi}$ are the features. Finally, epsilon is the error or noise to adjust the model. 


\section{CHAPTER 5}

\section{MODELING CANCER RELATED GENOME SEQUENCES OF PATIENT DATA}

This chapter is directly related to our second contribution which is the creation of a model to predict the DNA mutations. Here, we discuss the reasoning behind the selection of our model, that is, the linear regression model.

\subsection{Introduction}

The importance of modeling of cancer related genomic data has generated much attention from researchers from various interests, primarily from pharmaceutical design companies. The most common means of finding correlation of genomic data is by structuring a statistical model for the system through which we can predict, control and optimize the variables. A detailed exposition of statistical techniques in modeling of complex systems (single and multi-response models) can be seen in a well-known publication [115]. This chapter examines a representative technique for designing experiments and providing a mathematical structure for the analysis of the data that we have collected in our study.

\subsection{Statistical analysis for our model}

In this study, the prediction values are numerical. From the perspective of data mining, this problem can be modeled as a regression problem. In our research problem, one challenge is that the training data instances are limited since it is costly to gain patient 
data. As is known in data mining field, fitting a high order model would require learning more parameters, which needs a large number of samples.

If we tried to use a higher order model such as non-linear models, then we would be facing the problem of over fitting. The results in such case would be totally off as we could observe when switching Weka to other algorithms. When using a higher order model, the number of instances has to be considerably larger compared to the number of features in the model; however, in our case, the number of features and the number of instances have a ratio of almost 1 to 1 which obviously would bring the problem of over fitting.

Given this observation, the linear regression model which fits a linear equation to features and the target variable is utilized. Specifically, three selected numerical features (explained in Section 7.3) are normalized and fit to the linear regression model. The general form of the model is represented as $Y=\boldsymbol{a}+\boldsymbol{b} \boldsymbol{X}$, where $Y$ is the predicted value and $\boldsymbol{X}$ is the normalized feature vector. $\boldsymbol{b}$ is the weights and $a$ is the error term. In addition, our prediction task has a temporal dimension. Assuming there are $N$ observation time points, which are $T_{1}, T_{2}, \ldots, T_{N}$, suppose we want to predict the mutation number at time point $T_{N}$ utilizing the features from $T_{1}$ to $T_{N-1}$. A natural assumption is that the mutation number is highly correlated with features at time point which are close to $T_{N}$. However, it is very difficult to measure this numerically. The linear regression model provides a nice way of quantifying the contribution of each feature. The significance of the features are represented by the corresponding weights trained in the model under the condition that all features are normalized. For example, if the learned parameter for feature $F 1$ is 100 , and the other parameter for feature $F 2$ is 0.01 . We can conclude that 
F1 is more important. This property can give us more insight in our study as we want to know which features are important.

\subsection{Mechanisms and Models}

In order to fit the linear regression model, the mathematical approach of learning the parameters needs to be defined. In this dissertation, we model the learning of parameters as an optimization problem, in which the object function is the mean square error. Formally, assume for the predicted value $y^{i}$ for data instance $i(1 \leq i \leq M), M$ is the total number of chromosomes in one patient. Three features are represented using $F_{1}^{i}, F_{2}^{i}$, and $F_{3}^{i}$. Let the corresponding parameters be $\beta_{0}, \beta_{1}, \beta_{2}, \beta_{3}$. The cost function, which corresponds to the half mean square error can be summarized as follows:

$$
J=\frac{1}{2 M} \sum_{i=1}^{M}\left[y^{i}-\left(\beta_{0}+F_{1}^{i} \beta_{1}+F_{2}^{i} \beta_{2}+F_{3}^{i} \beta_{3}\right)\right]^{2}
$$

In order to minimize this function, the gradient descent algorithm can be utilized. The partial derivative of the function with respect to $\beta_{0}, \beta_{1}, \beta_{2}, \beta_{3}$ are as follows:

$$
\frac{\partial J}{\partial \beta_{0}}=\frac{1}{M} \sum_{i=1}^{M}\left[y^{i}-\left(\beta_{0}+F_{1}^{i} \beta_{1}+F_{2}^{i} \beta_{2}+F_{3}^{i} \beta_{3}\right)\right]
$$




$$
\begin{gathered}
\frac{\partial J}{\partial \beta_{1}}=\frac{1}{M} \sum_{i=1}^{M} F_{1}^{i}\left[y^{i}-\left(\beta_{0}+F_{1}^{i} \beta_{1}+F_{2}^{i} \beta_{2}+F_{3}^{i} \beta_{3}\right)\right] \\
\frac{\partial J}{\partial \beta_{2}}=\frac{1}{M} \sum_{i=1}^{M} F_{2}^{i}\left[y^{i}-\left(\beta_{0}+F_{1}^{i} \beta_{1}+F_{2}^{i} \beta_{2}+F_{3}^{i} \beta_{3}\right)\right] \\
\frac{\partial J}{\partial \beta_{3}}=\frac{1}{M} \sum_{i=1}^{M} F_{3}^{i}\left[y^{i}-\left(\beta_{0}+F_{1}^{i} \beta_{1}+F_{2}^{i} \beta_{2}+F_{3}^{i} \beta_{3}\right)\right]
\end{gathered}
$$

In each iteration of updating the parameters, $\beta_{0}, \beta_{1}, \beta_{2}, \beta_{3}$ are updated as follows:

$$
\begin{aligned}
& \beta_{0} \leftarrow \beta_{0}-\alpha \frac{\partial J}{\partial \beta_{0}} \\
& \beta_{1} \leftarrow \beta_{1}-\alpha \frac{\partial J}{\partial \beta_{1}} \\
& \beta_{2} \leftarrow \beta_{2}-\alpha \frac{\partial J}{\partial \beta_{2}} \\
& \beta_{3} \leftarrow \beta_{3}-\alpha \frac{\partial J}{\partial \beta_{3}}
\end{aligned}
$$

Using the matrix annotation, assuming matrix $\boldsymbol{F}$, which is shown as follows:

$$
\boldsymbol{F}=\left[\begin{array}{cccc}
1 & F_{1}^{1} & F_{2}^{1} & F_{3}^{1} \\
\cdots & \cdots & \cdots & \cdots \\
1 & F_{1}^{i} & F_{2}^{i} & F_{3}^{i} \\
\cdots & \cdots & \cdots & \cdots \\
1 & F_{1}^{M} & F_{2}^{M} & F_{3}^{M}
\end{array}\right]
$$


And the parameter $\boldsymbol{\beta}$ and the response vector $\boldsymbol{y}$, which is defined as follows:

$$
\boldsymbol{\beta}=\left[\begin{array}{l}
\beta_{0} \\
\beta_{1} \\
\beta_{2} \\
\beta_{3}
\end{array}\right]
$$

$$
\boldsymbol{y}=\left[\begin{array}{c}
y^{1} \\
\cdots \\
y^{i} \\
\cdots \\
y^{M}
\end{array}\right]
$$

So the model could be written as:

$$
y^{\prime}=F \beta
$$

The cost function can be written as: ( $M$ is the total number of chromosomes in one patient):

$$
J=\frac{1}{M}\left(\boldsymbol{y}-\boldsymbol{y}^{\prime}\right)^{T}\left(\boldsymbol{y}-\boldsymbol{y}^{\prime}\right)
$$


If we minimize this function, we can get the solution for $\boldsymbol{\beta}$ as:

$$
\boldsymbol{\beta}=\left(\boldsymbol{F}^{T} \boldsymbol{F}\right)^{-1}\left(\boldsymbol{F}^{T} \boldsymbol{y}\right)
$$




\section{CHAPTER 6}

\section{MATERIALS AND METHODS}

We searched for reports that included whole-genome sequencing strategies in their methodology. In order to select suitable studies, we defined a number of requirements, namely studies which were longitudinal, had reported findings on more than one patient, had data which was completely anonymous and publicly available and downloadable in a suitable format. The studies we reviewed had enrolled several patients. The reported results were publicly available and included multiple data on patient's genome during diagnosis from both healthy and cancer tissues, as well as whole-genome sequencing data from affected tissues during disease relapse. Although genome-wide sequencing strategies have been available for more than a decade, there are still a relatively small number of published studies where patients' genome was sequenced after relapse. Most of the published scientific literature on whole-genome sequencing encompasses data comparing the genome changes at nucleotide level from both healthy and affected tissues at one point in time most likely when diagnostic procedures are carried out. As the technologies become less expensive and more widely available, various research groups have started to carry out longitudinal studies where patients' genome is sequenced several times during the disease's progression. 


\subsection{Framework}

We built a framework which is capable of extracting statistical information from the patient's genomic data. Our framework works with the whole patient's genomic data from one patient and not with just one chromosome. Thus, given the genomic data from a patient, we proceed with an analysis at the chromosome level of the whole genome. This analysis involves producing statistical data from the mutations present in each chromosome. The input for this first stage is the longitudinal sequencing data from a patient's tumor where mutations have been identified and characterized.

All data to be used needs to be normalized into a standard format as will be presented in the next sections. As more data is obtained from the patients, more training will be provided for our model. Figure 6.1 below shows this in a graphical manner.

\subsection{Patient data}

Whole genome sequencing data from eight patients with acute myeloid leukemia (AML) served as a basis to test our framework. The data was available as supplementary material to an article published in 2012 by Li Ding et al. in the journal Nature [13]. These patients were from five different French-American-British hematologic subtypes, with elapsed times of 235 to 961 days between samples (see Table 6.1 for each individual's information). The goal of the study was to investigate the mutational profiles of primary tumors and determine whether the clonal evolution of mutations contributes to relapse. We provided our framework with longitudinal data for the mutational profiles observed in the genomes of patients in order to generate a prediction of nucleotide changes (mutations), followed by a comparison of those predictions with the actual mutations 
detected by whole-genome sequencing of the tumors after relapse, thus allowing us to measure accuracy and significance of our approach.

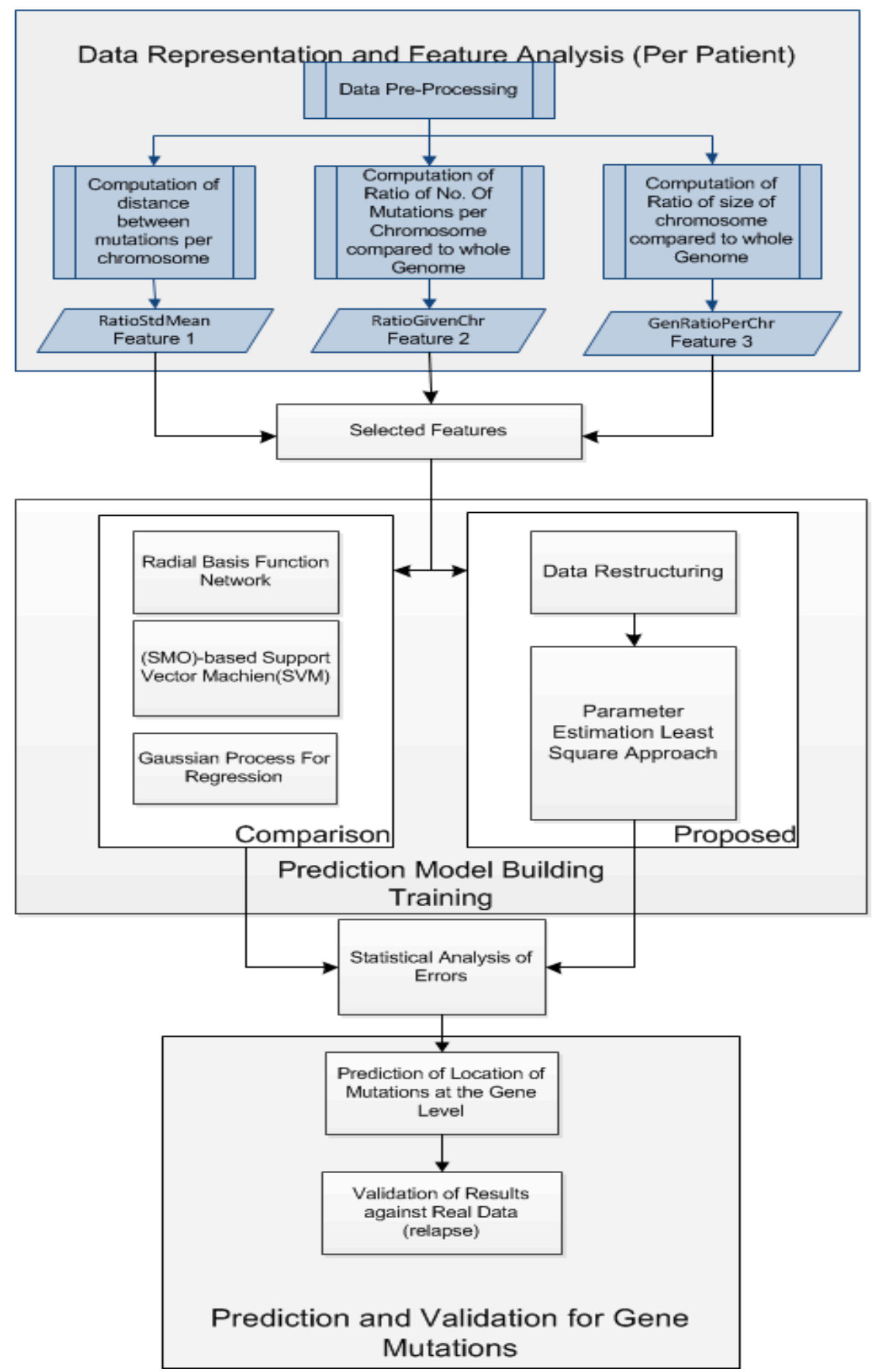

Figure 6.1. Dependency flow graph for our prediction framework. 
Due to different sources of data, and in order to work in a consistent manner, we decided to format all data in a specific manner as explained below. For sequencing data comparisons and evaluations, we aligned the coordinates of input data as well as the coordinates of the resulting predictions to NCBI build 36.3 of human genome assembly. Information corresponding to each patient was labeled with the letters ' $A$ ' through ' $\mathrm{H}$ '. Data was downloaded as plain text files. Files, called "shared.txt", contain the locations of mutations identified. Files, called "relapse.txt", contain the locations of mutations reported after relapse.

\begin{tabular}{|c|c|}
\hline UPN 933124 & Caucasian female late 50s \\
\hline UPN 400220 & Caucasian female, 34 year old \\
\hline UPN 426980 & Caucasian male, 69 year old \\
\hline UPN 452198 & Caucasian male, 55 year old \\
\hline UPN 573988 & Caucasian female, 67 year old \\
\hline UPN 869586 & Caucasian male, 23 year old \\
\hline UPN 758168 & Caucasian female, 25 year old \\
\hline UPN 804168 & Caucasian male, 53 year old \\
\hline
\end{tabular}

Table 6.1. Patient designation, gender and age. 
In Figure 6.2, we show a partial view of the files previously mentioned in order to get an understanding of the way we have been working with them.

In order to map the coordinates of mutations to genes or genetic regions, we downloaded a file from NCBI build 36.3 of the human genome assembly that contains gene names and location ranges per chromosome in a text format and saved it as geneMap.txt. The file is used to determine which genes will be involved depending on the locations of the predicted mutations.

\section{Limitations of Data}

Obtaining patients data is extremely difficult especially because of HIPAA limitations. In our case, we even required data in a longitudinal manner which increased made it even harder. We attended several seminars given by the University of Miami at Jackson's Hospital. During these seminars, medical doctors and other scientists presented their work on cancer research.

Throughout these events we kept asking them for possible sources of these data but their answer was always that the data in a longitudinal manner was very hard to get. Nevertheless, on May 17th, 2013, Dr. James Downing [117], gave a talk titled "The pediatric cancer genome project". Dr. Downing is the Scientific Director for Saint Jude Children's Research Hospital and his talk covered cancer research that was being done by analyzing tumor tissue genomes. After his talk, I asked Dr. Downing for possible sources of longitudinal data based on these genomes, and he suggested me to look for patients with leukemia since it is in this type of disease where most studies had been done in this manner. 
He indicated it would still be hard to access the data but that it was the best route to take.

Following his advice, we continued our research and found the article previously mentioned were the data was finally available.

Simulating data was never an option, since in the medical domain this practice is strictly forbidden since the conclusions derived from here could be misleading and as a result lead to serious medical conclusions.

When we discussed later our work with a domain expert from the UT Southwestern Medical Center, he indicated that our data was very significant since even high quality journals published in top journals use les or comparable amount of data. More on this can be observed in the conclusion of this dissertation.

Our studies are based in the molecular level, as a result, the demographics of the data (e.g. separation by genders) does not apply. Furthermore, if we were to split the demographic aspects such as genders or ages, it would make our data samples even smaller since we would require grouping. Finally these demographic aspects are nominal features and cannot be fit into a regression model directly.

Figure 6.2 shows the format to be used for the geneMap.txt file previously mentioned. 


\begin{tabular}{|ll|}
\hline Chr & Location \\
1 & 108346944 \\
1 & 147392656 \\
1 & 230352346 \\
2 & 25310746 \\
2 & 77165972 \\
2 & 140418446 \\
3 & 182087434 \\
3 & 191103363 \\
3 & 192532267 \\
3 & 196997137 \\
4 & 7394775 \\
4 & 72616906 \\
4 & 119477364 \\
$\ldots$. & \\
22 & 26004684 \\
$X$ & 25476033 \\
$X$ & 105826475 \\
\hline
\end{tabular}

\begin{tabular}{|ll|}
\hline Chr & Location \\
1 & 107357719 \\
1 & 179307551 \\
1 & 218018901 \\
1 & 224145610 \\
2 & 184186967 \\
2 & 213886052 \\
3 & 21310708 \\
3 & 43135962 \\
3 & 135449546 \\
$\ldots .$. & \\
22 & 26004684 \\
$X$ & 25476033 \\
$X$ & 105826475 \\
Arelapse.txt \\
\hline
\end{tabular}

Ashared.txt

Figure 6.2. Sample data files for patient UPN 933124.

\begin{tabular}{|llll|}
\hline chromosome & chr_start & chr_stop & feature_name \\
1 & 815 & 19919 & LOC653635 \\
1 & 42215 & 43358 & OR4G4P \\
1 & 52778 & 53847 & OR4G11P \\
1 & 58954 & 59871 & OR4F5 \\
1 & 77385 & 80096 & LOC100132632 \\
$\cdots$ & & & \\
\hline
\end{tabular}

Figure 6.3. Overview of geneMap.txt.

\subsection{Variables to consider}

Having identified mutations and their locations, our framework will analyze how spaced these mutations are from each other within each chromosome. After obtaining a vector of distances between one mutation and the next, our framework computes statistical analysis 
such as the mean and standard deviation of these distances to identify how these mutations behave. For example, if the standard deviation is very small compared to the mean, this signifies that mutations occur with a similar distance from one another. If that is not the case, then we can see that some mutations are located very much far apart from each other while others are much closer. Thus, the ratio between the mean and the standard deviation (RatioStdMean) of the distances is a critical variable to consider by our framework. A second statistical variable computed by the framework is also the ratio of how many mutations occur within one chromosome when compared to the total number of mutations in the whole genome for a given patient (RatioGivenChr). A third variable, we called GenRatioPerChr, is a standard variable that has also been computed for all experiments. This variable corresponds to the ratio of the chromosome size in nucleotides to the length of the whole human genome assembly also in nucleotides. This is a standard value as it has been obtained from the reference human genome assembly data and it is not specific to a particular patient. Due to the fact that chromosomes are of different sizes, there is a possibility of finding more mutation in larger chromosomes than in shorter ones.

\subsection{Prediction model}

The next step in the framework is about prediction. Our prediction works at two levels. First, we will predict the number of mutations to occur within one chromosome, and then the locations where these mutations will happen. For this contribution, our framework will make usage of the statistical data obtained at the completion of previous stage to 
build a prediction model to determine the number of mutations likely to occur. This prediction model is obtained by utilizing Weka[118] as explained in chapter 4 .

Weka uses an ARFF file (Attribute Relationship File Format) as an input, and then by processing this file, creates the models according to the content of the data and variables involved. The weka ARFF file will have the format indicated in Figure 6.4.

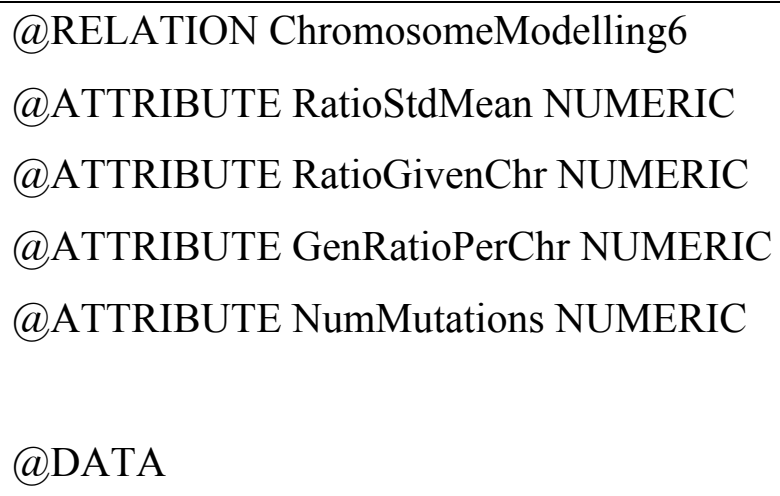

For our purposes, the framework creates an ARFF file per chromosome containing the data for it along with the associated computed variables previously mentioned.

Since the prediction model works at the chromosome level, there will be 24 files corresponding to 22 somatic chromosomes (1 through 22$)$, and one " $\mathrm{X}$ " as well as one "Y" chromosome.

We decided to build a model per chromosome instead of just one model for the whole genome. We carried out a preliminary analysis in which we tried to build a model for the whole genome, but the results were inconclusive. In addition, existing public data is 
available in a per chromosome basis. Furthermore, a generic model did not fit well for all scenarios due to both the intrinsic varying nature and behavior of each chromosome.

For a given chromosome ' $\mathrm{i}$ ' (1-22, and $\mathrm{X}$ as well as $\mathrm{Y})$, our model is of the form:

$$
y^{i}=\beta 1 * F_{1}^{i}+\beta 2 * F_{2}^{i}+\beta 3 * F_{3}^{i}+\beta 0
$$

$y^{i}$ represents the number of mutations we want to predict and $F_{1}^{i}, F_{2}^{i}$, and $F_{3}^{i}$ represent the RatioStdMean, RatioGivenChr and GenRatioPerChr respectively. Finally, $\beta 1, \beta 2$ and $\beta 3$ are the coefficients of the model and $\beta 0$ is the error. Their values are provided by Weka.

These models are obtained by Weka as a String expression and then we parse it and execute each model by inserting the variables of each chromosome for each patient to predict the number of mutations to occur at relapse time.

After generating a model per chromosome, we fit the information obtained from Weka back into the framework. The framework takes this model to execute the prediction of the number of mutations to occur for each chromosome. Once all predicted number of mutations have been computed for each chromosome, the framework takes each individual chromosome and executes the predictions of the locations of the mutations. At this point, since we know the predicted number ' $n$ ' of mutations likely to occur at each chromosome, we perform the algorithm shown in Figure 6.5. 


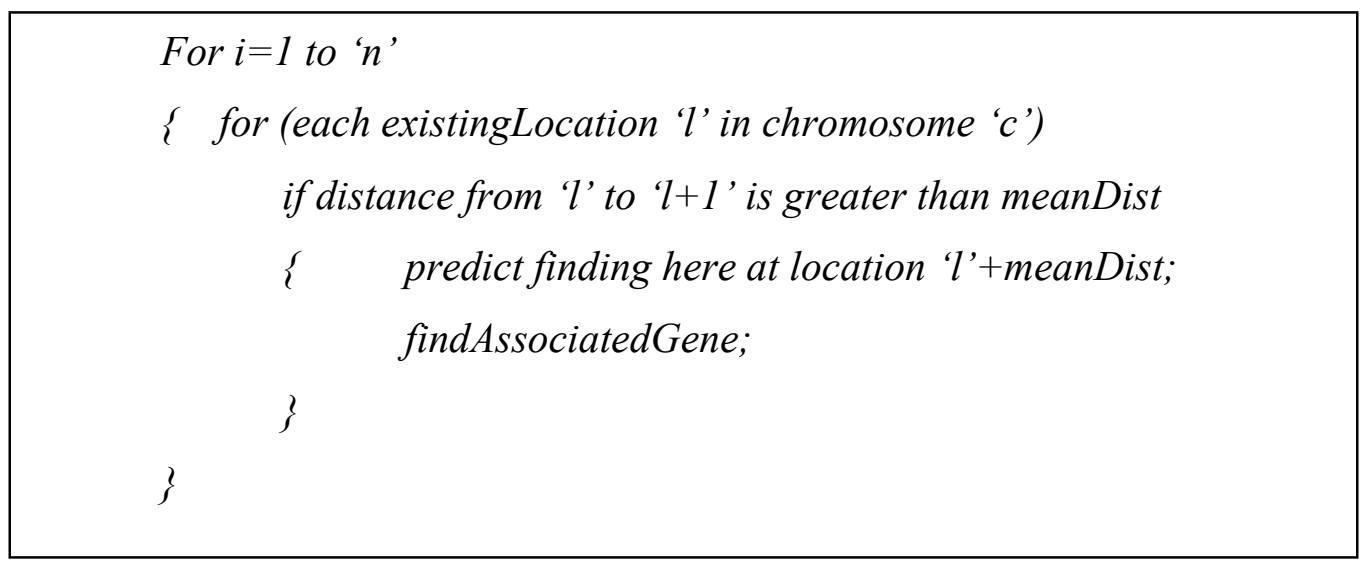

Figure 6.5 Algorithm for finding mutations locations.

We deal with data from mutations in each chromosome at different points in time. In our algorithm, for instance, we utilize existent longitudinal data on mutations during diagnosis and/or treatment to generate a prediction of mutations at a later relapse time. Thus, each location 'l' represents the location of an existent mutation before relapse time. Next, our algorithm checks whether the distance between mutations is greater than the average distance (meanDist), and if that is the case, a mutation will happen within this distance at approximate position of the location ' $\mathrm{l}$ ' + meanDist. Having identified a predicted location for a mutation, we proceed to determine whether it belongs to either an annotated gene or an intergenic region.

Due to the fact that we have the actual number of mutations for all chromosomes, as well as their individual locations identified at relapse time, we are able to compare our predicted results with the experimental data. The results of these comparisons serve as our main criteria to estimate the accuracy of the predictions made by the framework. 


\section{CHAPTER 7}

\section{RESULTS}

\subsection{Prediction of number of mutations per chromosome.}

In order to present, as well as to discuss the results, we selected the data from one of the patients as representative of all (UPN 933124, see Table 7.1 for information on the patient). Data on other patients and their respective predictions are available on the Appendices section. Table 7.1 presents the results for the predictions made by our framework with regards to the number of mutations likely to occur for this given patient. The first column indicates the chromosome analyzed, and the second column indicates the number of mutations observed at the time of relapse (actual number of mutations observed). The third column indicates the predicted number of mutations.

As we can distinguish from Table 7.1, the predicted number of mutations is very close to the observed number of mutations that occurred at the time of relapse in all chromosomes. In order to calculate the accuracy of the prediction, we consider the number of observed mutations versus the number of predicted mutations. We obtained a percent value of the ratio of predicted number of mutations to the total number of observed mutations: $83 \%(35 / 42)$. 


\begin{tabular}{|l|l|l|}
\hline Chromosome & $\begin{array}{l}\text { Observed } \\
\text { Mutations }\end{array}$ & $\begin{array}{l}\text { Predicted } \\
\text { Mutations }\end{array}$ \\
\hline 1 & 4 & 3 \\
\hline 2 & 2 & 2 \\
\hline 3 & 3 & 2 \\
\hline 4 & 1 & 1 \\
\hline 5 & 2 & 2 \\
\hline 6 & 2 & 2 \\
\hline 7 & 3 & 2 \\
\hline 8 & 5 & 4 \\
\hline 9 & 2 & 2 \\
\hline 11 & 3 & 2 \\
\hline 12 & 3 & 2 \\
\hline 13 & 2 & 2 \\
\hline 14 & 2 & 2 \\
\hline 16 & 3 & 2 \\
\hline 17 & 1 & 1 \\
\hline 20 & 2 & 2 \\
\hline 21 & 1 & 1 \\
\hline$X$ & 1 & 1 \\
\hline Total & 42 & 35 \\
\hline Accuracy & $83 \%$ & \\
\cline { 2 - 3 } & &
\end{tabular}

Table 7.1. Number of mutations per chromosome in patient UPN 933124.

\subsubsection{Significance of our experimental data}

The following figures show the error distribution of different patients for the predicted number of mutations at the chromosome level. The $\mathrm{x}$-axis is the predicted value minus ground truth. The y-axis is the counts. It can be seen that the proposed approach gives underestimation of number of mutations on each chromosome of each patient.

In addition, it also can be observed that our miss-predictions focus on negative one, which indicates that the proposed framework is very promising. 


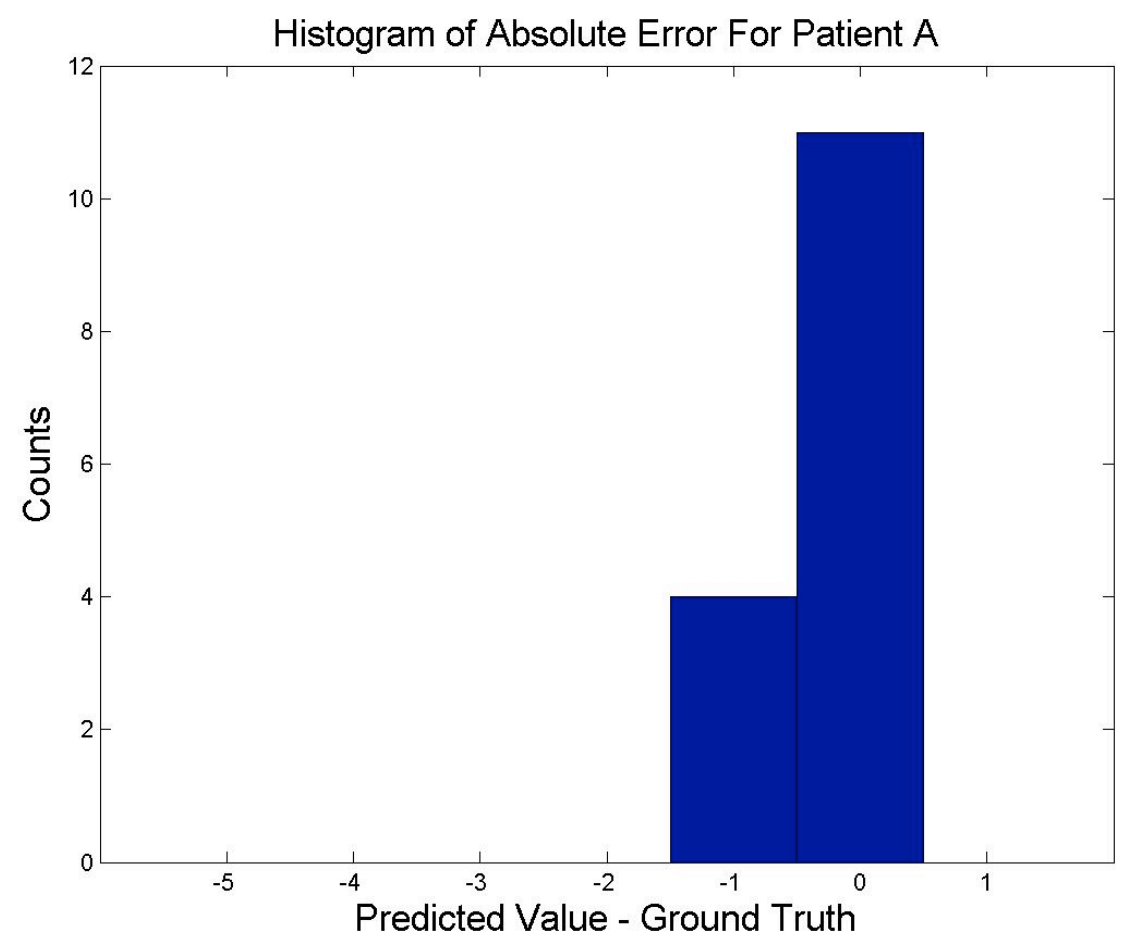

Figure 7.1 Histogram of absolute error for patient A.

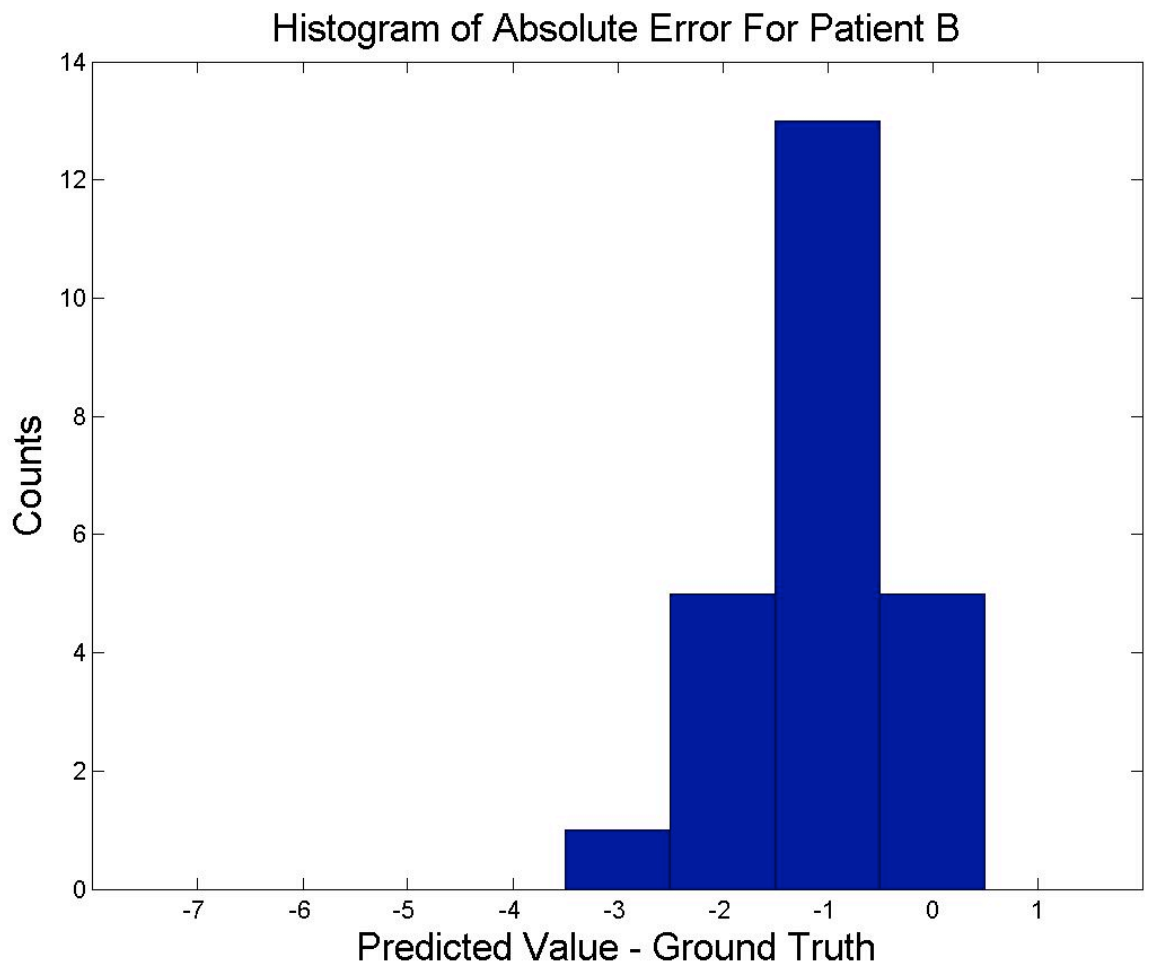

Figure 7.2 Histogram of absolute error for patient B. 


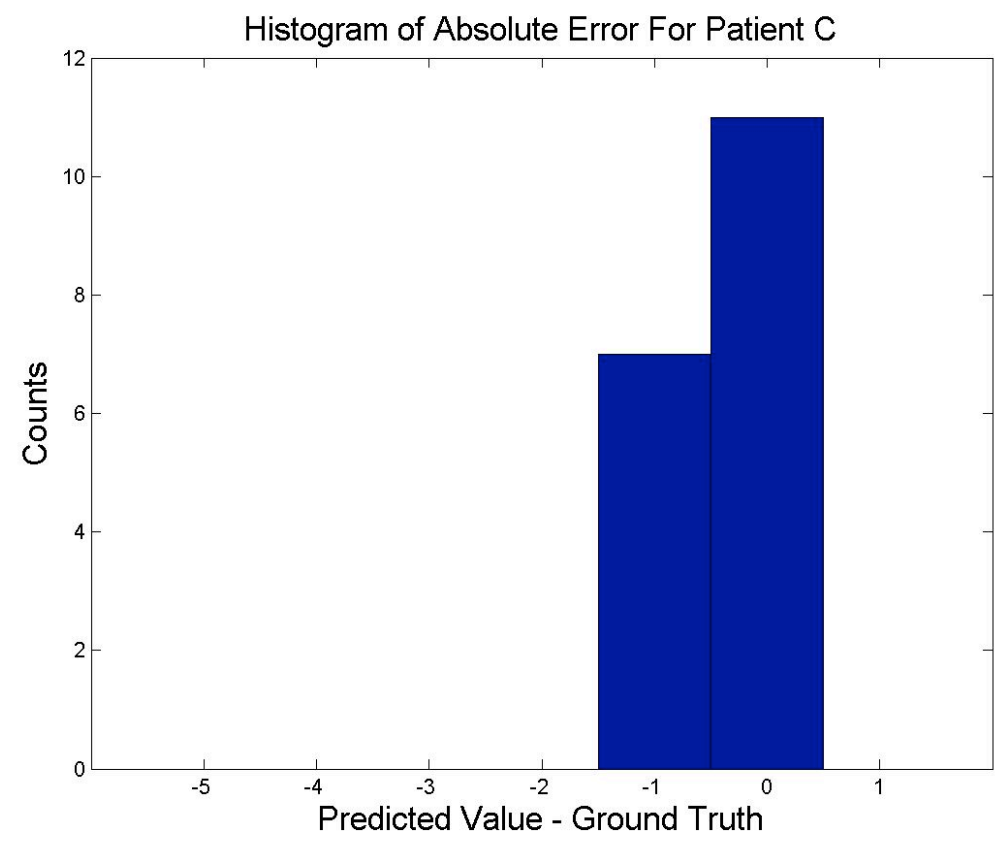

Figure 7.3 Histogram of absolute error for patient C.

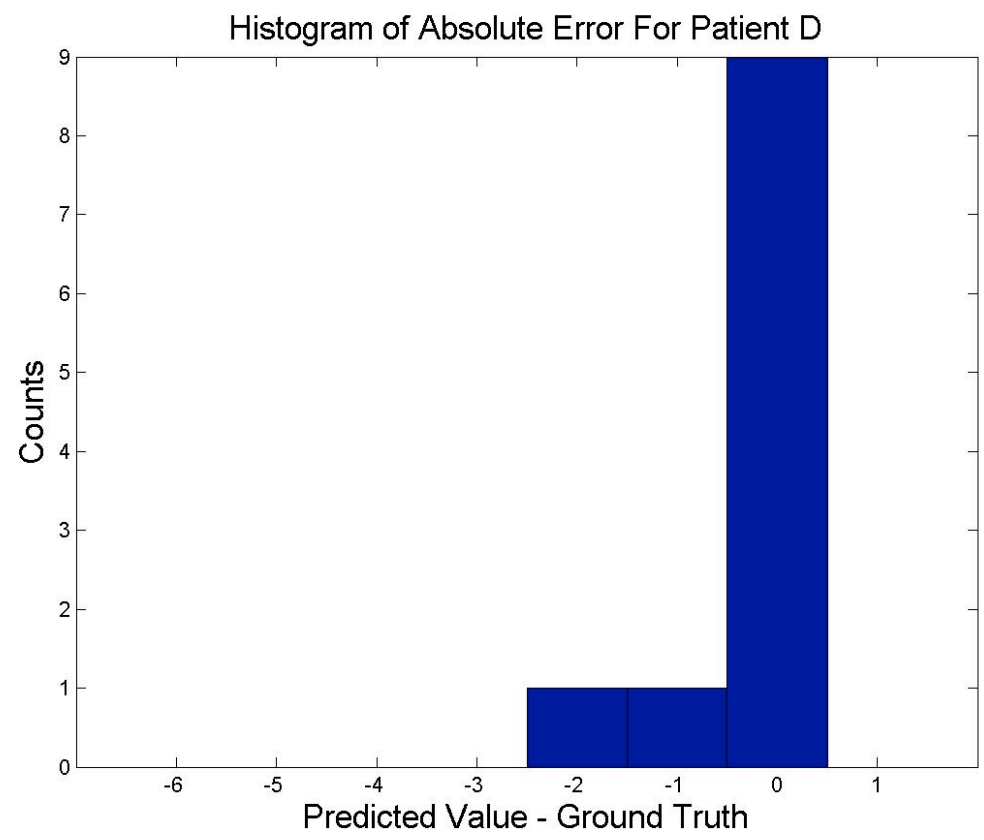

Figure 7.4 Histogram of absolute error for patient D. 


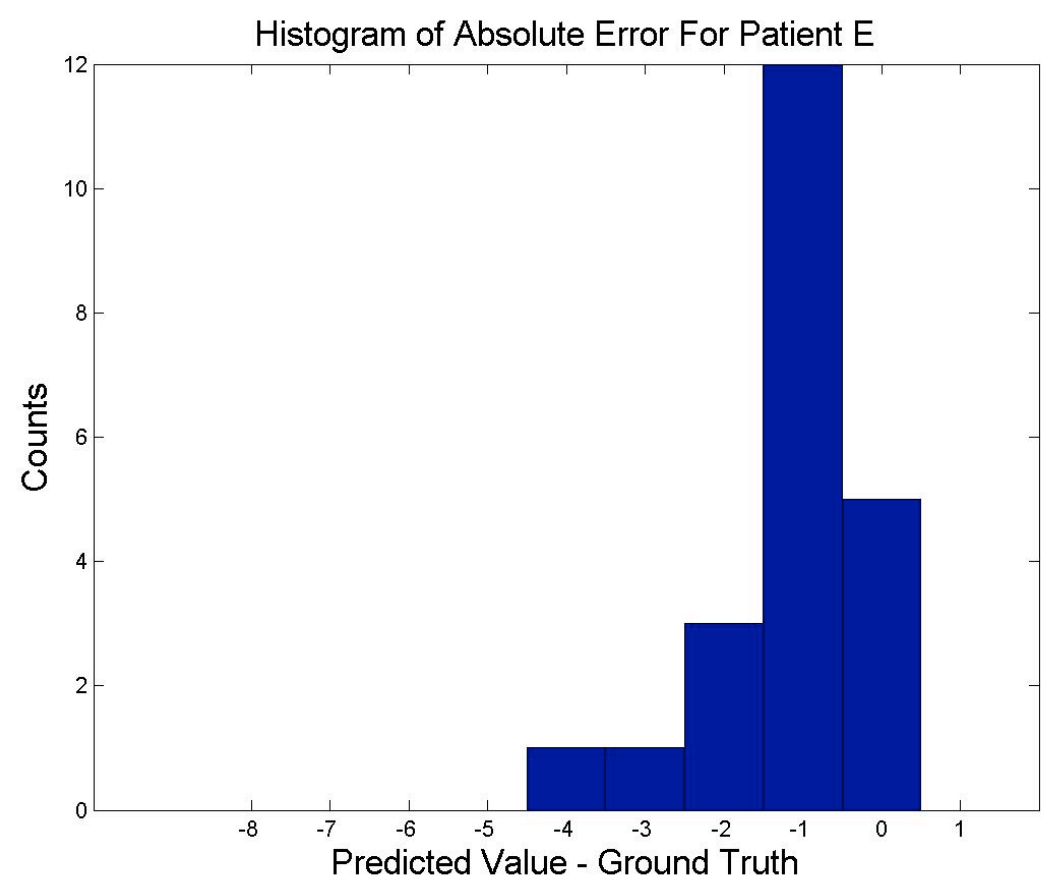

Figure 7.5 Histogram of absolute error for patient E.

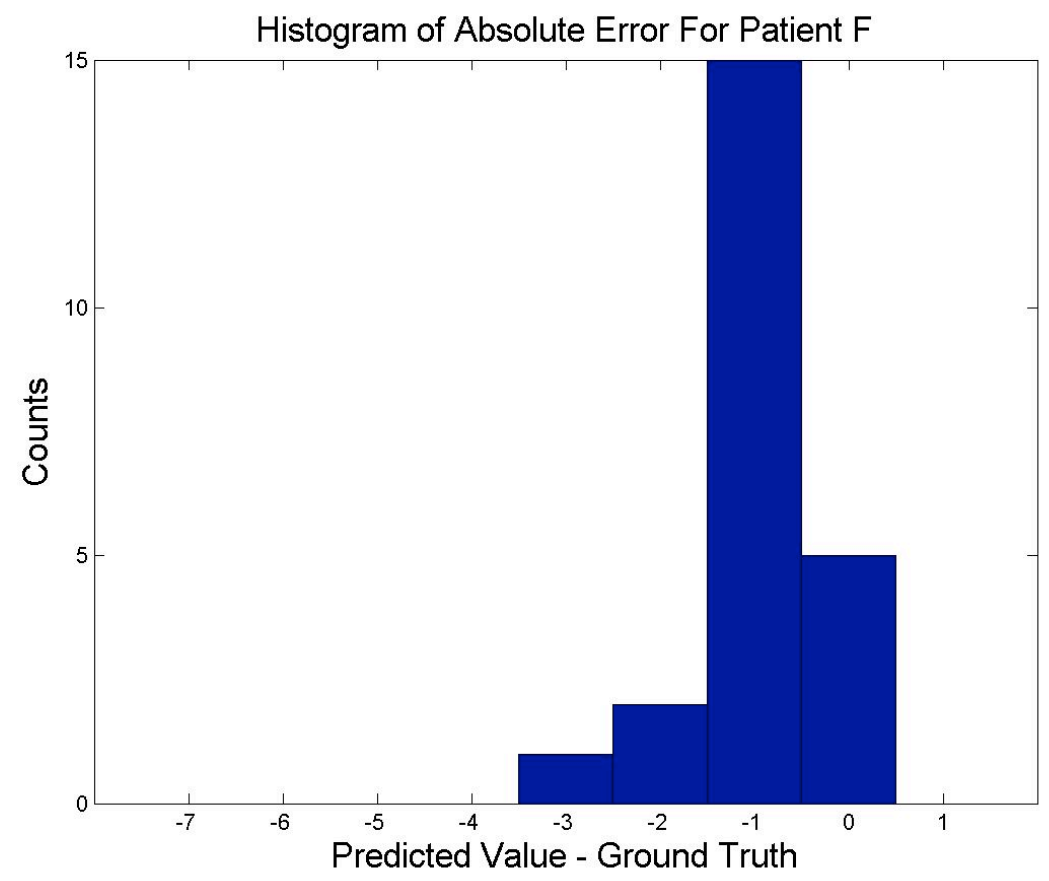

Figure 7.6 Histogram of absolute error for patient $F$. 


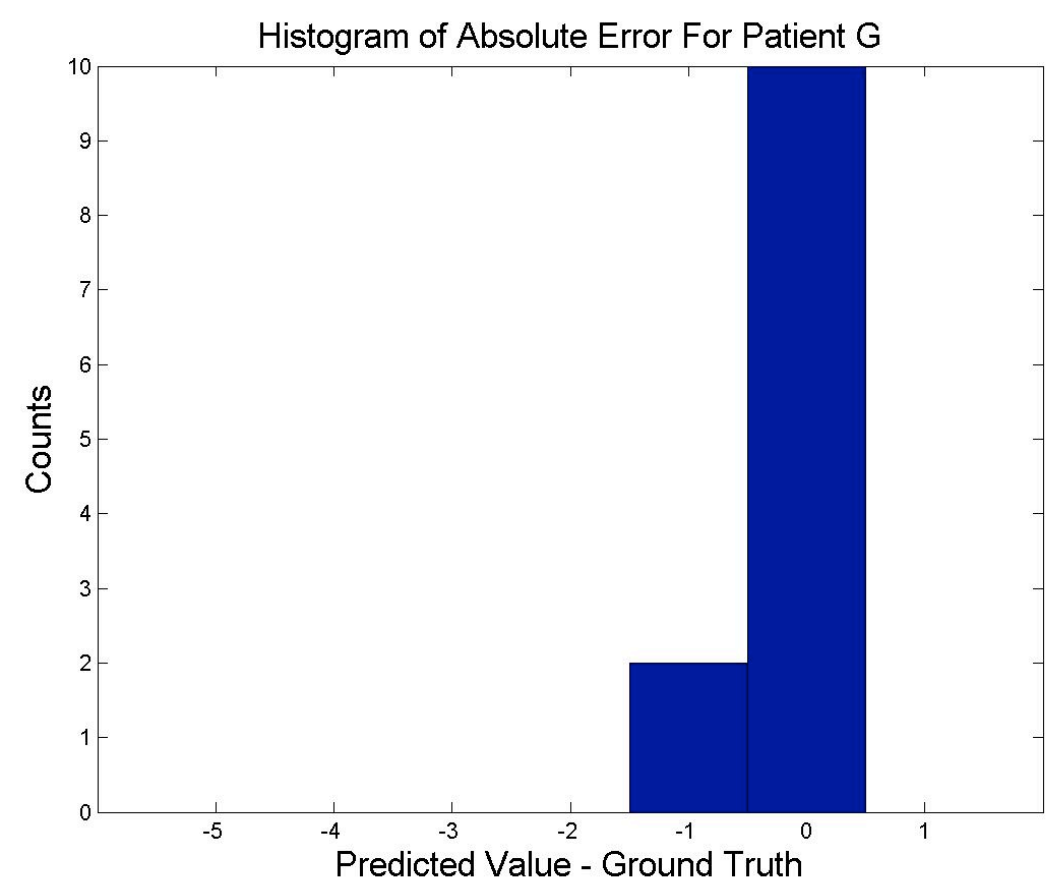

Figure 7.7 Histogram of absolute error for patient G.

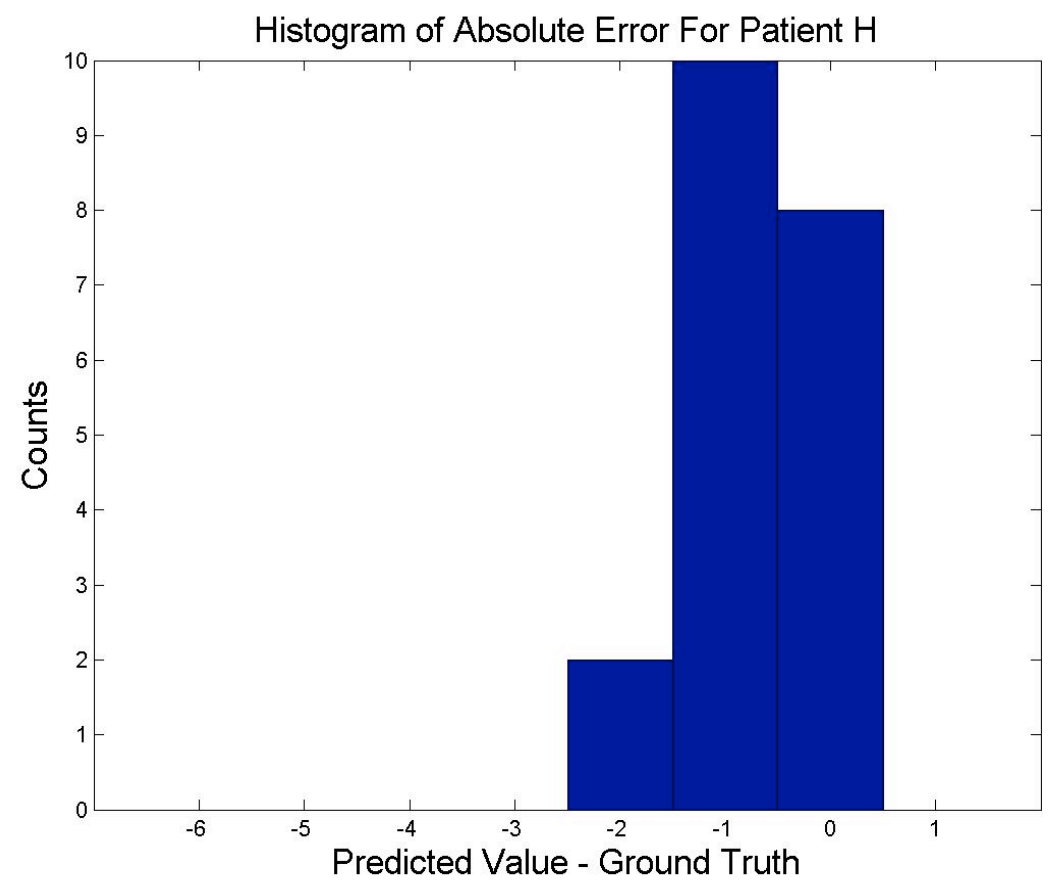

Figure 7.8 Histogram of absolute error for patient $\mathrm{H}$. 
The following figures show the histogram of relative errors. The relative error is defined as the error divided by ground truth number.

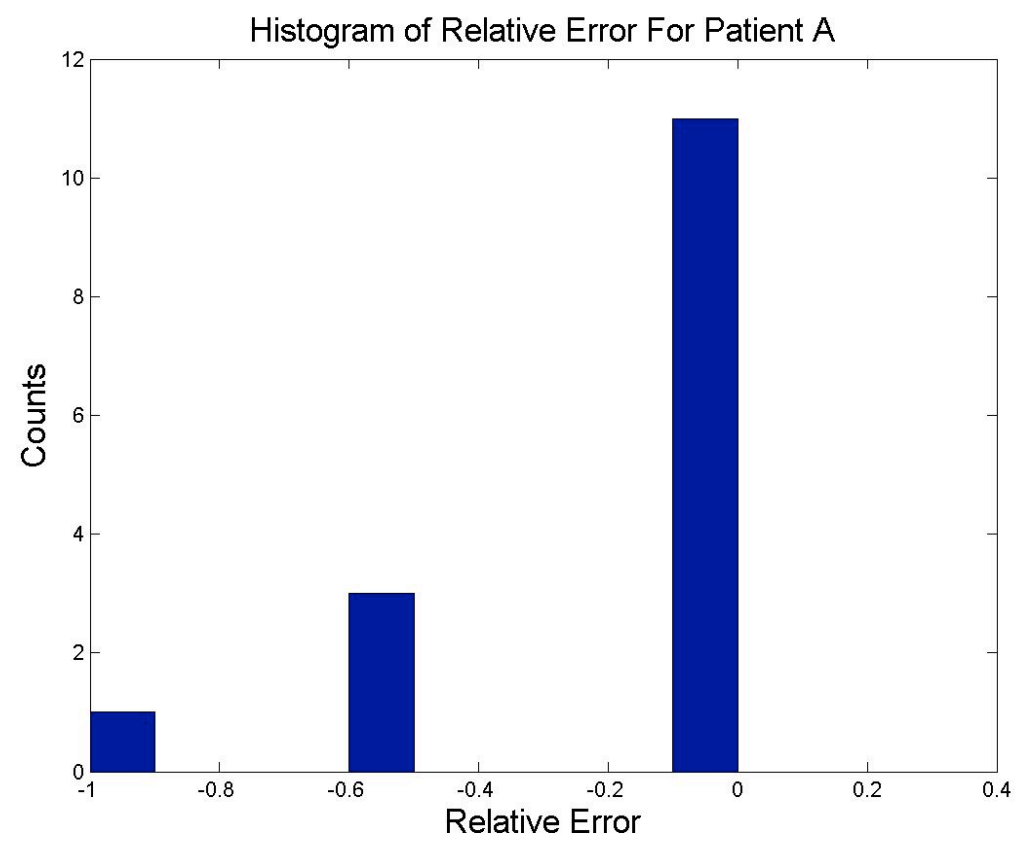

Figure 7.9 Histogram of relative error for patient A.

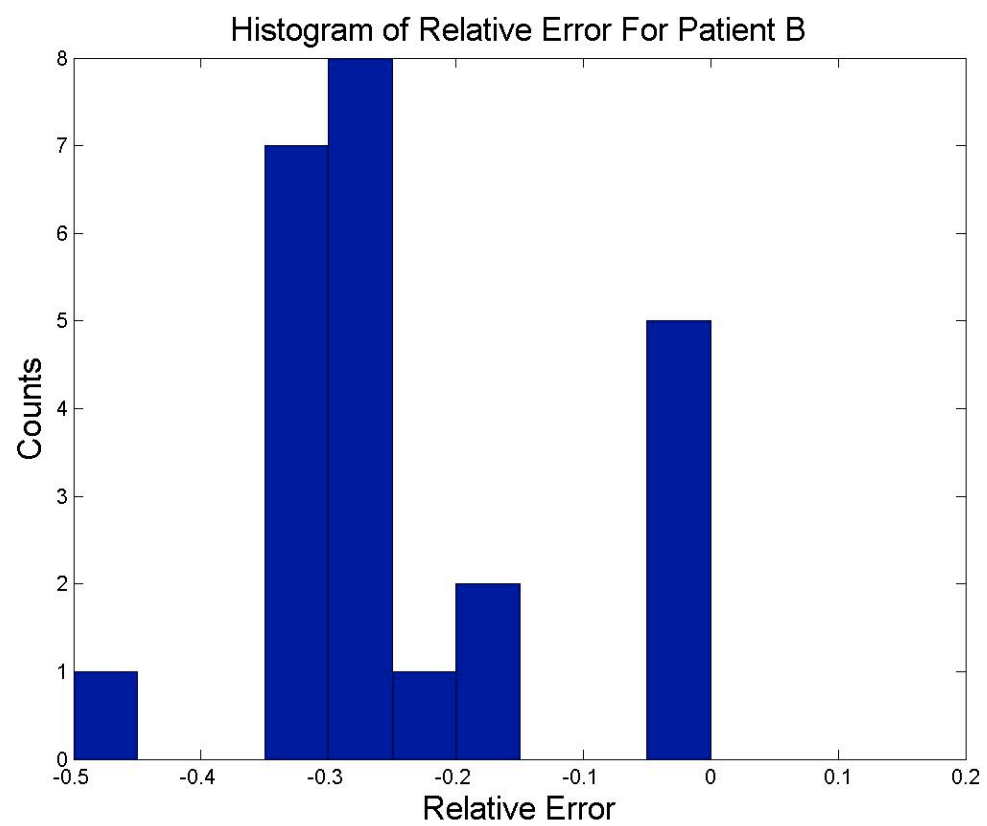

Figure 7.10 Histogram of relative error for patient B. 


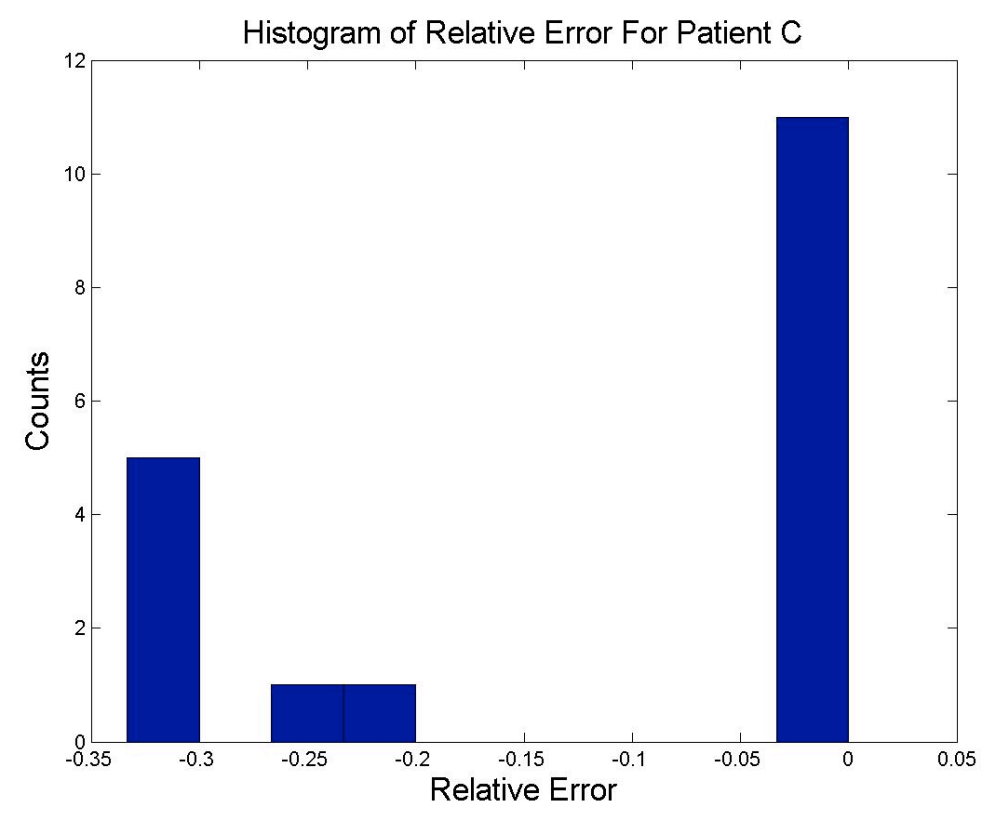

Figure 7.11 Histogram of relative error for patient C.

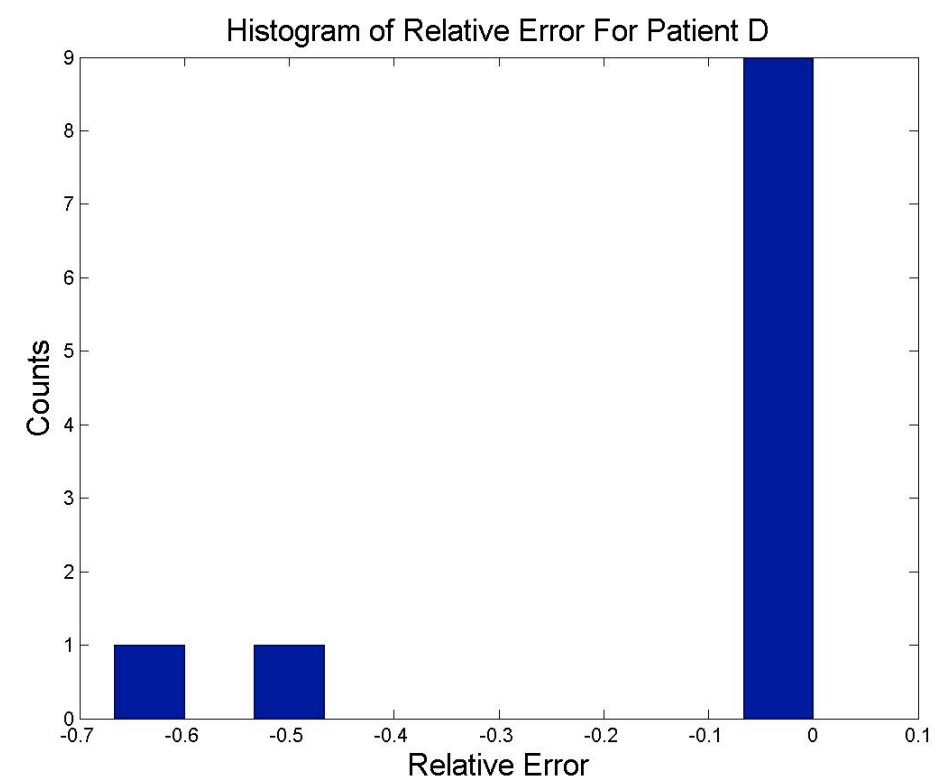

Figure 7.12 Histogram of relative error for patient D. 


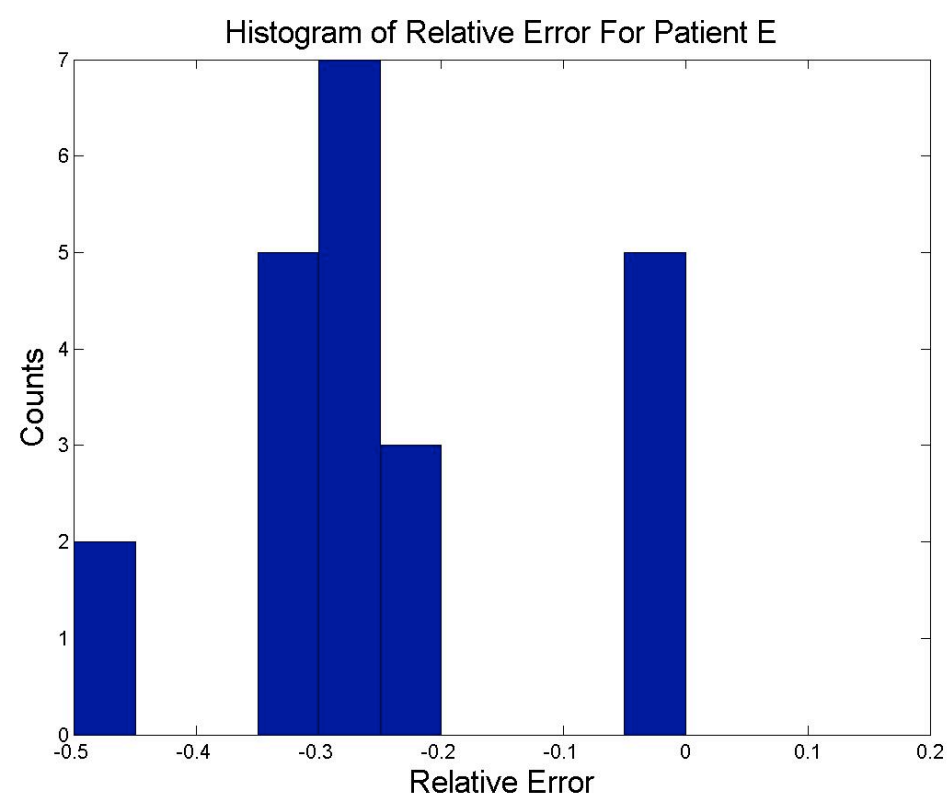

Figure 7.13 Histogram of relative error for patient E.

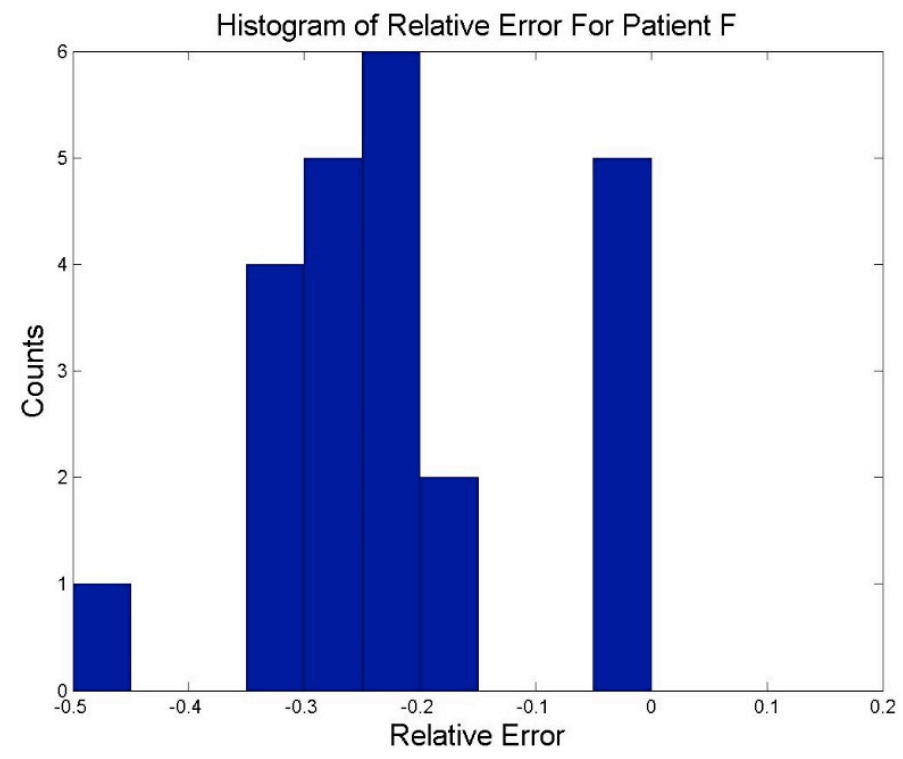

Figure 7.14 Histogram of relative error for patient F. 


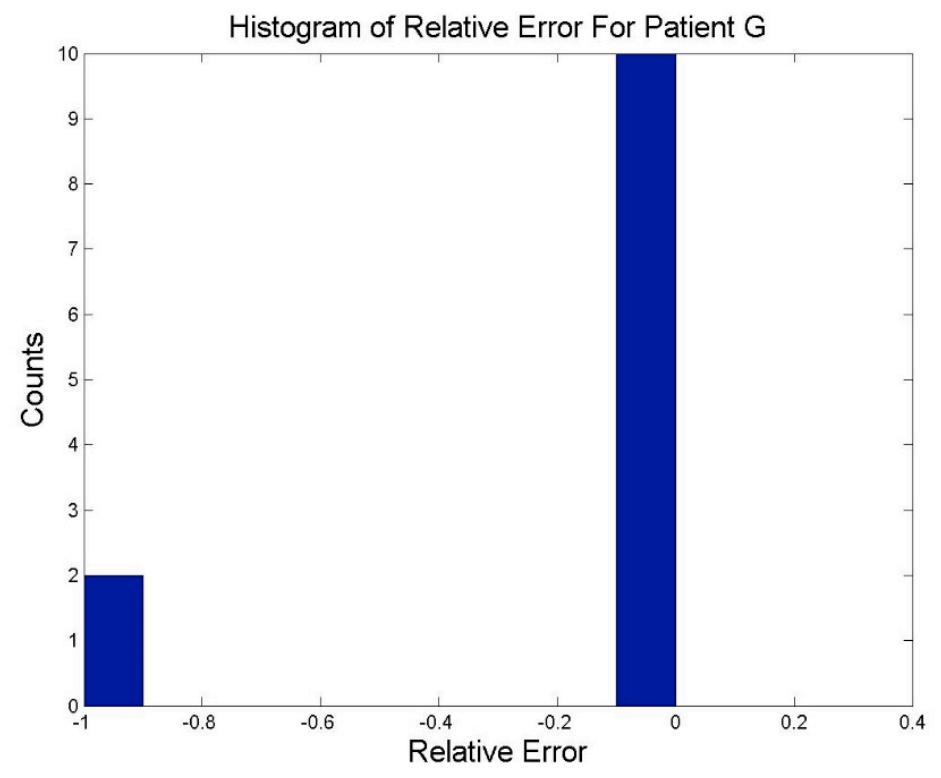

Figure 7.15 Histogram of relative error for patient G.

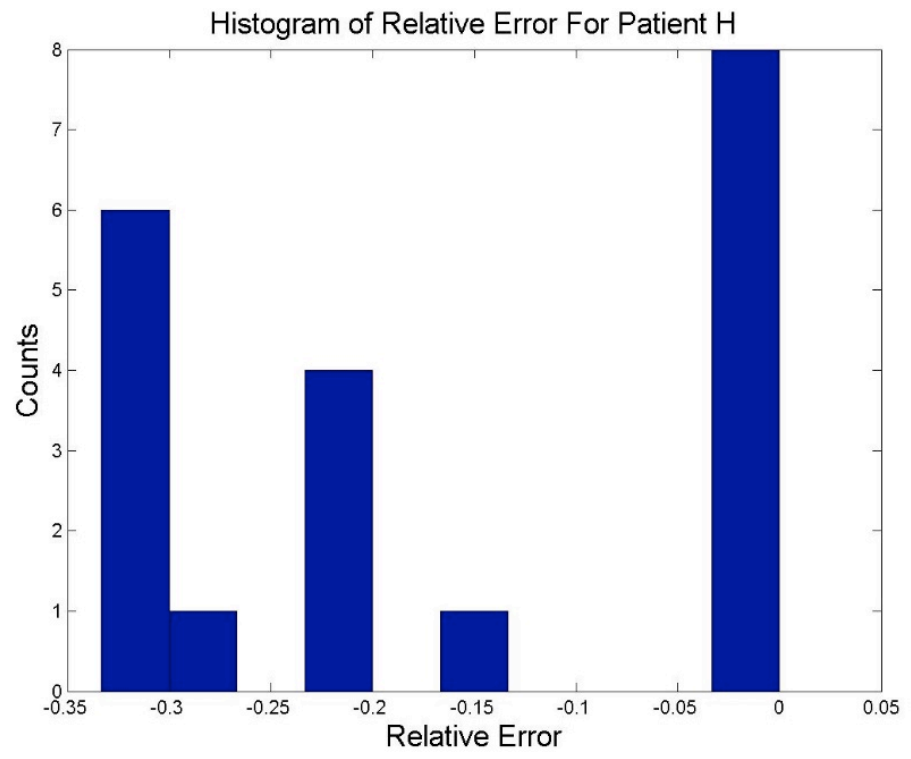

Figure 7.16 Histogram of relative error for patient $\mathrm{H}$. 
After a thorough review of our experimental data on the error distribution, we have the following results:

Observation (1): The x-axis is predicted value minus ground truth. The y-axis is the counts. It can be seen that the proposed approach gives underestimation of number of mutations on each chromosome of each patient.

Observation (2): It also can be observed that our mis-predictions focuses on negative one, which indicates that the proposed computational framework is very promising.

\subsubsection{Analysis of performance measures to evaluate the success of our prediction}

The state of the art evaluation criteria for classification models include accuracy, sensitivity, specificity, precision, true positive value, and true negative values. These metrics are suitable for binary classification problems; however, in our case our research problem is a regression problem and, as a result, the previously mentioned criteria cannot be applied. Consequently, our analysis for accuracy was different as can be observed in the paragraphs below. Detailed statistical analysis of the errors is also provided in Section 7.1.3.

We obtained performance measures to evaluate the success of our prediction. For this, we

defined $p_{1}, p_{2}, p_{3}, \ldots, p_{n}$ as the numeric value of the prediction at the $i^{\text {th }}$ instance and we define $\mathrm{a}_{1}, \mathrm{a}_{2}, \mathrm{a}_{3}, \ldots$, an as the actual values at the $\mathrm{i}^{\text {th }}$ instance.

The correlation coefficient measures the statistical correlation between a's and p's. The closer we get to 1 for this value, the better correlated results. 
Correlation Coefficient $=\mathrm{S}_{\mathrm{PA}} / \sqrt{S_{p} S_{A}}$ where $\mathrm{S}_{\mathrm{PA}}=\frac{\sum_{i}\left(p_{i}-\bar{p}\right)\left(a_{i}-\bar{a}\right)}{n-1}$

$$
\mathrm{S}_{\mathrm{P}}=\frac{\sum_{i}\left(p_{i}-\bar{p}\right)^{2}}{n-1} \text { and } \mathrm{S}_{\mathrm{A}}=\frac{\sum_{i}\left(a_{i}-\bar{a}\right)^{2}}{n-1}
$$

We obtained the mean absolute error which represents the average of the individual errors without considering their signs.

Mean Absolute Error $=\frac{\left|p_{1}-a_{1}\right|+\cdots+\left|p_{n}-a_{n}\right|}{n}$

The root mean squared error (most widely used measure). It represents the square root of the mean squared error and presents the same dimensions as the predicted value itself.

Root mean squared error $=\sqrt{\frac{\left(p_{1}-a_{1}\right)^{2}+\cdots+\left(p_{n}-a_{n}\right)^{2}}{n}}$

"Simple predictor" is the average of all actual values given by the training data.

The relative absolute error computes the total absolute error with the same kind of normalization. Relative errors are normalized by the error of the simple predictor that predicts average values.

Relative absolute error $=\frac{\left|p_{1}-a_{1}\right|+\cdots+\left|p_{n}-a_{n}\right|}{\left|a_{1}-\bar{a}\right|+\cdots+\left|a_{n}-\bar{a}\right|}$

Finally, the relative squared error represents the scenario where a simple predictor had been used. It is given by total squared error normalized by dividing it by the total squared error of the default predictor.

Relative squared error $=\frac{\left(p_{1}-a_{1}\right)^{2}+\cdots+\left(p_{n}-a_{n}\right)^{2}}{\left(a_{1}-\bar{a}\right)^{2}+\cdots+\left(a_{n}-\bar{a}\right)^{2}}$

Finally, all these values are shown in table 7.2 for Patient A. The respective tables for other patients can be found at the Appendices. 


\begin{tabular}{|c|r|r|r|r|r|}
\hline Chromosome & $\begin{array}{c}\text { Correlation } \\
\text { coefficient }\end{array}$ & $\begin{array}{c}\text { Mean } \\
\text { absolute } \\
\text { error }\end{array}$ & $\begin{array}{c}\text { Root } \\
\text { mean } \\
\text { squared } \\
\text { error }\end{array}$ & $\begin{array}{c}\text { Relative } \\
\text { absolute } \\
\text { error }\end{array}$ & $\begin{array}{c}\text { Root } \\
\text { relative } \\
\text { squared } \\
\text { error }\end{array}$ \\
\hline $\mathbf{1}$ & 0.9412 & 0.6797 & 0.9251 & $27.19 \%$ & $33.78 \%$ \\
\hline $\mathbf{2}$ & 0.9999 & 0.015 & 0.0181 & $1.20 \%$ & $1.22 \%$ \\
\hline $\mathbf{3}$ & 0.9994 & 0.1137 & 0.1144 & $3.79 \%$ & $3.41 \%$ \\
\hline $\mathbf{4}$ & 0.9769 & 0.4618 & 0.6322 & $18.47 \%$ & $21.37 \%$ \\
\hline $\mathbf{5}$ & 0.9475 & 0.6512 & 0.8757 & $26.05 \%$ & $31.98 \%$ \\
\hline $\mathbf{6}$ & 0.9998 & 0.0702 & 0.0752 & $2.16 \%$ & $2.15 \%$ \\
\hline $\mathbf{7}$ & 0.9826 & 0.2975 & 0.3562 & $18.31 \%$ & $18.55 \%$ \\
\hline $\mathbf{8}$ & 0.9972 & 0.1895 & 0.2338 & $6.89 \%$ & $7.51 \%$ \\
\hline $\mathbf{9}$ & 0.9403 & 0.5651 & 0.7798 & $28.26 \%$ & $34.03 \%$ \\
\hline $\mathbf{1 0}$ & 0.9896 & 0.5765 & 0.5954 & $15.37 \%$ & $14.36 \%$ \\
\hline $\mathbf{1 1}$ & 0.9565 & 0.4473 & 0.5969 & $25.56 \%$ & $29.17 \%$ \\
\hline $\mathbf{1 2}$ & 0.9946 & 0.2674 & 0.3315 & $8.91 \%$ & $10.36 \%$ \\
\hline $\mathbf{1 3}$ & 0.9999 & 0.0314 & 0.0314 & $2.09 \%$ & $1.48 \%$ \\
\hline $\mathbf{1 4}$ & 1 & 0.0014 & 0.0016 & $0.16 \%$ & $0.15 \%$ \\
\hline $\mathbf{1 5}$ & 0.9995 & 0.122 & 0.1225 & $3.49 \%$ & $3.22 \%$ \\
\hline $\mathbf{1 6}$ & 0.9609 & 0.2272 & 0.3094 & $22.72 \%$ & $27.68 \%$ \\
\hline $\mathbf{1 7}$ & 0.9987 & 0.155 & 0.1554 & $5.64 \%$ & $5.13 \%$ \\
\hline $\mathbf{1 8}$ & 0.9914 & 0.3529 & 0.3854 & $12.83 \%$ & $13.08 \%$ \\
\hline $\mathbf{1 9}$ & 0.9885 & 0.3556 & 0.3766 & $15.80 \%$ & $15.14 \%$ \\
\hline $\mathbf{2 0}$ & 0.9702 & 0.4256 & 0.5547 & $21.28 \%$ & $24.21 \%$ \\
\hline $\mathbf{2 1}$ & 0.9959 & 0.3202 & 0.376 & $8.54 \%$ & $9.07 \%$ \\
\hline $\mathbf{2 2}$ & 0.9786 & 0.4876 & 0.5916 & $19.50 \%$ & $20.60 \%$ \\
\hline $\mathbf{X}$ & 0.9922 & 0.2044 & 0.2841 & $10.90 \%$ & $12.47 \%$ \\
\hline $\mathbf{Y}$ & 0.9992 & 0.082 & 0.1047 & $3.64 \%$ & $3.91 \%$ \\
\hline & & & & & \\
\hline & & & & \\
\hline & & & & & \\
\hline & & & &
\end{tabular}

Table 7.2. Table for performance measures for our prediction model.

\subsubsection{Significance, stability and statistical analysis of model}

In this study, we achieved promising results. The correlation coefficient, which indicates the degree of matching of the proposed framework with real data, is $0.9816 \pm 0.009$ with $95 \%$ confidence interval. For mean absolute error, we achieved $0.2958 \pm 0.087$ with $95 \%$ confidence interval.

Here we also provide a statistical analysis for all errors across all chromosomes for 
Patient A. The specific algorithm is given as follows. Because the data collected are limited in our case and the population standard deviation is unknown, we use the Student's $t$-distribution to estimate the confidence interval. For one variable $a$ such as the correlation coefficient, $n$ is the number of observations ( $n=24$ in our case). The algorithm to compute the confidence interval $c f$ is as follows.

\section{ALGORITHM}

Begin

Step 1: Compute the average value of $a$, which is $\bar{a}$;

Step 2: Compute the standard deviation of $a$, which is $s$;

Step 3: Set a confidence interval, here we used $95 \%$, which is a golden standard in statistics community.

Step 4: Compute a $T \_$multiplier, which is the inverse of Students' $t$ cumulative distribution function using the confidence interval and the freedom of $n-1$

Step 5: Compute a $c f$ value

$$
c f=T \_ \text {multiplier } * s / \sqrt{n}
$$

Step 6: The confidence interval is $[\bar{a}-c f, \bar{a}+c f]$

End

The table of the statistics of the first patient is in the below table. As mentioned earlier and as it can be observed in Table 7.3, for patient A, we have correlation coefficient as $0.9834 \pm 0.0084$ with $95 \%$ confidence interval. Additionally, for mean absolute error, we 
achieved $0.2958 \pm 0.087$ with $95 \%$ confidence interval. Tables 7.3 through 7.10 show these measures as well for all other patients. Finally, table 7.11 shows the statistical analysis for all patients.

\begin{tabular}{|c|c|c|c|c|c|}
\hline & mean & std & cf & lower bound & upper bound \\
\hline Correlation coefficient & 0.9834 & 0.0200 & 0.0084 & 0.9749 & 0.9918 \\
\hline Mean absolute error & 0.2958 & 0.2061 & 0.0870 & 0.2088 & 0.3829 \\
\hline Root mean squared error & 0.3678 & 0.2736 & 0.1155 & 0.2523 & 0.4834 \\
\hline Relative absolute error & 0.1286 & 0.0927 & 0.0391 & 0.0895 & 0.1678 \\
\hline Root relative squared error & 0.1433 & 0.1123 & 0.0474 & 0.0959 & 0.1907 \\
\hline
\end{tabular}

Table 7.3. Statistical analysis of errors for patient A.

\begin{tabular}{|c|c|c|c|c|c|}
\hline & mean & std & cf & lower bound & upper bound \\
\hline Correlation coefficient & 0.9807 & 0.0193 & 0.0081 & 0.9726 & 0.9888 \\
\hline Mean absolute error & 0.3000 & 0.2065 & 0.0872 & 0.2128 & 0.3872 \\
\hline Root mean squared error & 0.3730 & 0.2731 & 0.1153 & 0.2577 & 0.4884 \\
\hline Relative absolute error & 0.1336 & 0.0922 & 0.0390 & 0.0947 & 0.1726 \\
\hline Root relative squared error & 0.1484 & 0.1126 & 0.0475 & 0.1009 & 0.1960 \\
\hline
\end{tabular}

Table 7.4. Statistical analysis of errors for patient B. 


\begin{tabular}{|c|c|c|c|c|c|}
\hline & mean & std & cf & lower bound & upper bound \\
\hline Correlation coefficient & 0.9821 & 0.0178 & 0.0075 & 0.9746 & 0.9897 \\
\hline Mean absolute error & 0.3009 & 0.2056 & 0.0868 & 0.2141 & 0.3877 \\
\hline Root mean squared error & 0.3739 & 0.2737 & 0.1156 & 0.2583 & 0.4895 \\
\hline Relative absolute error & 0.1329 & 0.0934 & 0.0394 & 0.0935 & 0.1724 \\
\hline Root relative squared error & 0.1477 & 0.1123 & 0.0474 & 0.1003 & 0.1951 \\
\hline
\end{tabular}

Table 7.5. Statistical analysis of errors for patient C.

\begin{tabular}{|c|c|c|c|c|c|}
\hline & mean & std & cf & lower bound & upper bound \\
\hline Correlation coefficient & 0.9826 & 0.0168 & 0.0071 & 0.9755 & 0.9897 \\
\hline Mean absolute error & 0.3005 & 0.2061 & 0.0870 & 0.2135 & 0.3876 \\
\hline Root mean squared error & 0.3732 & 0.2735 & 0.1155 & 0.2578 & 0.4887 \\
\hline Relative absolute error & 0.1345 & 0.0923 & 0.0390 & 0.0955 & 0.1735 \\
\hline Root relative squared error & 0.1468 & 0.1129 & 0.0477 & 0.0991 & 0.1944 \\
\hline
\end{tabular}

Table 7.6. Statistical analysis of errors for patient D. 


\begin{tabular}{|c|c|c|c|c|c|}
\hline & mean & std & cf & lower bound & upper bound \\
\hline Correlation coefficient & 0.9802 & 0.0195 & 0.0082 & 0.9720 & 0.9885 \\
\hline Mean absolute error & 0.2997 & 0.2050 & 0.0866 & 0.2132 & 0.3863 \\
\hline Root mean squared error & 0.3725 & 0.2749 & 0.1161 & 0.2564 & 0.4886 \\
\hline Relative absolute error & 0.1336 & 0.0924 & 0.0390 & 0.0946 & 0.1726 \\
\hline Root relative squared error & 0.1476 & 0.1121 & 0.0473 & 0.1003 & 0.1949 \\
\hline
\end{tabular}

Table 7.7. Statistical analysis of errors for patient E.

\begin{tabular}{|c|c|c|c|c|c|}
\hline & mean & std & cf & lower bound & upper \\
bound \\
\hline Correlation coefficient & 0.9807 & 0.0197 & 0.0083 & 0.9724 & 0.9891 \\
\hline Mean absolute error & 0.3012 & 0.2065 & 0.0872 & 0.2140 & 0.3884 \\
\hline Root mean squared error & 0.3719 & 0.2740 & 0.1157 & 0.2562 & 0.4876 \\
\hline Root relative squared & 0.1324 & 0.0919 & 0.0388 & 0.0936 & 0.1712 \\
\hline error & 0.1487 & 0.1125 & 0.0475 & 0.1012 & 0.1962 \\
\hline
\end{tabular}

Table 7.8. Statistical analysis of errors for patient F. 


\begin{tabular}{|c|c|c|c|c|c|}
\hline & mean & std & cf & lower bound & upper bound \\
\hline Correlation coefficient & 0.9814 & 0.0191 & 0.0081 & 0.9734 & 0.9895 \\
\hline Mean absolute error & 0.2997 & 0.2062 & 0.0871 & 0.2126 & 0.3868 \\
\hline Root mean squared error & 0.3722 & 0.2732 & 0.1154 & 0.2568 & 0.4876 \\
\hline Relative absolute error & 0.1337 & 0.0930 & 0.0393 & 0.0944 & 0.1730 \\
\hline Root relative squared error & 0.1481 & 0.1120 & 0.0473 & 0.1008 & 0.1954 \\
\hline
\end{tabular}

Table 7.9 Statistical analysis of errors for patient G.

\begin{tabular}{|c|c|c|c|c|c|}
\hline & mean & std & cf & lower bound & upper bound \\
\hline Correlation coefficient & 0.9818 & 0.0190 & 0.0080 & 0.9738 & 0.9899 \\
\hline Mean absolute error & 0.3019 & 0.2067 & 0.0873 & 0.2146 & 0.3892 \\
\hline Root mean squared error & 0.3721 & 0.2733 & 0.1154 & 0.2567 & 0.4875 \\
\hline Relative absolute error & 0.1343 & 0.0922 & 0.0389 & 0.0953 & 0.1732 \\
\hline Root relative squared error & 0.1479 & 0.1131 & 0.0477 & 0.1001 & 0.1956 \\
\hline
\end{tabular}

Table 7.10. Statistical analysis of errors for patient $\mathrm{H}$. 


\begin{tabular}{|c|c|c|c|c|c|}
\hline & mean & std & cf & lower bound & upper bound \\
\hline Correlation coefficient & 0.9816 & 0.0011 & 0.0009 & 0.9808 & 0.9825 \\
\hline Mean absolute error & 0.3000 & 0.0018 & 0.0015 & 0.2984 & 0.3015 \\
\hline Root mean squared error & 0.3721 & 0.0018 & 0.0015 & 0.3705 & 0.3736 \\
\hline Relative absolute error & 0.1330 & 0.0019 & 0.0016 & 0.1314 & 0.1345 \\
\hline Root relative squared error & 0.1473 & 0.0017 & 0.0014 & 0.1459 & 0.1487 \\
\hline
\end{tabular}

Table 7.11. Statistical analysis of all patients.

\subsubsection{Observations}

Table 7.11 gives a summary of all eight patients. As indicated in the table, our method is relatively stable across different patients. For example, for the eight patients, the confidence interval with $95 \%$ for the correlation coefficient is $0.9816 \pm 0.009$. Since the eight patients are chosen randomly, these experimental results indicate that our model fitting could give around 0.9816 of correlation coefficient measurement. As we know that 1 indicates the perfect performance. This measurement indicates that our model fits the data relatively well.

Below, we can also see the coefficients obtained for the models at each chromosome per patient. We tried to find some patterns among across different chromosomes within the same patient and then also tried to find patterns across patients and we could not identify any significant patterns. 
For the purpose of showing how the models look like, we show all tables that contain the coefficients for the chromosome models for each patient.

From the model $y^{i}=\beta 1 * F_{1}^{i}+\beta 2 * F_{2}^{i}+\beta 3 * F_{3}^{i}+\beta 0$,

$\beta 1, \beta 2$, and $\beta 3$ are the coefficients for RatioStdMean, RatioGIvenChr and

ChrToGenomRatio respectively. $\beta 0$ is the error used to adjust the model.

\begin{tabular}{|r|r|r|r|}
\hline chr & \multicolumn{1}{|c|}{$\boldsymbol{\beta 1}$} & $\boldsymbol{\beta}$ & \multicolumn{1}{c|}{$\boldsymbol{\beta} \mathbf{3}$} \\
\hline 1 & -0.0058 & -0.9408 & 0.00000012 \\
\hline 2 & 0.0044 & -1.1347 & -0.00000002 \\
\hline 3 & 0.0045 & 4.3818 & 0.00000015 \\
\hline 4 & 0.0048 & 5.3997 & 0.00000032 \\
\hline 5 & -0.0124 & -6.074 & 0.00000017 \\
\hline 6 & 0.006 & 4.4676 & 0.00000009 \\
\hline 7 & 0.0125 & 3.0178 & 0.00000008 \\
\hline 8 & 0.0084 & 7.6347 & 0.00000013 \\
\hline 9 & 0.0127 & -6.1969 & -0.00000014 \\
\hline 10 & -0.0051 & 6.0138 & -0.00000016 \\
\hline 11 & 0.0095 & 2.5017 & 0.00000027 \\
\hline 12 & -0.0061 & 4.3137 & 0.00000006 \\
\hline 13 & -0.014 & -1.5312 & 0.00000014 \\
\hline 14 & 0.0051 & -0.6632 & 0.00000018 \\
\hline 15 & -0.0102 & 1.6083 & 0.00000032 \\
\hline 16 & -0.0023 & -1.5139 & 0.00000031 \\
\hline 17 & -0.0066 & 6.0243 & -0.00000011 \\
\hline 18 & -0.0037 & 5.1447 & 0.00000011 \\
\hline 19 & -0.014 & 1.4385 & 0.00000041 \\
\hline 20 & -0.0032 & 4.1677 & 0.00000021 \\
\hline 21 & 0.0061 & 4.6595 & 0.00000012 \\
\hline 22 & -0.0127 & 6.8996 & 0.00000022 \\
\hline $\mathrm{X}$ & 0.0068 & 4.4555 & -0.00000053 \\
\hline $\mathrm{Y}$ & -0.004 & 4.9267 & 0.00000061 \\
\hline & & & \\
\hline
\end{tabular}

Table 7.12. Coefficients for chromosome models for patient A. 


\begin{tabular}{|r|r|r|r|}
\hline chr & \multicolumn{1}{|c|}{$\boldsymbol{\beta 1}$} & \multicolumn{1}{c|}{$\boldsymbol{\beta} \mathbf{2}$} & \multicolumn{1}{c|}{$\boldsymbol{\beta 3}$} \\
\hline 1 & -0.0029 & -0.7871 & -0.00000014 \\
\hline 2 & 0.0081 & -2.3130 & 0.00000051 \\
\hline 3 & 0.0108 & 5.0479 & 0.00000038 \\
\hline 4 & 0.0115 & 5.2580 & -0.00000048 \\
\hline 5 & -0.0128 & -5.3196 & 0.00000018 \\
\hline 6 & 0.0042 & 3.9586 & 0.00000096 \\
\hline 7 & 0.0150 & 2.7207 & -0.00000069 \\
\hline 8 & 0.0083 & 7.9148 & 0.00000024 \\
\hline 9 & 0.0143 & -7.0051 & 0.00000015 \\
\hline 10 & 0.0009 & 5.6727 & -0.00000033 \\
\hline 11 & 0.0168 & 3.8659 & -0.00000001 \\
\hline 12 & 0.0031 & 3.1030 & 0.00000029 \\
\hline 13 & -0.0228 & -2.5005 & 0.00000088 \\
\hline 14 & 0.0056 & 0.5399 & 0.00000067 \\
\hline 15 & -0.0089 & 2.9604 & 0.00000046 \\
\hline 16 & 0.0018 & -0.4690 & 0.00000035 \\
\hline 17 & -0.0136 & 6.4899 & -0.00000038 \\
\hline 18 & 0.0059 & 6.4388 & 0.00000000 \\
\hline 19 & -0.0180 & 1.6259 & -0.00000027 \\
\hline 20 & -0.0051 & 3.3770 & 0.00000029 \\
\hline 21 & 0.0040 & 5.0753 & -0.00000072 \\
\hline 22 & -0.0105 & 6.8326 & 0.00000019 \\
\hline $\mathrm{X}$ & 0.0163 & 4.8320 & 0.00000026 \\
\hline $\mathrm{Y}$ & 0.0053 & 4.8783 & 0.00000132 \\
\hline
\end{tabular}

Table 7.13. Coefficients for chromosome models for patient B. 


\begin{tabular}{|r|r|r|r|}
\hline $\mathbf{c h r}$ & \multicolumn{1}{|c|}{$\boldsymbol{\beta 1}$} & $\boldsymbol{\beta} \mathbf{r}$ & \multicolumn{1}{c|}{$\boldsymbol{\beta 3}$} \\
\hline 1 & -0.0072 & -0.2514 & 0.00000071 \\
\hline 2 & 0.0110 & -1.1303 & -0.00000086 \\
\hline 3 & 0.0065 & 4.7241 & 0.00000039 \\
\hline 4 & 0.0077 & 4.2140 & -0.00000051 \\
\hline 5 & -0.0145 & -7.4037 & 0.00000050 \\
\hline 6 & 0.0047 & 4.9642 & -0.00000016 \\
\hline 7 & 0.0101 & 2.7127 & 0.00000057 \\
\hline 8 & 0.0136 & 7.7381 & 0.00000018 \\
\hline 9 & 0.0122 & -5.4405 & -0.00000064 \\
\hline 10 & -0.0046 & 7.0865 & 0.00000008 \\
\hline 11 & 0.0186 & 3.1069 & -0.00000016 \\
\hline 12 & -0.0078 & 4.4835 & 0.00000026 \\
\hline 13 & -0.0146 & -2.2882 & 0.00000107 \\
\hline 14 & 0.0047 & -0.2342 & 0.00000085 \\
\hline 15 & -0.0132 & 1.0773 & -0.00000027 \\
\hline 16 & -0.0039 & -0.9811 & 0.00000116 \\
\hline 17 & 0.0025 & 6.0216 & -0.00000056 \\
\hline 18 & 0.0048 & 6.2068 & -0.00000063 \\
\hline 19 & -0.0237 & 0.0305 & 0.00000055 \\
\hline 20 & 0.0041 & 4.9333 & -0.00000006 \\
\hline 21 & 0.0017 & 5.8057 & -0.00000013 \\
\hline 22 & -0.0162 & 6.9951 & -0.00000029 \\
\hline $\mathrm{X}$ & 0.0050 & 5.2612 & -0.00000036 \\
\hline $\mathrm{Y}$ & -0.0020 & 6.1814 & 0.00000152 \\
\hline
\end{tabular}

Table 7.14. Coefficients for chromosome models for patient C. 


\begin{tabular}{|r|r|r|r|}
\hline chr & \multicolumn{1}{|c|}{$\boldsymbol{\beta 1}$} & \multicolumn{1}{c|}{$\boldsymbol{\beta} \mathbf{2}$} & \multicolumn{1}{c|}{$\boldsymbol{\beta 3}$} \\
\hline 1 & -0.0076 & -0.7604 & -0.00000076 \\
\hline 2 & 0.0067 & -0.7116 & 0.00000096 \\
\hline 3 & 0.0097 & 5.7364 & -0.00000048 \\
\hline 4 & 0.0139 & 4.0246 & 0.00000114 \\
\hline 5 & -0.0202 & -4.9885 & -0.00000002 \\
\hline 6 & 0.0147 & 4.4609 & -0.00000022 \\
\hline 7 & 0.0042 & 2.6177 & 0.00000107 \\
\hline 8 & 0.0128 & 8.7581 & 0.00000091 \\
\hline 9 & 0.0192 & -5.4743 & 0.00000070 \\
\hline 10 & 0.0022 & 6.3766 & 0.00000037 \\
\hline 11 & 0.0194 & 1.7699 & 0.00000021 \\
\hline 12 & 0.0033 & 3.6173 & 0.00000102 \\
\hline 13 & -0.0120 & -2.0102 & 0.00000015 \\
\hline 14 & 0.0064 & 0.7180 & 0.00000026 \\
\hline 15 & -0.0081 & 2.5469 & -0.00000058 \\
\hline 16 & 0.0023 & -2.9014 & 0.00000071 \\
\hline 17 & -0.0021 & 5.4218 & 0.00000084 \\
\hline 18 & -0.0080 & 4.2675 & 0.00000001 \\
\hline 19 & -0.0200 & 2.3603 & 0.00000068 \\
\hline 20 & -0.0035 & 3.4451 & -0.00000044 \\
\hline 21 & 0.0142 & 4.8766 & -0.00000044 \\
\hline 22 & -0.0171 & 7.1129 & 0.00000082 \\
\hline $\mathrm{X}$ & 0.0148 & 4.2595 & -0.00000152 \\
\hline $\mathrm{Y}$ & -0.0040 & 5.3937 & 0.00000005 \\
\hline
\end{tabular}

Table 7.15. Coefficients for chromosome models for patient D. 


\begin{tabular}{|r|r|r|r|}
\hline $\mathbf{c h r}$ & \multicolumn{1}{|c|}{$\boldsymbol{\beta 1}$} & \multicolumn{1}{c|}{$\boldsymbol{\beta 2}$} & $\boldsymbol{\beta} \mathbf{3}$ \\
\hline 1 & 0.0019 & 0.4059 & 0.00000012 \\
\hline 2 & 0.0140 & -2.2165 & 0.00000066 \\
\hline 3 & 0.0023 & 3.8831 & 0.00000013 \\
\hline 4 & 0.0081 & 6.7147 & 0.00000009 \\
\hline 5 & -0.0197 & -5.9144 & 0.00000043 \\
\hline 6 & 0.0138 & 4.0495 & 0.00000075 \\
\hline 7 & 0.0187 & 2.9437 & -0.00000072 \\
\hline 8 & 0.0127 & 8.1505 & 0.00000027 \\
\hline 9 & 0.0087 & -5.4002 & 0.00000033 \\
\hline 10 & 0.0033 & 6.2483 & 0.00000059 \\
\hline 11 & 0.0191 & 1.8344 & 0.00000074 \\
\hline 12 & -0.0079 & 3.8368 & 0.00000102 \\
\hline 13 & -0.0147 & -1.0476 & 0.00000054 \\
\hline 14 & 0.0080 & -0.7254 & -0.00000023 \\
\hline 15 & -0.0154 & 2.5250 & -0.00000002 \\
\hline 16 & 0.0073 & -0.6750 & 0.00000067 \\
\hline 17 & -0.0016 & 6.0702 & 0.00000011 \\
\hline 18 & -0.0014 & 6.4145 & -0.00000033 \\
\hline 19 & -0.0041 & 0.9393 & 0.00000135 \\
\hline 20 & -0.0042 & 3.8852 & 0.00000075 \\
\hline 21 & 0.0148 & 5.5249 & 0.00000020 \\
\hline 22 & -0.0178 & 7.6082 & -0.00000075 \\
\hline $\mathrm{X}$ & 0.0144 & 5.5756 & -0.00000133 \\
\hline $\mathrm{Y}$ & 0.0007 & 4.0187 & 0.00000059 \\
\hline
\end{tabular}

Table 7.16. Coefficients for chromosome models for patient E. 


\begin{tabular}{|r|r|r|r|}
\hline chr & \multicolumn{1}{|c|}{$\boldsymbol{\beta 1}$} & \multicolumn{1}{c|}{$\boldsymbol{\beta} \mathbf{2}$} & \multicolumn{1}{c|}{$\boldsymbol{\beta 3}$} \\
\hline 1 & 0.0013 & -1.8080 & -0.00000024 \\
\hline 2 & 0.0140 & -0.8890 & -0.00000031 \\
\hline 3 & 0.0025 & 3.7933 & -0.00000064 \\
\hline 4 & 0.0101 & 6.0363 & 0.00000093 \\
\hline 5 & -0.0157 & -5.1467 & 0.00000096 \\
\hline 6 & 0.0131 & 5.4381 & 0.00000094 \\
\hline 7 & 0.0067 & 3.8777 & 0.00000093 \\
\hline 8 & 0.0183 & 6.8689 & -0.00000070 \\
\hline 9 & 0.0219 & -5.3636 & 0.00000030 \\
\hline 10 & -0.0082 & 6.2296 & 0.00000044 \\
\hline 11 & 0.0021 & 3.8518 & 0.00000038 \\
\hline 12 & -0.0123 & 3.1948 & 0.00000086 \\
\hline 13 & -0.0161 & -0.6092 & -0.00000002 \\
\hline 14 & 0.0145 & -0.4893 & 0.00000091 \\
\hline 15 & -0.0103 & 2.5162 & -0.00000055 \\
\hline 16 & 0.0036 & -2.8785 & -0.00000054 \\
\hline 17 & -0.0074 & 6.0751 & 0.00000016 \\
\hline 18 & 0.0011 & 4.9096 & 0.00000031 \\
\hline 19 & -0.0068 & 1.4684 & 0.00000077 \\
\hline 20 & 0.0053 & 2.9407 & 0.00000085 \\
\hline 21 & 0.0109 & 5.0740 & -0.00000079 \\
\hline 22 & -0.0151 & 7.9485 & -0.00000032 \\
\hline $\mathrm{X}$ & 0.0130 & 3.6375 & 0.00000014 \\
\hline $\mathrm{Y}$ & 0.0007 & 3.9946 & -0.00000030 \\
\hline
\end{tabular}

Table 7.17. Coefficients for chromosome models for patient $\mathrm{F}$. 


\begin{tabular}{|r|r|r|r|}
\hline chr & \multicolumn{1}{|c|}{$\boldsymbol{\beta 1}$} & \multicolumn{1}{c|}{$\boldsymbol{\beta} \mathbf{2}$} & \multicolumn{1}{c|}{$\boldsymbol{\beta 3}$} \\
\hline 1 & -0.0013 & -0.0143 & 0.00000090 \\
\hline 2 & 0.0104 & -2.0696 & -0.00000036 \\
\hline 3 & 0.0096 & 3.3256 & 0.00000011 \\
\hline 4 & 0.0070 & 6.3824 & 0.00000013 \\
\hline 5 & -0.0026 & -5.3594 & -0.00000045 \\
\hline 6 & 0.0137 & 5.4012 & 0.00000101 \\
\hline 7 & 0.0051 & 2.8205 & 0.00000025 \\
\hline 8 & 0.0151 & 8.2805 & -0.00000058 \\
\hline 9 & 0.0165 & -5.7032 & 0.00000071 \\
\hline 10 & 0.0032 & 6.6396 & 0.00000035 \\
\hline 11 & 0.0055 & 3.3530 & 0.00000095 \\
\hline 12 & 0.0035 & 5.5225 & -0.00000042 \\
\hline 13 & -0.0128 & -0.8615 & 0.00000077 \\
\hline 14 & 0.0095 & -1.3463 & -0.00000080 \\
\hline 15 & -0.0031 & 0.7591 & 0.00000131 \\
\hline 16 & 0.0049 & -0.7272 & 0.00000082 \\
\hline 17 & 0.0031 & 5.9467 & 0.00000026 \\
\hline 18 & 0.0026 & 4.4366 & 0.00000110 \\
\hline 19 & -0.0190 & 2.3530 & -0.00000035 \\
\hline 20 & 0.0047 & 4.5874 & -0.00000040 \\
\hline 21 & 0.0160 & 4.3033 & -0.00000043 \\
\hline 22 & -0.0190 & 7.7892 & 0.00000057 \\
\hline $\mathrm{X}$ & 0.0126 & 3.1060 & -0.00000125 \\
\hline $\mathrm{Y}$ & 0.0007 & 5.4127 & 0.00000069 \\
\hline
\end{tabular}

Table 7.18. Coefficients for chromosome models for patient $G$ 


\begin{tabular}{|r|r|r|r|}
\hline $\mathbf{c h r}$ & \multicolumn{1}{|c|}{$\boldsymbol{\beta 1}$} & $\boldsymbol{\beta} \mathbf{r}$ & $\boldsymbol{\beta} \mathbf{\beta 3}$ \\
\hline 1 & 0.0032 & -0.3078 & -0.00000059 \\
\hline 2 & 0.0012 & -0.1699 & -0.00000048 \\
\hline 3 & 0.0069 & 5.6587 & 0.00000034 \\
\hline 4 & 0.0071 & 5.6634 & -0.00000021 \\
\hline 5 & -0.0095 & -5.7618 & -0.00000061 \\
\hline 6 & 0.0096 & 5.0768 & 0.00000005 \\
\hline 7 & 0.0091 & 4.3699 & 0.00000022 \\
\hline 8 & 0.0166 & 7.2473 & 0.00000020 \\
\hline 9 & 0.0219 & -6.1187 & 0.00000011 \\
\hline 10 & 0.0018 & 6.7467 & 0.00000037 \\
\hline 11 & 0.0184 & 2.8218 & -0.00000042 \\
\hline 12 & 0.0037 & 4.5330 & 0.00000079 \\
\hline 13 & -0.0176 & -1.8377 & 0.00000106 \\
\hline 14 & 0.0113 & 0.1349 & -0.00000049 \\
\hline 15 & -0.0192 & 1.6409 & 0.00000095 \\
\hline 16 & -0.0046 & -0.7617 & -0.00000046 \\
\hline 17 & 0.0013 & 6.2843 & 0.00000011 \\
\hline 18 & -0.0074 & 4.9845 & 0.00000013 \\
\hline 19 & -0.0075 & 0.7691 & 0.00000034 \\
\hline 20 & -0.0062 & 3.9471 & 0.00000032 \\
\hline 21 & 0.0034 & 4.7239 & -0.00000039 \\
\hline 22 & -0.0194 & 6.4096 & 0.00000041 \\
\hline $\mathrm{X}$ & 0.0141 & 3.5085 & -0.00000091 \\
\hline $\mathrm{Y}$ & 0.0013 & 5.1003 & 0.00000093 \\
\hline
\end{tabular}

Table 7.19. Coefficients for chromosome models for patient $\mathrm{H}$ 


\subsubsection{Comparison of performance of our model versus other algorithms}

In order to evaluate our framework better, we compared our proposed framework with three state-of-the-art algorithms which are Support Vector Machines using Sequential Minimal Optimization, Gaussian Process regression model and Radial Basis Function Network. The following paragraphs briefly explain them in more detail.

SVMs (Support Vector Machines) is a well known technique widely used for classification and regression. It is one of the most popular state-of-the-art algorithms which can be utilized to provide a model for linear and non-linear problems. Mathematically speaking, the SVM model can be interpreted as solving the optimization problem below:

$$
\min _{w, b, \varepsilon} \frac{1}{2} w^{T} w+C \sum_{i=1}^{l} \xi_{i}
$$

Subject to

$y_{i}\left(w^{T} \phi\left(x_{i}\right)+b\right) \geq 1-\xi_{i}$,

$\xi_{i} \geq 0$

Where ${ }^{x_{i}}$ : training vectors.

$w$ : weight vectors.

$\phi\left(x_{i}\right)$ : kernel function

b: the bias.

$\xi$ : parameter to handle over-fitting.

Here we utilized one major implementation of SVM, which is the sequential minimal optimization (SMO) implementation to compare to the proposed framework. The 
performance is given in figure 7.17. It shows that it performs worse than the proposed algorithm. In addition, there is a lot of variance in the performance of the SVM-based solution. The possible reason is the over fitting problem. More details can be found in [119].

Gaussian Processes for Regression

The Gaussian process for regression is well known model for machine-learning. It is a generalized form of the Gaussian probability distribution. Similar to the correspondent distribution, the Gaussian process for regression is determined by the covariance and mean function. The function here $\mathrm{f}(\mathrm{x})$ comes from just one sample from the actual distribution. More details on this method can be found in [120].

\section{Radial Basis Function}

It follows an artificial neural network approach which employs functions that are based on a radial basis. Radial basis functions based on neuron arguments are the output of this network. For a given input $\mathrm{x}, \mathbf{x} \in \mathbb{R}^{n}$, the algorithm produces a scalar function, $\varphi: \mathbb{R}^{n} \rightarrow \mathbb{R}$, given by:

$\varphi(\mathbf{x})=\sum_{i=1}^{N} a_{i} \rho\left(\left\|\mathbf{x}-\mathbf{c}_{i}\right\|\right)$ 
Where

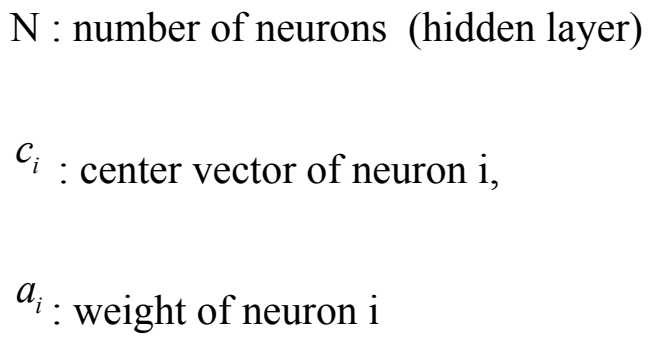

For more details on this algorithm, we can refer to [121].

The comparison results are shown in Table 7.20 and Figure 7.17. For this, we compared the correlation coefficients achieved by each different algorithm and verified that our selected model had a better performance and stability across all chromosomes. We can observe that the Radial Basis Function Network shows the highest variance. This is very dangerous in the medical domain as the predicted values show high variability. The other two algorithms have a better performance when compared to the Radial Basis Function Network; however, they also still lower performance than our selected model and higher variance. Our model, on the other hand, is stable across all chromosomes showing more accurate predictions. 


\begin{tabular}{|r|r|r|r|r|}
\hline Chr & \multicolumn{1}{l|}{$\begin{array}{l}\text { Linear } \\
\text { Regression }\end{array}$} & $\begin{array}{l}\text { Radial Basis } \\
\text { Function } \\
\text { Network }\end{array}$ & $\begin{array}{l}\text { Sequential } \\
\text { Minimal } \\
\text { Optimization }\end{array}$ & GaussianProcess \\
\hline $\mathbf{1}$ & 0.9412 & 0.4533 & 0.9389 & 0.9356 \\
\hline $\mathbf{2}$ & 0.9999 & 0.8452 & 0.9253 & 0.9235 \\
\hline $\mathbf{3}$ & 0.9994 & 0.3159 & 0.9993 & 0.9235 \\
\hline $\mathbf{4}$ & 0.9769 & 0.8627 & 0.9718 & 0.9741 \\
\hline $\mathbf{5}$ & 0.9475 & 0.4144 & 0.9448 & 0.9373 \\
\hline $\mathbf{6}$ & 0.9998 & 0.0806 & 0.9985 & 0.9909 \\
\hline $\mathbf{7}$ & 0.9826 & 0.5022 & 0.9383 & 0.9956 \\
\hline $\mathbf{8}$ & 0.9972 & 0.0616 & 0.9972 & 0.9235 \\
\hline $\mathbf{9}$ & 0.9403 & 0.0112 & 0.8055 & 0.9307 \\
\hline $\mathbf{1 0}$ & 0.9896 & 0.1312 & 0.9819 & 0.9827 \\
\hline $\mathbf{1 1}$ & 0.9565 & 0.4605 & 0.9192 & 0.9436 \\
\hline $\mathbf{1 2}$ & 0.9946 & 0.9368 & 0.9946 & 0.7254 \\
\hline $\mathbf{1 3}$ & 0.9999 & 0.4473 & 0.8715 & 0.9657 \\
\hline $\mathbf{1 4}$ & & 0.1127 & 1 & 0.9973 \\
\hline $\mathbf{1 5}$ & 0.9995 & 0.957 & 0.9993 & 0.9762 \\
\hline $\mathbf{1 6}$ & 0.9609 & 0.0206 & 0.9609 & 0.8614 \\
\hline $\mathbf{1 7}$ & 0.9987 & 0.8142 & 0.9982 & 0.9142 \\
\hline $\mathbf{1 8}$ & 0.9914 & 0.969 & 0.9875 & 0.9654 \\
\hline $\mathbf{1 9}$ & 0.9885 & 0.9045 & 0.9829 & 0.9773 \\
\hline $\mathbf{2 0}$ & 0.9702 & 0.2325 & 0.9625 & 0.9611 \\
\hline $\mathbf{2 1}$ & 0.9959 & 0.3255 & 0.9958 & 0.9992 \\
\hline $\mathbf{2 2}$ & 0.9786 & 0.1466 & 0.7901 & 0.9334 \\
\hline $\mathbf{X}$ & 0.9922 & 0.0569 & 0.9892 & 0.9728 \\
\hline $\mathbf{Y}$ & 0.9992 & 0.8394 & 0.9986 & 0.9728 \\
\hline & & & & \\
\hline
\end{tabular}

Table 7.20. Comparison table for correlation coefficients for our model versus others. 


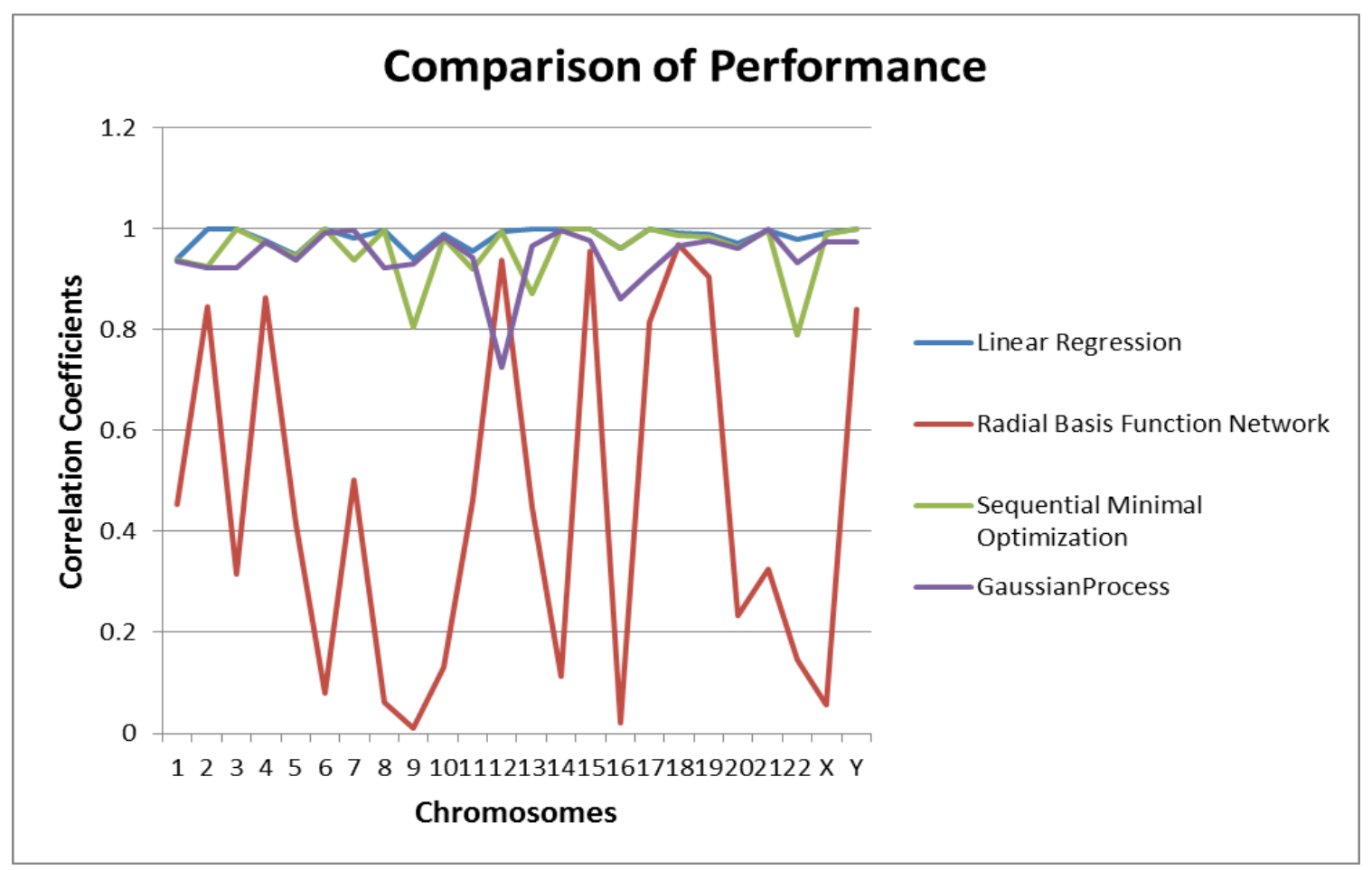

Figure 7.17 Comparison of performance of our model versus others.

\subsubsection{Concluding Remarks}

The identification and classification of mutations in patients are centric topics in today's cancer research. In this study, we first defined the problem of predicting the number of mutations on a chromosome for a certain patient, which is a fundamental problem in both research and clinical fields. Next, this problem is modeled mathematically as a linear regression problem. After applying a thorough analysis on each chromosome of each patient, we have the following observations:

1. As shown in the detailed statistical analysis in terms of error, the $95 \%$ confidence interval of each of the error measurement metrics are computed. For example, the correlation coefficient, which indicates the degree of matching of the proposed 
framework with real data, is $0.9816 \pm 0.009$ with $95 \%$ confidence interval. In addition, our collaborator from medical domain thinks that the proposed framework achieves very good performance and opens new research opportunities for bioinformatics researchers and clinical doctors.

2. The proposed framework is relatively stable across patients. By analyzing errors of each patient, it can be seen that the errors are close to each other across different patients. Since patients are selected randomly, this observation gives us the confidence that the proposed framework is relatively stable and robust. It can be applied on new data collected from new patients.

3. In terms of the coefficients learned from different chromosomes on different patients, no significant global patterns are identified. This indicates that each individual patient has unique characteristic and it is hard to generalize a simple rule based strategy for all patients. On the other hand, the proposed framework which trains the linear regression model can adapt to each patient relatively well by representing the different characteristics of patients in the numerical weights gained from the model. This explains the reason why our framework achieves relatively stable performance across different patients. In addition, an observation is that the coefficient for the ChrToGenomeRatio feature is relatively small in all models, which indicate its' contribution is limited. More features are going to be added to enhance the model further in the future.

As we train the model with more longitudinal data, the estimation error should decrease. An example graph shown in the next Figure illustrates this statement. Additionally, as we collect more data points, in the future, we open the possibility to experiment other machine-learning algorithms as well. 


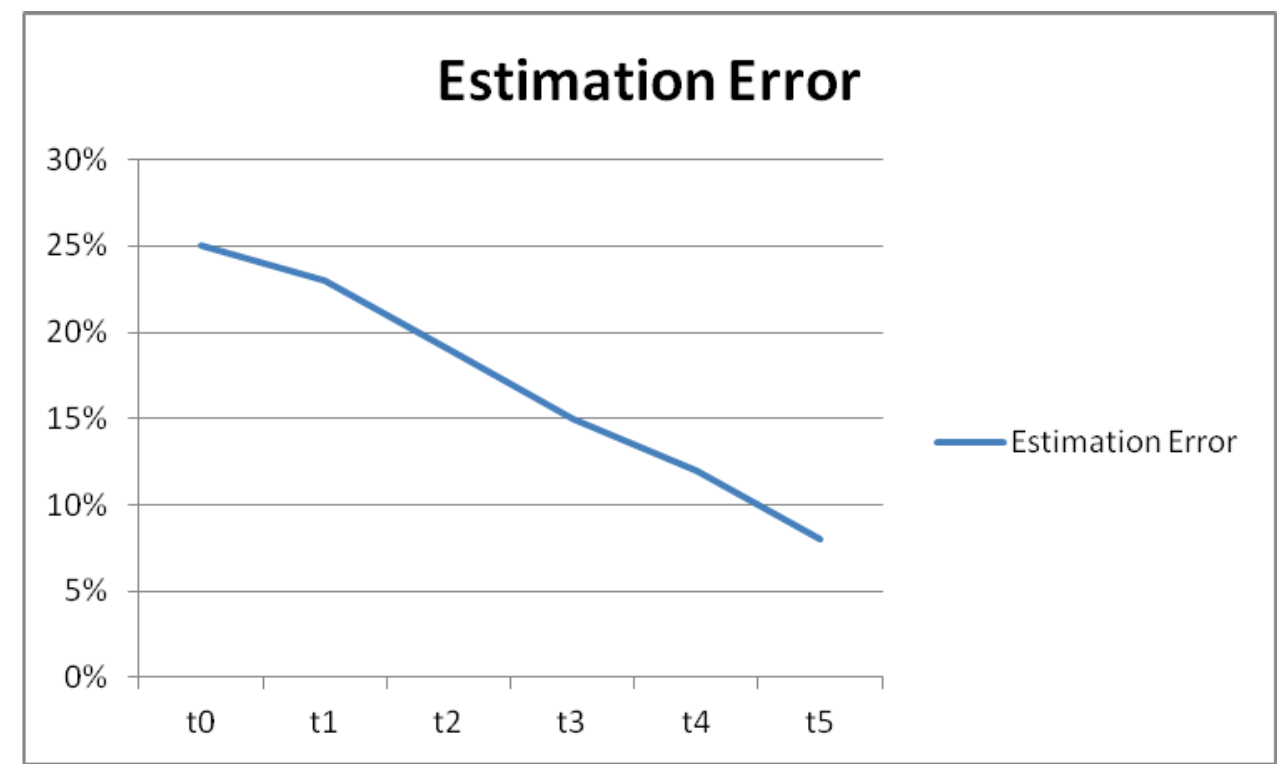

Figure 7.18 Potential estimation error graph as more data is obtained.

\subsection{Prediction of location of mutations.}

Table 7.2 refers to the locations of mutations, both observed and predicted, for all chromosomes in patient UPN 933124. Column two and three show the nucleotide positions of the mutations. Column four shows the name of the gene, if present in the region, in the case of observed mutations. Similarly, column five shows the name of the gene if present in the region corresponding to the predicted mutations. Not surprisingly, most of the areas containing the mutations (both observed and predicted) lie in the socalled intergenic regions of the genome. It is worth noting that the areas of the genome transcriptionally active are reported to be just $1 \%$ of the whole genome [122]. Close to $99 \%$ of the human genome does not code for proteins, and correspond to areas with either other known functionalities as regulation of gene expression (enhancers, promoters, inhibitors) or that do not have a defined function yet. 


\begin{tabular}{|c|c|c|c|c|}
\hline Chr & $\begin{array}{c}\text { Observed } \\
\text { Location }\end{array}$ & $\begin{array}{c}\text { Location of the } \\
\text { predicted mutation }\end{array}$ & $\begin{array}{c}\text { Gene or genetic } \\
\text { region observed }\end{array}$ & $\begin{array}{c}\text { Gene or genetic } \\
\text { region predicted }\end{array}$ \\
\hline 1 & 107357719 & 107358018 & INTERGENIC & INTERGENIC \\
\hline 1 & 179307551 & 179309065 & INTERGENIC & INTERGENIC \\
\hline 1 & 218018901 & 218020628 & INTERGENIC & INTERGENIC \\
\hline 2 & 184186967 & 184186891 & INTERGENIC & INTERGENIC \\
\hline 2 & 213886052 & 213884438 & SPAG16 & SPAG16 \\
\hline 3 & 21310708 & 21307866 & INTERGENIC & INTERGENIC \\
\hline 3 & 43135962 & 43138381 & INTERGENIC & INTERGENIC \\
\hline 4 & 26362673 & 26362567 & TBC1D19 & TBC1D19 \\
\hline 5 & 22595465 & 22595335 & CDH12 & CDH12 \\
\hline 5 & 93857376 & 93859895 & INTERGENIC & INTERGENIC \\
\hline 6 & 40467367 & 40464779 & LRFN2 & INTERGENIC \\
\hline 6 & 95631430 & 95629374 & INTERGENIC & INTERGENIC \\
\hline 7 & 85476859 & 85474442 & INTERGENIC & INTERGENIC \\
\hline 7 & 120917535 & 120914752 & LOC392979 & LOC392979 \\
\hline 8 & 25022615 & 25022103 & INTERGENIC & INTERGENIC \\
\hline 8 & 34167561 & 34165529 & INTERGENIC & INTERGENIC \\
\hline 8 & 35476742 & 35478696 & INTERGENIC & INTERGENIC \\
\hline 8 & 51256369 & 51253560 & SNTG1 & SNTG1 \\
\hline 9 & 36887024 & 36889733 & PAX5 & PAX5 \\
\hline 9 & 137816463 & 137818783 & KCNT1 & KCNT1 \\
\hline 11 & 23583427 & 23584671 & INTERGENIC & INTERGENIC \\
\hline 11 & 40183906 & 40182763 & LRRC4C & LRRC4C \\
\hline 12 & 9702101 & 9703657 & LOC374443 & INTERGENIC \\
\hline 12 & 22283439 & 22281122 & ST8SIA1 & ST8SIA1 \\
\hline 13 & 60889651 & 60891444 & INTERGENIC & INTERGENIC \\
\hline 13 & 92466026 & 92467721 & INTERGENIC & INTERGENIC \\
\hline 14 & 47961450 & 47963585 & INTERGENIC & INTERGENIC \\
\hline
\end{tabular}




\begin{tabular}{|c|c|c|c|c|}
\hline 14 & 82608644 & 82607776 & INTERGENIC & INTERGENIC \\
\hline 16 & 7941529 & 7943725 & INTERGENIC & INTERGENIC \\
\hline 16 & 58050635 & 58052689 & INTERGENIC & INTERGENIC \\
\hline 17 & 67861248 & 67859454 & INTERGENIC & INTERGENIC \\
\hline 20 & 17261178 & 17262629 & PCSK2 & PCSK2 \\
\hline 20 & 58861532 & 58863711 & INTERGENIC & INTERGENIC \\
\hline 21 & 23370033 & 23372348 & INTERGENIC & LOC100130310 \\
\hline $\mathrm{X}$ & 86570002 & 86570879 & INTERGENIC & INTERGENIC \\
\hline
\end{tabular}

Table 7.21. Location of mutations, both observed and predicted, for patient UPN 933124.

\subsection{Accuracy discussion}

Table 7.3 shows the results of the analysis for the patient UPN 933124. It presents the total number of mutations per genome, number of matches, mismatches, and accuracy of the prediction. When the prediction lies within the same region of an observed mutation, it constitutes a match. Conversely, if the location for the prediction does not correspond to the location of an observed mutation, or if the area of the observed mutation is not predicted at all, it is considered a mismatch.

We emphasize that the location of the prediction does not have to match the precise location of the observed mutation. Falling within the same gene boundaries is sufficient to classify it as a match. When the location of the mutation falls in an intergenic region (or a region devoid of a known functional activity) one criterion we use to classify it as a match, is that the prediction lies within less than 3000 nucleotides of the observed one on both directions. Although the distance of 3000 nucleotides may look arbitrary, to the best of our knowledge it is a reasonable assumption when there is no knowledge as to the functional features determined in such an intergenic region. 


\begin{tabular}{|l|l|}
\hline Matches & 32 \\
\hline Mismatches & 3 \\
\hline Total Mutations & 35 \\
\hline Relative Accuracy & $91 \%$ \\
\hline Absolute Accuracy & $76 \%$ \\
\hline
\end{tabular}

Table 7.22. Summary of results for patient UPN 933124.

With these criteria in mind, we came up with two accuracies. The first one is the "Relative Accuracy" which measures how many matches we had out of the predicted mutations we accounted for. For instance, in the case of the patient UPN 933124, we found out that even though the number of mutations at time of relapse was 42 , we only predicted 35 mutations with 32 of them classified as matches. As a result, the accuracy was $91 \%(32 / 35)$. This accuracy is called "Relative Accuracy" because we are not considering the initial error given by the fact that the number of predicted mutations (35) was lower than the actual ones (42).

The "Absolute Accuracy" incorporates this initial prediction error. Thus, its value is less than the "Relative Accuracy". For instance, for this patient, the initial accuracy for the number of predicted mutations was $83 \%$ and the "Relative Accuracy" was $91 \%$, meaning that we really could only predict for this patient $83 \%(91 \%)$ of the total number of mutations correctly, giving us an accuracy of $76 \%$.

We carried out similar analysis for the seven remaining individuals. The accuracies are given as ranges with lower and upper values. This is explained by the fact that chromosomes models behave differently within the same individual, as well as, across patients. The verification of the predicted number of mutations versus the actual number 
of mutations gave us an accuracy value within a range of 75 to $84 \%$. Likewise, the verification of predicted locations of mutations versus actual locations of mutations resulted in an accuracy value within the range of 69 to $88 \%$. 


\section{CHAPTER 8}

\section{CONCLUSION}

\subsection{Research summary}

The efforts and techniques used in this thesis represent the best methods we know to date in identifying meaningful mutational changes in leukemias that can help predict the following: 1.) responses to therapy, 2.) patients at increased risk of developing recurrences or progression of disease before and after treatment, 3.) driver mutations, 4.) passenger mutations, 5.) and evaluation of new potential druggable targets.

The $\mathrm{n}$ (\# of patients) of 8 used in this study is actually a fundamental strength of the proposed study for the following reasons. Most research examining mutational spectra within cancers rely solely on snapshots, i.e. the presence or absence of mutations in tumors at one discrete time point. Though this information is valuable, it tells us nothing regarding the evolution of the disease. In particular, several high profile papers in the last 2-3 years have taken advantage of the TCGA database to mine solid tumors for mutations in these snapshots - work out of robust genome sequencing groups including from Washington University, St Louis (Science, 2012) and Harvard. All of these papers appear in very high end journals but use data from only one time point. A recent paper in Nature (2013) offered similar comparisons of such samples. It is more straightforward to have n's approaching 100-200+ patients for these less complex situations.

The gold standard really should be to get genomic mutational data for tumors from patients across their disease course over multiple time points. This longitudinal 
information represents the most valuable and relevant data in truly determining how to cure patients of disease. A paper in the very important journal Cell from early 2013 evaluated CLL patient tumor mutational spectra [123]. This work came out of a collaboration between the BROAD Institute of MIT and Harvard and Dana Farber Cancer Center, highly renowned for their leukemia patient volume. They had only snapshots of 140 or so patients for which they were able to identify mutation frequency. However, as the reviewers and authors acknowledge, they had a more valuable data set - 18 patients for which they had longitudinal mutational spectra from 2 time points - before and after chemotherapy. Only sequencing of coding areas were used in this effort. With 18 patients, they were able to make conclusions regarding the evolution of the CLL disease course, helping oncologists further understand the disease process to improve the lives of the patients. All agreed that this 18 patient data set was in many ways more valuable than the 140 patient snapshot mutational database. Additionally, NCI has recently made it a priority to better understand unique findings in smaller data series if the samples are difficult to come by. Longitudinal mutational data is very difficult to come by and perhaps offers the most important clues to disease processes.

In our work, we have been able to collect 8 valuable longitudinal mutational data from leukemia patients before and after multiple therapies. This collection may represent one of the largest series compiled in a robust manner. Our sequencing data is of the entire genome, unlike the coding only analysis from the Cell paper. Finally, our data approximates locations of mutational hot spots, which is very important because many changes in leukemia tumor DNA represent large frame shift alterations, deletions, insertions, or translocations. 


\subsection{Final statistical remarks}

We analyze the mutations in the longitudinal dimension at the chromosome level. From this perspective, one patient actually provides 24 samples. Therefore, instead of 8 samples, we actually test our algorithm using $24 \times 8=192$ samples. This amount of data is significant in terms of regular statistical and data mining tasks. Compared with peer studies in the cancer research domain as mentioned by our collaborator in biomedical field, our number of patients as well as the measurements of mutations at multiple time shots for each patient are more significant than the recent published papers in Nature and Science. Even though the data samples are actually enough for significance, we still draw the conclusion prudently. In our statistical analysis of errors, the routing approach of estimating the confidence interval is using the normal distribution assumption. If that assumption is used here, the variation of our estimation is even smaller. However, given the current sample size, we still use the student-T distribution assumption to estimate errors. By using this, we draw a conservative conclusion. Given this observation, we would expect the performance of our algorithm on new data sets would be better.

As the cost of performing the whole genome sequencing is relatively expensive nowadays, it is infeasible to carry out the whole genome sequencing for the cancer patient routinely. On the other hand, the information of mutations in chromosomes at the molecular level gives precious information about patients and can guide clinical doctors in practice. The gap exists here should be addressed in order to improve the quality of treatments for patients. In addition, each patient has his/her individual characteristics which need to be considered. The proposed work provides a study from a relatively novel angle. First and foremost, a linear regression model is fit using three input features, which 
are "RatioStdMean", "RatioGivenChr" and "ChrToGenomRation". Since the number of patients' visitings to hospitals is limited, the number of samples from each patient is sparse. Our model can take care of this issue since the model can be fit using features from as few as two time points. In addition, a model is built on each chromosome for each patient, this model adapts to each patient's specialty and therefore is relatively robust. From the clinical point of view, the predictions of mutations can be meaningful for clinical doctors from the following two perspectives. First, the mutation frequency and patterns of the chromosomes of patients are useful information for medical doctors to make decision on the effectiveness of treatment and adjust treating strategy. Second, the mutation information can be further utilized as a factor to associate to classifying different types of cancer. A recent study in Nature actually shows that the mutation frequency has correlations with the different types of breast cancer. From this point of view, the mutation frequency predicted of each chromosome and the profile of mutation frequency predicted for each patient will offer the information for doctors to classify the cancer types and adjust treatment strategy accordingly. All these clinical significance motivates us for this research. Based on the success of applying the linear regression model in this study, we will extend the current work to a more broad problem, that is, we will try to fit a model across patients. Using this model, we can make predictions for the possibilities of mutations on a chromosome of a new patient based on the data collected from patients in our database. We believe this model, if successfully constructed, will provide more significant tools for medical practitioners. 


\subsection{Foundation for further discoveries.}

Our concepts may have tremendous impact on a number of important areas relevant to future cancer care of patients. Specifically, our ideas may:

1. Increase our ability to predict which leukemia patients will develop relapses thru treatment.

2. Help predict which high risk patients (those having received chemotherapy for other cancers, patients on immunosuppressive therapy for organ transplantation, etc.) may develop leukemia down the road.

3. Help distinguish driver versus passenger mutations in leukemia.

4. Help identify mutations that may develop during leukemogenesis that are druggable targets.

5. Ultimately help leukemia patients have longer survival.

None of these results are trivial, especially in the setting of leukemias. There is a significant advantage to our work. Our models appear to be quite robust and relevant to leukemia disease states. We have employed longitudinal data sets from actual leukemia patients that we have used to build our models and validate them. The rationale behind our efforts is also convincing. Identification of approximate areas of deletion, insertion, and other mutations will be quite valuable as we begin to realize that most mutations in malignancy may not just be driven by single nucleotide polymorphisms. 


\subsection{Application of our framework in the treatment of patients by clinical oncologists}

It is quite clear that our work will provide high impact understanding of the natural evolution of basal leukemia mutation rates and development before chemotherapy use and more importantly the state of mutations at the time of leukemia relapse. A vital question is how this data will be beneficial or relevant to a medical oncologist's practice on a day to day basis. From the initial applications of the predictive modeling of future mutation rates, we expect physician's to develop key information when they first diagnose a patient's leukemia, i.e. will there be an increase in mutation rate in the specific patient's leukemia at the time of relapse, will there be hotspots in the genome for this greater mutation rate that is associated with druggable targets, will there be at the time of relapse a completely different set of mutations (explaining why the patient's tumor relapses), etc. At the time of relapse, a medical oncologist has several decisions to make: keep the patient on the same leukemia drug but add a second drug, increase the levels of the drug in circulation, or change the drug completely. With the predictive mutation modeling, the medical oncologist can know in advance to potentially be prepared to take one of the above courses based on where the mutations may appear and in what frequency at the time of relapse. This knowledge, in the form of an algorithm of chemotherapy use, would be of great necessity in allowing medical oncologist's to optimize timing and response to patient leukemia relapses.

Finally, the greatest utility that the predictive model from this work may provide in the long run to medical oncologists and cancer specialists is a novel unbiased approach to genomically organize leukemias based on their natural evolution of mutation development. Historically, for many decades, leukemias were characterized (ALL, 
AML, CLL, CML) purely by histopathologic and cytopathologic analyses. This data was the only information used by physicians to decide how to treat patients with leukemias. This was a limited data set, however. With the identification of mutations associated with each type of leukemia, we are now incorporating this latter information into medical management practices. The use of targeted therapies including Gleevec was predicated on knowing if a leukemia possessed a bcr-abl mutation.

Despite the advances, we are still only making treatment decisions on a static evaluation of mutations at the time of diagnosis. The predictive algorithm provided in this work may open a totally new avenue by which cancer specialists can organize similar behaving leukemias into independent groups based on functional outcomes. Potentially, we would be able to characterize a leukemia as highly aggressive, aggressive, or minimally aggressive based on the prediction of mutation frequency/location at the time of relapse. Highly aggressive leukemias would be predicted to have greater mutation rates and sites at relapse, suggesting an earlier time to relapse. Minimally aggressive lesions would potentially have predicted lower rates of mutation and relapse at later time points. The power of this effort is that we would be taking into account all mutational changes (which may have distinct interactions), not just hypothesized driver mutations, to describe the ultimate output behavior of leukemias, before and after chemotherapy. A medical oncologist would be able to accurately predict how a patient with leukemia will do if he knows from the onset the likelihood of high or low mutation rates, predicting for highly or minimally aggressive disease. At the very least, with the creation of these "clades" or new ways to group leukemias based on functional outcomes, we will get a better sense of how many distinct subgroups or entities of leukemias are seen in the public. At the most, 
medical oncologists will be prepared to be more or less aggressive with changing chemotherapy regimens based on how the specific patient's leukemia is categorized. Staying ahead of the relapse conditions that promote increased mutational frequency by combining chemotherapies or changing the time of chemotherapy usage may ultimately improve progression free and overall survival, the gold standard for any cancer patient outcome.

\subsection{Some potential enhancements.}

Our framework has been built using Java technology and utilizes an external library called Weka for its prediction model. At the present time, we are using a linear regression algorithm provided by Weka. As we collect more patients' data, we will be able to perform more sophisticated prediction algorithms such as neural networks. Similarly as Weka evolves and more algorithms are included in this library, our framework will be able to extend its functionality by invoking these new features from the API. Thus, our architecture is flexible for future changes and/or enhancements. 


\section{SPECIAL RECOGNITIONS}

We would like to show our great gratitude to Puneet Iyengar, $\mathrm{MD}, \mathrm{PhD}$ for his valuable contribution to this work. He provided strong advice along the process of the writing. He aided us tremendously in performing tasks such as analysis and interpretation of data, and revising the manuscript critically. 


\section{APPENDICES}

\section{Patients Data before prediction}

HIPAA limitations are not involved here as a problem since, as mentioned earlier, we are using the published data from Ding et al publication [13].

Prediction results for predicting number of mutations at each chromosome for every patient.

On the following pages, we can see the results of our framework when predicting the number of mutations to occur at each chromosome for each patient. 
Data for Patient: $A$

\begin{tabular}{|c|c|c|}
\hline Chromosome & $\begin{array}{c}\text { Observed } \\
\text { Mutations }\end{array}$ & $\begin{array}{c}\text { Predicted } \\
\text { Mutations }\end{array}$ \\
\hline 1 & 1 & 1 \\
\hline 2 & 2 & 1 \\
\hline 5 & 1 & 1 \\
\hline 6 & 2 & 2 \\
\hline 7 & 2 & 2 \\
\hline 8 & 1 & 1 \\
\hline 10 & 1 & 1 \\
\hline 11 & 1 & 0 \\
\hline 12 & 2 & 1 \\
\hline 13 & 2 & 2 \\
\hline 15 & 2 & 1 \\
\hline 18 & 1 & 1 \\
\hline 19 & 1 & 1 \\
\hline 22 & 1 & $\mathbf{1 8}$ \\
\hline$X$ & 2 & \\
\hline Total & $\mathbf{2 2}$ & $\mathbf{8 1 . 8 2 \%}$ \\
\hline Accuracy: & & \\
\hline
\end{tabular}


Data for Patient: B

\begin{tabular}{|c|c|c|}
\hline Chromosome & $\begin{array}{c}\text { Observed } \\
\text { Mutations }\end{array}$ & $\begin{array}{c}\text { Predicted } \\
\text { Mutations }\end{array}$ \\
\hline 1 & 7 & 5 \\
\hline 2 & 10 & 7 \\
\hline 3 & 4 & 3 \\
\hline 4 & 8 & 6 \\
\hline 5 & 5 & 4 \\
\hline 6 & 7 & 5 \\
\hline 7 & 6 & 5 \\
\hline 8 & 2 & 2 \\
\hline 9 & 2 & 2 \\
\hline 10 & 7 & 5 \\
\hline 11 & 4 & 3 \\
\hline 12 & 4 & 3 \\
\hline 13 & 4 & 3 \\
\hline 14 & 3 & 2 \\
\hline 15 & 2 & 2 \\
\hline 16 & 6 & 5 \\
\hline 17 & 6 & 4 \\
\hline 18 & 3 & 2 \\
\hline 19 & 3 & 2 \\
\hline 20 & 2 & 2 \\
\hline 21 & 3 & 2 \\
\hline 22 & 2 & 1 \\
\hline$X$ & 3 & 2 \\
\hline $\mathrm{Y}$ & 2 & 2 \\
\hline Total & 105 & 79 \\
\hline Accuracy & \multicolumn{2}{|c|}{$75.24 \%$} \\
\hline
\end{tabular}




\begin{tabular}{|c|c|c|}
\hline Chromosome & $\begin{array}{c}\text { Observed } \\
\text { Mutations }\end{array}$ & $\begin{array}{c}\text { Predicted } \\
\text { Mutations }\end{array}$ \\
\hline 1 & 4 & 3 \\
\hline 2 & 2 & 2 \\
\hline 3 & 3 & 2 \\
\hline 4 & 1 & 1 \\
\hline 5 & 2 & 2 \\
\hline 6 & 2 & 2 \\
\hline 7 & 3 & 2 \\
\hline 8 & 5 & 4 \\
\hline 9 & 2 & 2 \\
\hline 11 & 3 & 2 \\
\hline 12 & 3 & 2 \\
\hline 13 & 2 & 2 \\
\hline 14 & 2 & 2 \\
\hline 16 & 3 & 1 \\
\hline 17 & 1 & 2 \\
\hline 20 & 2 & 1 \\
\hline 21 & 1 & $\mathbf{3 5}$ \\
\hline$X$ & 1 & \\
\hline Total & $\mathbf{4 2}$ & $\mathbf{8 3 . 7 3 \%}$ \\
\hline Accuracy & & \\
\hline & & \\
\hline & & 2 \\
\hline
\end{tabular}


Data for Patient: D

\begin{tabular}{|c|c|c|}
\hline Chromosome & $\begin{array}{c}\text { Observed } \\
\text { Mutations }\end{array}$ & $\begin{array}{c}\text { Predicted } \\
\text { Mutations }\end{array}$ \\
\hline 2 & 1 & 1 \\
\hline 3 & 1 & 1 \\
\hline 4 & 1 & 1 \\
\hline 5 & 1 & 1 \\
\hline 6 & 1 & 1 \\
\hline 8 & 2 & 1 \\
\hline 10 & 3 & 1 \\
\hline 16 & 2 & 2 \\
\hline 17 & 1 & 1 \\
\hline 18 & 1 & 1 \\
\hline$X$ & 1 & $\mathbf{1 2}$ \\
\hline Total & $\mathbf{1 5}$ & \\
\hline Accuracy & \multicolumn{2}{|c|}{} \\
\hline
\end{tabular}


Data for Patient: $E$

\begin{tabular}{|c|c|c|}
\hline Chromosome & $\begin{array}{l}\text { Observed } \\
\text { Mutations }\end{array}$ & $\begin{array}{c}\text { Predicted } \\
\text { Mutations }\end{array}$ \\
\hline 1 & 9 & 7 \\
\hline 2 & 15 & 11 \\
\hline 3 & 2 & 2 \\
\hline 4 & 12 & 9 \\
\hline 5 & 7 & 5 \\
\hline 6 & 7 & 5 \\
\hline 7 & 5 & 4 \\
\hline 8 & 3 & 2 \\
\hline 9 & 3 & 2 \\
\hline 10 & 4 & 3 \\
\hline 11 & 3 & 2 \\
\hline 12 & 3 & 2 \\
\hline 13 & 3 & 2 \\
\hline 14 & 2 & 2 \\
\hline 15 & 1 & 1 \\
\hline 16 & 2 & 1 \\
\hline 17 & 4 & 3 \\
\hline 18 & 4 & 3 \\
\hline 20 & 2 & 1 \\
\hline 21 & 2 & 2 \\
\hline 22 & 1 & 1 \\
\hline$X$ & 5 & 4 \\
\hline Total & 99 & 74 \\
\hline Accuracy & \multicolumn{2}{|c|}{$74.75 \%$} \\
\hline
\end{tabular}


Data for Patient: $F$

\begin{tabular}{|c|c|c|}
\hline Chromosome & $\begin{array}{c}\text { Observed } \\
\text { Mutations }\end{array}$ & $\begin{array}{c}\text { Predicted } \\
\text { Mutations }\end{array}$ \\
\hline 1 & 5 & 4 \\
\hline 2 & 14 & 11 \\
\hline 3 & 6 & 5 \\
\hline 4 & 6 & 4 \\
\hline 5 & 3 & 2 \\
\hline 6 & 10 & 8 \\
\hline 7 & 5 & 4 \\
\hline 8 & 4 & 3 \\
\hline 9 & 6 & 5 \\
\hline 10 & 3 & 2 \\
\hline 11 & 5 & 4 \\
\hline 12 & 4 & 3 \\
\hline 13 & 4 & 3 \\
\hline 14 & 4 & 3 \\
\hline 15 & 2 & 2 \\
\hline 17 & 3 & 2 \\
\hline 18 & 5 & 4 \\
\hline 19 & 2 & 2 \\
\hline 20 & 2 & 1 \\
\hline 21 & 1 & 1 \\
\hline 22 & 1 & 1 \\
\hline$X$ & 4 & 3 \\
\hline $\mathrm{Y}$ & 1 & 1 \\
\hline Total & 100 & 78 \\
\hline Accuracy & \multicolumn{2}{|c|}{$78.00 \%$} \\
\hline
\end{tabular}


Data for Patient: $G$

\begin{tabular}{|c|c|c|}
\hline Chromosome & $\begin{array}{c}\text { Observed } \\
\text { Mutations }\end{array}$ & $\begin{array}{c}\text { Predicted } \\
\text { Mutations }\end{array}$ \\
\hline 1 & 1 & 1 \\
\hline 2 & 1 & 1 \\
\hline 4 & 1 & 1 \\
\hline 7 & 1 & 1 \\
\hline 9 & 1 & 1 \\
\hline 12 & 1 & 0 \\
\hline 13 & 1 & 1 \\
\hline 14 & 1 & 1 \\
\hline 16 & 1 & 0 \\
\hline 18 & 1 & 1 \\
\hline 19 & 1 & 1 \\
\hline 21 & 1 & $\mathbf{1 0}$ \\
\hline Total & $\mathbf{1 2}$ & $\mathbf{8 3 . 3 3 \%}$ \\
\hline Accuracy & \multicolumn{2}{|l}{} \\
\hline
\end{tabular}


Data for Patient: $H$

\begin{tabular}{|c|c|c|}
\hline Chromosome & $\begin{array}{l}\text { Observed } \\
\text { Mutations }\end{array}$ & $\begin{array}{c}\text { Predicted } \\
\text { Mutations }\end{array}$ \\
\hline 1 & 5 & 4 \\
\hline 2 & 7 & 5 \\
\hline 3 & 3 & 2 \\
\hline 4 & 5 & 4 \\
\hline 5 & 5 & 4 \\
\hline 6 & 6 & 4 \\
\hline 7 & 1 & 1 \\
\hline 8 & 3 & 2 \\
\hline 9 & 3 & 2 \\
\hline 10 & 3 & 2 \\
\hline 11 & 3 & 2 \\
\hline 12 & 5 & 4 \\
\hline 14 & 2 & 2 \\
\hline 15 & 1 & 1 \\
\hline 16 & 2 & 2 \\
\hline 17 & 1 & 1 \\
\hline 18 & 1 & 1 \\
\hline 20 & 1 & 1 \\
\hline 22 & 1 & 1 \\
\hline$X$ & 7 & 6 \\
\hline Total & 65 & 51 \\
\hline Accuracy & \multicolumn{2}{|c|}{$78.46 \%$} \\
\hline
\end{tabular}




\section{Prediction results with summarized data including actual and predicted locations}

Below we have the results for our prediction indicating the actual location of the mutation and the predicted one. Similarly, we show the actual gene involved and the predicted gene involved. We also have a column called "difference" to indicate the error of our estimations. 


\section{PATIENT A}

$\begin{array}{llllll}\text { Chr } & \text { RelapseLocation } & \text { PredictedLocation } & \text { Difference } & \text { RelapseGene } & \text { PredictedGene } \\ 1 & 105905196 & 105903822 & & & \text { INTERGENIC-REGION } \\ 2 & 103478832 & 103478707 & -1374 & \text { INTERGENIC-REGION } & \text { LOC728815 } \\ 5 & 117968777 & 117966963 & -125 & \text { LOC728815 } & \text { INTERGENIC-REGION } \\ 6 & 117330941 & 117333649 & -1814 & \text { INTERGENIC-REGION } & \text { RFXDC1 } \\ 6 & 148467197 & 148465214 & 2708 & \text { RFXDC1 } & \text { INTERGENIC-REGION } \\ 7 & 120243306 & 120241310 & -1983 & \text { INTERGENIC-REGION } & \text { TSPAN12 } \\ 7 & 141400429 & 141399010 & -1996 & \text { TSPAN12 } & \text { MGAM } \\ 8 & 6610082 & 6611300 & -1419 & \text { MGAM } & \text { INTERGENIC-REGION } \\ 10 & 36300363 & 36297857 & 1218 & \text { INTERGENIC-REGION } & \text { INTERGENIC-REGION } \\ 12 & 36181843 & 36180237 & -2506 & \text { INTERGENIC-REGION } & \text { INTERGENIC-REGION } \\ 13 & 77038729 & 77037998 & -1606 & \text { INTERGENIC-REGION } & \text { SCEL } \\ 15 & 35693981 & 35696254 & -731 & \text { SCEL } & \text { INTERGENIC-REGION } \\ 15 & 96495601 & 96496583 & 2273 & \text { INTERGENIC-REGION } & \text { INTERGENIC-REGION } \\ 18 & 67016988 & 67019842 & 982 & \text { INTERGENIC-REGION } & \text { INTERGENIC-REGION } \\ 19 & 15919303 & 15919555 & 2854 & \text { INTERGENIC-REGION } & \text { INTERGENIC-REGION } \\ 22 & 46504211 & 46505232 & 252 & \text { INTERGENIC-REGION } & \text { INTERGENIC-REGION } \\ X & 7449501 & 7449885 & 1021 & \text { INTERGENIC-REGION } & \text { INTERGENIC-REGION } \\ X & 89181903 & 89179381 & 384 & \text { INTERGENIC-REGION } & \text { INTERGENIC-REGION }\end{array}$




\section{PATIENT B}

\begin{tabular}{|c|c|c|c|c|c|}
\hline Chr & RelapseLocation & PredictedLocation & Difference & RelapseGene & PredictedGene \\
\hline 1 & 18730737 & 18731528 & 791 & INTERGENIC-REGION & INTERGENIC-REGION \\
\hline 1 & 22831628 & 22830967 & -661 & INTERGENIC-REGION & INTERGENIC-REGION \\
\hline 1 & 89433568 & 89435914 & 2346 & GBP4 & GBP4 \\
\hline 1 & 119892131 & 119890585 & -1546 & INTERGENIC-REGION & INTERGENIC-REGION \\
\hline 1 & 164527242 & 164529039 & 1797 & INTERGENIC-REGION & INTERGENIC-REGION \\
\hline 2 & 4951026 & 4948442 & -2584 & INTERGENIC-REGION & INTERGENIC-REGION \\
\hline 2 & 18760057 & 18760752 & 695 & INTERGENIC-REGION & INTERGENIC-REGION \\
\hline 2 & 41983085 & 41981902 & -1183 & INTERGENIC-REGION & INTERGENIC-REGION \\
\hline 2 & 52337923 & 52337545 & -378 & INTERGENIC-REGION & INTERGENIC-REGION \\
\hline 2 & 53366757 & 53368759 & 2002 & INTERGENIC-REGION & INTERGENIC-REGION \\
\hline 2 & 114769762 & 114770766 & 1004 & INTERGENIC-REGION & INTERGENIC-REGION \\
\hline 2 & 137663285 & 137664050 & 765 & THSD7B & THSD7B \\
\hline 3 & 97423737 & 97422285 & -1452 & INTERGENIC-REGION & INTERGENIC-REGION \\
\hline 3 & 148730059 & 148727347 & -2712 & INTERGENIC-REGION & INTERGENIC-REGION \\
\hline 3 & 174114325 & 174114486 & 161 & SPATA16 & SPATA16 \\
\hline 4 & 17783989 & 17783525 & -464 & INTERGENIC-REGION & INTERGENIC-REGION \\
\hline 4 & 18912208 & 18913676 & 1468 & INTERGENIC-REGION & INTERGENIC-REGION \\
\hline 4 & 22759171 & 22761274 & 2103 & LOC643751 & LOC643751 \\
\hline 4 & 31779477 & 31778159 & -1318 & INTERGENIC-REGION & INTERGENIC-REGION \\
\hline
\end{tabular}




\begin{tabular}{ll}
4 & 103369618 \\
4 & 132134033 \\
5 & 27542961 \\
5 & 73771482 \\
5 & 95272060 \\
5 & 154221315 \\
6 & 11556067 \\
6 & 55539300 \\
6 & 89788729 \\
6 & 94679810 \\
6 & 98935123 \\
7 & 13405534 \\
7 & 25018068 \\
7 & 101609893 \\
7 & 101701575 \\
7 & 116559358 \\
8 & 73514564 \\
8 & 76476615 \\
9 & 7987216 \\
9 & 11413168 \\
10 & 21774153 \\
10 & 24053538 \\
10 & 37158144 \\
10 & 52992328 \\
10 & 100679446 \\
& \\
\hline
\end{tabular}

\begin{tabular}{l}
103367568 \\
132136548 \\
27545241 \\
73772512 \\
95274071 \\
154224153 \\
11553580 \\
55536902 \\
89786090 \\
94677079 \\
98933684 \\
13402864 \\
25018936 \\
101612118 \\
101703838 \\
116559716 \\
73511684 \\
76478306 \\
7987623 \\
11415994 \\
21775175 \\
24052997 \\
37159499 \\
52992927 \\
100679728 \\
\hline
\end{tabular}

-2050
2515
2280
1030
2011
2838
-2487
-2398
-2639
-2731
-1439
-2670
868
2225
2263
358
-2880
1691
407
2826
1022
-541
1355
599
282

INTERGENIC-REGION

INTERGENIC-REGION

INTERGENIC-REGION

INTERGENIC-REGION

ELL2

CNOT8

INTERGENIC-REGION

HMGCLL1

INTERGENIC-REGION

INTERGENIC-REGION

INTERGENIC-REGION

INTERGENIC-REGION

INTERGENIC-REGION

CUX1

CUX1

ST7

INTERGENIC-REGION

INTERGENIC-REGION

INTERGENIC-REGION

INTERGENIC-REGION

INTERGENIC-REGION

KIAA1217

INTERGENIC-REGION

PRKG1

HPSE2
INTERGENIC-REGION

INTERGENIC-REGION

INTERGENIC-REGION

INTERGENIC-REGION

ELL2

CNOT8

INTERGENIC-REGION

HMGCLL1

INTERGENIC-REGION

INTERGENIC-REGION

INTERGENIC-REGION

INTERGENIC-REGION

INTERGENIC-REGION

CUX1

CUX1

ST7

INTERGENIC-REGION

INTERGENIC-REGION

INTERGENIC-REGION

INTERGENIC-REGION

INTERGENIC-REGION

KIAA1217

INTERGENIC-REGION

PRKG1

HPSE2 


\begin{tabular}{|c|c|c|c|c|c|}
\hline 11 & 26915201 & 26913996 & -1205 & INTERGENIC-REGION & INTERGENIC-REGION \\
\hline 11 & 27181250 & 27184040 & 2790 & INTERGENIC-REGION & INTERGENIC-REGION \\
\hline 11 & 29982645 & 29982170 & -475 & INTERGENIC-REGION & INTERGENIC-REGION \\
\hline 12 & 61776966 & 61778798 & 1832 & INTERGENIC-REGION & INTERGENIC-REGION \\
\hline 12 & 72620513 & 72623219 & 2706 & INTERGENIC-REGION & INTERGENIC-REGION \\
\hline 12 & 82288736 & 82290376 & 1640 & INTERGENIC-REGION & INTERGENIC-REGION \\
\hline 13 & 35326695 & 35328951 & 2256 & DCLK1 & DCLK1 \\
\hline 13 & 53087241 & 53089305 & 2064 & INTERGENIC-REGION & INTERGENIC-REGION \\
\hline 13 & 56856089 & 56856249 & 160 & INTERGENIC-REGION & INTERGENIC-REGION \\
\hline 14 & 28018404 & 28019485 & 1081 & INTERGENIC-REGION & INTERGENIC-REGION \\
\hline 14 & 69464698 & 69464658 & -40 & SMOC1 & SMOC1 \\
\hline 15 & 39166414 & 39169210 & 2796 & INOC1 & INOC1 \\
\hline 15 & 88432939 & 88433205 & 266 & IDH2 & IDH2 \\
\hline 16 & 26547638 & 26546235 & -1403 & INTERGENIC-REGION & INTERGENIC-REGION \\
\hline 16 & 30758901 & 30759255 & 354 & \multicolumn{2}{|c|}{ LOC100129191 } \\
\hline 16 & 70613693 & 70614741 & 1048 & DHODH & DHODH \\
\hline 16 & 76093122 & 76094288 & 1166 & INTERGENIC-REGION & INTERGENIC-REGION \\
\hline 16 & 80961258 & 80960904 & -354 & INTERGENIC-REGION & INTERGENIC-REGION \\
\hline 17 & 7334949 & 7337664 & 2715 & POLR2A & POLR2A \\
\hline 17 & 10874727 & 10875744 & 1017 & INTERGENIC-REGION & INTERGENIC-REGION \\
\hline 17 & 27701282 & 27698390 & -2892 & MIRN632 & INTERGENIC-REGION \\
\hline 17 & 34007774 & 34005119 & -2655 & SNIP & SNIP \\
\hline 18 & 23266563 & 23267169 & 606 & FLJ45994 & FLJ45994 \\
\hline 18 & 36665237 & 36665827 & 590 & INTERGENIC-REGION & INTERGENIC-REGION \\
\hline 19 & 32989705 & 32990040 & 335 & LOC642290 & LOC642290 \\
\hline
\end{tabular}




$\begin{array}{llllll}19 & 41696897 & 41697985 & 1088 & \text { ZNF260 } \\ 20 & 17900146 & 17902446 & 2300 & \text { C20orf72 } & \text { ZNF260 } \\ 20 & 49730834 & 49729040 & -1794 & \text { ATP9A } 20 \text { orf72 } \\ 21 & 26930860 & 26928939 & -1921 & \text { INTERGENIC-REGION } & \text { ATP9A } \\ 21 & 27215461 & 27214843 & -618 & \text { ADTERGENIC-REGION } \\ 22 & 18266604 & 18269168 & 2564 & \text { TXNRD2 } & \text { ADAMTS5 } \\ \mathrm{X} & 24850996 & 24849917 & -1079 & \text { POLA1 } & \text { TXNRD2 } \\ \mathrm{X} & 33727134 & 33727059 & -75 & \text { INTERGENIC-REGION } & \text { INTELA1 } \\ \mathrm{Y} & 8487910 & 8485327 & -2583 & \text { INTERGENIC-REGION } & \text { INTERGENIC-REGION } \\ \mathrm{Y} & 16302321 & 16300318 & -2003 & \text { INTERGENIC-REGION } & \text { INTERGENIC-REGION }\end{array}$




\section{PATIENT C}

\begin{tabular}{|c|c|c|c|c|c|}
\hline Chr & RelapseLocation & PredictedLocation & Difference & RelapseGene & PredictedGene \\
\hline 1 & 107357719 & 107358018 & 299 & INTERGENIC-REGION & INTERGENIC-REGION \\
\hline 1 & 179307551 & 179309065 & 1514 & INTERGENIC-REGION & INTERGENIC-REGION \\
\hline 1 & 218018901 & 218020628 & 1727 & INTERGENIC-REGION & INTERGENIC-REGION \\
\hline 2 & 184186967 & 184186891 & -76 & INTERGENIC-REGION & INTERGENIC-REGION \\
\hline 2 & 213886052 & 213884438 & -1614 & SPAG16 & SPAG16 \\
\hline 3 & 21310708 & 21307866 & -2842 & INTERGENIC-REGION & INTERGENIC-REGION \\
\hline 3 & 43135962 & 43138381 & 2419 & INTERGENIC-REGION & INTERGENIC-REGION \\
\hline 4 & 26362673 & 26362567 & -106 & TBC1D19 & TBC1D19 \\
\hline 5 & 22595465 & 22595335 & -130 & $\mathrm{CDH} 12$ & $\mathrm{CDH} 12$ \\
\hline 5 & 93857376 & 93859895 & 2519 & INTERGENIC-REGION & INTERGENIC-REGION \\
\hline 6 & 40467367 & 40464779 & -2588 & LRFN2 & INTERGENIC-REGION \\
\hline 6 & 95631430 & 95629374 & -2056 & INTERGENIC-REGION & INTERGENIC-REGION \\
\hline 7 & 85476859 & 85474442 & -2417 & INTERGENIC-REGION & INTERGENIC-REGION \\
\hline 7 & 120917535 & 120914752 & -2783 & LOC392979 & LOC392979 \\
\hline 8 & 25022615 & 25022103 & -512 & INTERGENIC-REGION & INTERGENIC-REGION \\
\hline 8 & 34167561 & 34165529 & -2032 & INTERGENIC-REGION & INTERGENIC-REGION \\
\hline 8 & 35476742 & 35478696 & 1954 & INTERGENIC-REGION & INTERGENIC-REGION \\
\hline 8 & 51256369 & 51253560 & -2809 & SNTG1 & SNTG1 \\
\hline
\end{tabular}




$\begin{array}{llllll}9 & 36887024 & 36889733 & 2709 & \text { PAX5 } & \text { PAX5 } \\ 9 & 137816463 & 137818783 & 2320 & \text { KCNT1 } & \text { KCNT1 } \\ 11 & 23583427 & 23584671 & 1244 & \text { INTERGENIC-REGION } & \text { INTERGENIC-REGION } \\ 11 & 40183906 & 40182763 & -1143 & \text { LRRC4C } & \text { LRRC4C } \\ 12 & 9702101 & 9703657 & 1556 & \text { LOC374443 } & \text { INTERGENIC-REGION } \\ 12 & 22283439 & 22281122 & -2317 & \text { ST8SIA1 } & \text { ST8SIA1 } \\ 13 & 60889651 & 60891444 & 1793 & \text { INTERGENIC-REGION } & \text { INTERGENIC-REGION } \\ 13 & 92466026 & 92467721 & 1695 & \text { INTERGENIC-REGION } & \text { INTERGENIC-REGION } \\ 14 & 47961450 & 47963585 & 2135 & \text { INTERGENIC-REGION } & \text { INTERGENIC-REGION } \\ 14 & 82608644 & 82607776 & -868 & \text { INTERGENIC-REGION } & \text { INTERGENIC-REGION } \\ 16 & 7941529 & 7943725 & 2196 & \text { INTERGENIC-REGION } & \text { INTERGENIC-REGION } \\ 16 & 58050635 & 58052689 & 2054 & \text { INTERGENIC-REGION } & \text { INTERGENIC-REGION } \\ 17 & 67861248 & 67859454 & -1794 & \text { INTERGENIC-REGION } & \text { INTERGENIC-REGION } \\ 20 & 17261178 & 17262629 & 1451 & \text { PCSK2 } & \text { PCSK2 } \\ 20 & 58861532 & 58863711 & 2179 & \text { INTERGENIC-REGION } & \text { INTERGENIC-REGION } \\ 21 & 23370033 & 23372348 & 2315 & \text { INTERGENIC-REGION } & \text { LOC100130310 } \\ X & 86570002 & 86570879 & 877 & \text { INTERGENIC-REGION } & \text { INTERGENIC-REGION }\end{array}$




\section{PATIENT D}

$\begin{array}{llllll}\text { Chr } & \text { RelapseLocation } & \text { PredictedLocation } & \text { Difference } & \text { RelapseGene } & \text { PredictedGene } \\ & & & & & \\ 2 & 1119223 & 1118090 & -1133 & \text { SNTG2 } & \text { SNTG2 } \\ 3 & 183065520 & 183064291 & -1229 & \text { INTERGENIC-REGION } & \text { INTERGENIC-REGION } \\ 4 & 185168709 & 185166707 & -2002 & \text { STOX2 } & \text { STOX2 } \\ 5 & 162177435 & 162174604 & -2831 & \text { INTERGENIC-REGION } & \text { INTERGENIC-REGION } \\ 6 & 93462438 & 93461387 & -1051 & \text { INTERGENIC-REGION } & \text { INTERGENIC-REGION } \\ 8 & 3293986 & 3295797 & 1811 & \text { CSMD1 } & \text { CSMD1 } \\ 10 & 84453341 & 84452336 & -1005 & \text { NRG3 } & \text { NRG3 } \\ 16 & 61747374 & 61747530 & 156 & \text { INTERGENIC-REGION } & \text { INTERGENIC-REGION } \\ 16 & 62989048 & 62988198 & -850 & \text { INTERGENIC-REGION } & \text { INTERGENIC-REGION } \\ 17 & 22045352 & 22042405 & -2947 & \text { INTERGENIC-REGION } & \text { INTERGENIC-REGION } \\ 18 & 42887822 & 42889970 & 2148 & \text { HDHD2 } & \text { HDHD2 } \\ X & 121434334 & 121431398 & -2936 & \text { INTERGENIC-REGION } & \text { INTERGENIC-REGION }\end{array}$




\section{PATIENT E}

\begin{tabular}{|c|c|c|c|c|c|}
\hline Chr & RelapseLocation & PredictedLocation & Difference & RelapseGene & PredictedGene \\
\hline 1 & 44944905 & 44945092 & 187 & MGC33556 & MGC33556 \\
\hline 1 & 56758799 & 56755847 & -2952 & PPAP2B & PРAP2B \\
\hline 1 & 62329867 & 62328205 & -1662 & INADL & INADL \\
\hline 1 & 80186452 & 80184770 & -1682 & INTERGENIC-REGION & INTERGENIC-REGION \\
\hline 1 & 162652104 & 162653254 & 1150 & INTERGENIC-REGION & INTERGENIC-REGION \\
\hline 1 & 193708003 & 193707136 & -867 & INTERGENIC-REGION & INTERGENIC-REGION \\
\hline 1 & 193987679 & 193985578 & -2101 & INTERGENIC-REGION & INTERGENIC-REGION \\
\hline 2 & 16261487 & 16259478 & -2009 & INTERGENIC-REGION & INTERGENIC-REGION \\
\hline 2 & 79424915 & 79425833 & 918 & INTERGENIC-REGION & INTERGENIC-REGION \\
\hline 2 & 81008756 & 81008969 & 213 & INTERGENIC-REGION & INTERGENIC-REGION \\
\hline 2 & 83365574 & 83367876 & 2302 & INTERGENIC-REGION & INTERGENIC-REGION \\
\hline 2 & 121062191 & 121062952 & 761 & INTERGENIC-REGION & INTERGENIC-REGION \\
\hline 2 & 121512897 & 121514104 & 1207 & INTERGENIC-REGION & INTERGENIC-REGION \\
\hline 2 & 125156383 & 125157880 & 1497 & CNTNAP5 & CNTNAP5 \\
\hline 2 & 125314902 & 125317068 & 2166 & CNTNAP5 & CNTNAP5 \\
\hline 2 & 132126629 & 132126964 & 335 & INTERGENIC-REGION & INTERGENIC-REGION \\
\hline 2 & 136723675 & 136726085 & 2410 & INTERGENIC-REGION & INTERGENIC-REGION \\
\hline 2 & 151445176 & 151443514 & -1662 & INTERGENIC-REGION & INTERGENIC-REGION \\
\hline
\end{tabular}




\begin{tabular}{ll}
3 & 1887762 \\
3 & 175001625 \\
4 & 20084861 \\
4 & 30772025 \\
4 & 31398117 \\
4 & 93687647 \\
4 & 96814327 \\
4 & 105371233 \\
4 & 111770497 \\
4 & 137387938 \\
4 & 137620857 \\
5 & 21248739 \\
5 & 22482248 \\
5 & 22495984 \\
5 & 22744459 \\
5 & 45004983 \\
6 & 20902367 \\
6 & 50040060 \\
6 & 66897241 \\
6 & 87126465 \\
6 & 94961835 \\
7 & 13598141 \\
7 & 47290289 \\
7 & 68773299 \\
7 & 147006720 \\
& \\
\hline
\end{tabular}

1887661
175001097
20083013
30773500
31399931
93687017
96813949
105370352
111769372
137387568
137618498
21250383
22483703
22494710
22743789
45004149
20899506
50040552
66900208
87126336
94961835
13595372
47289020
68774264
147009358

-101
-528
-1848
1475
1814
-630
-378
-881
-1125
-370
-2359
1644
1455
-1274
-670
-834
-2861
492
2967
-129
0
-2769
-1269
965
2638

INTERGENIC-REGION

NLGN1

SLIT2

INTERGENIC-REGION

INTERGENIC-REGION

GRID2

INTERGENIC-REGION

INTERGENIC-REGION

PITX2

INTERGENIC-REGION

INTERGENIC-REGION

INTERGENIC-REGION

CDH12

CDH12

CDH12

INTERGENIC-REGION

CDKAL1

INTERGENIC-REGION

INTERGENIC-REGION

INTERGENIC-REGION

INTERGENIC-REGION

INTERGENIC-REGION

TNS3

AUTS2

CNTNAP2
INTERGENIC-REGION

NLGN1

SLIT2

INTERGENIC-REGION

INTERGENIC-REGION

GRID2

INTERGENIC-REGION

INTERGENIC-REGION

PITX2

INTERGENIC-REGION

INTERGENIC-REGION

INTERGENIC-REGION

CDH12

CDH12

$\mathrm{CDH} 12$

INTERGENIC-REGION

CDKAL1

INTERGENIC-REGION

INTERGENIC-REGION

INTERGENIC-REGION

INTERGENIC-REGION

INTERGENIC-REGION

TNS3

AUTS2

CNTNAP2 


\begin{tabular}{|c|c|c|c|c|c|}
\hline 8 & 4788849 & 4787918 & -931 & CSMD1 & CSMD1 \\
\hline 8 & 23806889 & 23805931 & -958 & INTERGENIC-REGION & INTERGENIC-REGION \\
\hline 9 & 12110080 & 12111967 & 1887 & INTERGENIC-REGION & INTERGENIC-REGION \\
\hline 9 & 28701959 & 28704866 & 2907 & LINGO2 & LINGO2 \\
\hline 10 & 52502567 & 52503933 & 1366 & PRKG1 & PRKG1 \\
\hline 10 & 55997651 & 55997740 & 89 & PCDH15 & PCDH15 \\
\hline 10 & 56392820 & 56391742 & -1078 & INTERGENIC-REGION & INTERGENIC-REGION \\
\hline 11 & 61267618 & 61269091 & 1473 & DAGLA & DAGLA \\
\hline 11 & 64146939 & 64144929 & -2010 & NRXN2 & NRXN2 \\
\hline 12 & 83033586 & 83033124 & -462 & INTERGENIC-REGION & INTERGENIC-REGION \\
\hline 12 & 93956009 & 93955103 & -906 & $\mathrm{NR} 2 \mathrm{C} 1$ & $\mathrm{NR} 2 \mathrm{C} 1$ \\
\hline 13 & 57139096 & 57136968 & -2128 & PCDH17 & PCDH17 \\
\hline 13 & 62444093 & 62442127 & -1966 & INTERGENIC-REGION & INTERGENIC-REGION \\
\hline 14 & 46290472 & 46290215 & -257 & INTERGENIC-REGION & INTERGENIC-REGION \\
\hline 14 & 105609184 & 105611251 & 2067 & INTERGENIC-REGION & INTERGENIC-REGION \\
\hline 15 & 76418530 & 76417893 & -637 & INTERGENIC-REGION & INTERGENIC-REGION \\
\hline 16 & 26981824 & 26984046 & 2222 & INTERGENIC-REGION & INTERGENIC-REGION \\
\hline 17 & 3004064 & 3003465 & -599 & OR1P1P & INTERGENIC-REGION \\
\hline 17 & 13501009 & 13499899 & -1110 & INTERGENIC-REGION & INTERGENIC-REGION \\
\hline 17 & 31379333 & 31379326 & -7 & INTERGENIC-REGION & INTERGENIC-REGION \\
\hline 18 & 5846231 & 5846301 & 70 & INTERGENIC-REGION & INTERGENIC-REGION \\
\hline 18 & 29678931 & 29677804 & -1127 & INTERGENIC-REGION & INTERGENIC-REGION \\
\hline 18 & 37355263 & 37356895 & 1632 & INTERGENIC-REGION & INTERGENIC-REGION \\
\hline 20 & 12364137 & 12361276 & -2861 & INTERGENIC-REGION & INTERGENIC-REGION \\
\hline 21 & 21114306 & 21113277 & -1029 & INTERGENIC-REGION & INTERGENIC-REGION \\
\hline
\end{tabular}




$\begin{array}{ll}21 & 27426278 \\ 22 & 49192948 \\ X & 23373203 \\ X & 88250191 \\ X & 97368810 \\ X & 116745872\end{array}$

27427344
49194052
23375572
88250874
97370397
116748160

1066
1104
2369
683
1587
2288

INTERGENIC-REGION

SAPS2

INTERGENIC-REGION

INTERGENIC-REGION

INTERGENIC-REGION

INTERGENIC-REGION

\section{PATIENT F}

\section{Chr RelapseLocation}

92405867
92814472
96583536
111864509
18769914
22359545
50857536
66491986
83614631
84115889
126135788

\section{PredictedLocation}

$$
\begin{aligned}
& 92406858 \\
& 92813494 \\
& 96582384 \\
& 111865538 \\
& 18768780 \\
& 22361976 \\
& 50855234 \\
& 66494804 \\
& 83612098 \\
& 84116667 \\
& 126137404
\end{aligned}
$$

\section{Difference RelapseGene}

991
-978
-1152
1029
-1134
2431
-2302
2818
-2533
778
1616

EVI5

ADORA3

NRXN1
INTERGENIC-REGION SAPS2

INTERGENIC-REGION INTERGENIC-REGION INTERGENIC-REGION INTERGENIC-REGION

\section{PredictedGene}

INTERGENIC-REGION

LOC 100132258

INTERGENIC-REGION

INTERGENIC-REGION

INTERGENIC-REGION

INTERGENIC-REGION

INTERGENIC-REGION

INTERGENIC-REGION
INTERGENIC-REGION
EVI5
LOC100132258
ADORA3
INTERGENIC-REGION
INTERGENIC-REGION
NRXN1
INTERGENIC-REGION
INTERGENIC-REGION
INTERGENIC-REGION
INTERGENIC-REGION 


\begin{tabular}{ll}
2 & 167238182 \\
2 & 172352355 \\
2 & 173346781 \\
2 & 184179988 \\
3 & 43881260 \\
3 & 46659901 \\
3 & 96394238 \\
3 & 139135471 \\
3 & 180628899 \\
4 & 27863801 \\
4 & 65007608 \\
4 & 123096911 \\
4 & 132093722 \\
5 & 23368167 \\
5 & 56807873 \\
6 & 126353 \\
6 & 9844264 \\
6 & 68090304 \\
6 & 77430774 \\
6 & 86029763 \\
6 & 94768681 \\
6 & 109550234 \\
6 & 112245364 \\
7 & 13880370 \\
7 & 39559272 \\
& \\
\hline
\end{tabular}

167237566
172354666
173345732
184177573
43883533
46659510
96395455
139135228
180631315
27863829
65008077
123098079
132093932
23369740
56807941
129210
9845543
68090598
77430160
86029106
94769618
109548825
112244333
13882510
39559474

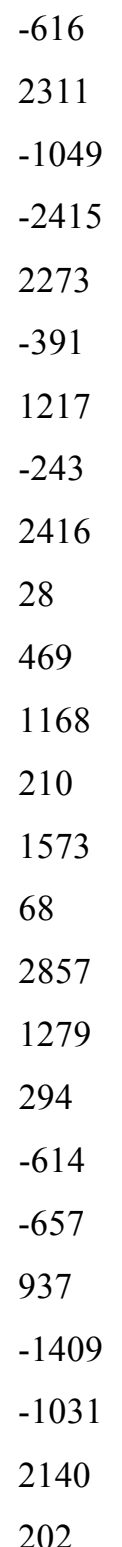

INTERGENIC-REGION

SLC25A12

RAPGEF4

INTERGENIC-REGION

INTERGENIC-REGION

INTERGENIC-REGION

INTERGENIC-REGION

INTERGENIC-REGION

GNB4

INTERGENIC-REGION

INTERGENIC-REGION

INTERGENIC-REGION

INTERGENIC-REGION

INTERGENIC-REGION

INTERGENIC-REGION

INTERGENIC-REGION

INTERGENIC-REGION

INTERGENIC-REGION

INTERGENIC-REGION

INTERGENIC-REGION

INTERGENIC-REGION

C6orf182

FYN

INTERGENIC-REGION

INTERGENIC-REGION
INTERGENIC-REGION

SLC25A12

RAPGEF4

INTERGENIC-REGION

INTERGENIC-REGION

INTERGENIC-REGION

INTERGENIC-REGION

INTERGENIC-REGION

GNB4

INTERGENIC-REGION

INTERGENIC-REGION

INTERGENIC-REGION

INTERGENIC-REGION

INTERGENIC-REGION

INTERGENIC-REGION

INTERGENIC-REGION

INTERGENIC-REGION

INTERGENIC-REGION

INTERGENIC-REGION

INTERGENIC-REGION

INTERGENIC-REGION

C6orf182

FYN

INTERGENIC-REGION

INTERGENIC-REGION 


$\begin{array}{ll}7 & 55323684 \\ 7 & 104147320 \\ 8 & 63596628 \\ 8 & 72857393 \\ 8 & 118381845 \\ 9 & 454456 \\ 9 & 80165699 \\ 9 & 110146992 \\ 9 & 118884413 \\ 9 & 126126705 \\ 10 & 2683387 \\ 10 & 6584079 \\ 11 & 10203546 \\ 11 & 40438832 \\ 11 & 55428174 \\ 11 & 87891301 \\ 12 & 38297190 \\ 12 & 44240246 \\ 12 & 79813281 \\ 13 & 55417148 \\ 13 & 70427767 \\ 13 & 81090538 \\ 14 & 31667574 \\ 14 & 33154434 \\ 14 & 56091064\end{array}$

\begin{tabular}{l}
55322359 \\
104147939 \\
63594602 \\
72854439 \\
118381895 \\
453609 \\
80166263 \\
110149359 \\
118884831 \\
126128087 \\
2682198 \\
6585226 \\
10200736 \\
40440545 \\
55430431 \\
87893310 \\
38294864 \\
44239417 \\
79812561 \\
55419652 \\
70426376 \\
81090305 \\
31668810 \\
33153004 \\
56089536 \\
\hline
\end{tabular}

-1325
619
-2026
-2954
50
-847
564
2367
418
1382
-1189
1147
-2810
1713
2257
2009
-2326
-829
-720
2504
-1391
-233
1236
-1430
-1528

INTERGENIC-REGION

LHFPL3

NKAIN3

INTERGENIC-REGION

INTERGENIC-REGION

DOCK8

INTERGENIC-REGION

INTERGENIC-REGION

ASTN2

NEK6

INTERGENIC-REGION

PRKCQ

SBF2

INTERGENIC-REGION

OR5W1P

GRM5

$\mathrm{ABCD} 2$

INTERGENIC-REGION

LIN7A

INTERGENIC-REGION

INTERGENIC-REGION

INTERGENIC-REGION

ARHGAP5

NPAS3

INTERGENIC-REGION
INTERGENIC-REGION

LHFPL3

NKAIN3

INTERGENIC-REGION

INTERGENIC-REGION

DOCK8

INTERGENIC-REGION

INTERGENIC-REGION

ASTN2

NEK6

INTERGENIC-REGION PRKCQ

SBF2

INTERGENIC-REGION

INTERGENIC-REGION

GRM5

$\mathrm{ABCD} 2$

INTERGENIC-REGION

LIN7A

INTERGENIC-REGION

INTERGENIC-REGION

INTERGENIC-REGION

ARHGAP5

NPAS3

INTERGENIC-REGION 


$\begin{array}{llllll}15 & 40851227 & 40850495 & -732 & \text { TTBK2 } & \text { TTBK2 } \\ 15 & 79991574 & 79992556 & 982 & \text { INTERGENIC-REGION } & \text { INTERGENIC-REGION } \\ 17 & 39843379 & 39841478 & -1901 & \text { GPATCH8 } & \text { GPATCH8 } \\ 17 & 45343100 & 45344560 & 1460 & \text { INTERGENIC-REGION } & \text { INTERGENIC-REGION } \\ 18 & 5053185 & 5051558 & -1627 & \text { INTERGENIC-REGION } & \text { INTERGENIC-REGION } \\ 18 & 5938070 & 5938026 & -44 & \text { INTERGENIC-REGION } & \text { INTERGENIC-REGION } \\ 18 & 11401121 & 11401673 & 552 & \text { INTERGENIC-REGION } & \text { INTERGENIC-REGION } \\ 18 & 39337589 & 39338647 & 1058 & \text { INTERGENIC-REGION } & \text { INTERGENIC-REGION } \\ 19 & 5893322 & 5895546 & 2224 & \text { RANBP3 } & \text { RANBP3 } \\ 19 & 43341487 & 43339217 & -2270 & \text { SIPA1L3 } & \text { SIPA1L3 } \\ 20 & 13210300 & 13213050 & 2750 & \text { C20orf82 } & \text { C20orf82 } \\ 21 & 42042422 & 42039541 & -2881 & \text { RIPK4 } & \text { RIPK4 } \\ 22 & 47687788 & 47685465 & -2323 & \text { INTERGENIC-REGION } & \text { INTERGENIC-REGION } \\ \mathrm{X} & 93658417 & 93660653 & 2236 & \text { INTERGENIC-REGION } & \text { INTERGENIC-REGION } \\ \mathrm{X} & 104960175 & 104962733 & 2558 & \text { NRK } & \text { NRK } \\ \mathrm{X} & 111343447 & 111345610 & 2163 & \text { ZCCHC16 } & \text { ZCCHC16 } \\ \mathrm{Y} & 7721445 & 7723250 & 1805 & \text { INTERGENIC-REGION } & \text { INTERGENIC-REGION }\end{array}$




\section{PATIENT G}

$\begin{array}{lll}\text { Chr } & \text { RelapseLocation } & \text { PredictedLo } \\ & & \\ & & \\ 1 & 79586157 & 79587862 \\ 2 & 147777631 & 147775238 \\ 4 & 142853353 & 142851908 \\ 7 & 19896920 & 19895976 \\ 9 & 25271855 & 25270748 \\ 13 & 91834507 & 91836278 \\ 14 & 25426007 & 25427524 \\ 18 & 6593666 & 6595162 \\ 19 & 1339089 & 1339688 \\ 21 & 30692169 & 30694843\end{array}$

Difference RelapseGene

1705

$-2393$

$-1445$

$-944$

$-1107$

1771

1517

1496

599

2674
INTERGENIC-REGION

INTERGENIC-REGION

IL15

INTERGENIC-REGION

INTERGENIC-REGION

GPC5

INTERGENIC-REGION

INTERGENIC-REGION

NDUFS7

INTERGENIC-REGION

\section{PredictedGene}

INTERGENIC-REGION

INTERGENIC-REGION

IL15

INTERGENIC-REGION

INTERGENIC-REGION

GPC5

INTERGENIC-REGION

INTERGENIC-REGION

NDUFS7

INTERGENIC-REGION 


\section{PATIENT H}

$\begin{array}{llllll}\text { Chr } & \text { RelapseLocation } & \text { PredictedLocation } & \text { Difference } & \text { RelapseGene } & \text { PredictedGene } \\ & & & & & \\ 1 & 28395117 & 28396128 & 1011 & \text { INTERGENIC-REGION } & \text { INTERGENIC-REGION } \\ 1 & 55348624 & 55345772 & -2852 & \text { USP24 } & \text { USP24 } \\ 1 & 80466160 & 80466553 & 393 & \text { INTERGENIC-REGION } & \text { INTERGENIC-REGION } \\ 1 & 104112500 & 104114609 & 2109 & \text { INTERGENIC-REGION } & \text { INTERGENIC-REGION } \\ 2 & 4358173 & 4355389 & -2784 & \text { INTERGENIC-REGION } & \text { INTERGENIC-REGION } \\ 2 & 14285436 & 14284784 & -652 & \text { INTERGENIC-REGION } & \text { INTERGENIC-REGION } \\ 2 & 106766704 & 106769370 & 2666 & \text { INTERGENIC-REGION } & \text { INTERGENIC-REGION } \\ 2 & 119250670 & 119251555 & 885 & \text { INTERGENIC-REGION } & \text { INTERGENIC-REGION } \\ 2 & 155081458 & 155083587 & 2129 & \text { INTERGENIC-REGION } & \text { INTERGENIC-REGION } \\ 3 & 1799169 & 1800181 & 1012 & \text { INTERGENIC-REGION } & \text { INTERGENIC-REGION } \\ 3 & 20956204 & 20956611 & 407 & \text { INTERGENIC-REGION } & \text { INTERGENIC-REGION } \\ 4 & 46955181 & 46952231 & -2950 & \text { GABRB1 } & \text { GABRB1 } \\ 4 & 149316450 & 149317894 & 1444 & \text { NR3C2 } & \text { NR3C2 } \\ 4 & 150025553 & 150024817 & -736 & \text { INTERGENIC-REGION } & \text { INTERGENIC-REGION } \\ 4 & 158256256 & 158253511 & -2745 & \text { GLRB } & \text { GLRB } \\ 5 & 15823239 & 15821488 & -1751 & \text { FBXL7 } & \text { FBXL7 } \\ 5 & 29515287 & 29512926 & -2361 & \text { INTERGENIC-REGION } & \text { INTERGENIC-REGION }\end{array}$




$\begin{array}{ll}5 & 104466311 \\ 5 & 113840798 \\ 6 & 9855937 \\ 6 & 38352606 \\ 6 & 96112105 \\ 6 & 98495373 \\ 7 & 141689176 \\ 8 & 2568547 \\ 8 & 31261001 \\ 9 & 75985332 \\ 9 & 82308637 \\ 10 & 22510615 \\ 10 & 23382721 \\ 11 & 26651719 \\ 11 & 82054955 \\ 12 & 3464622 \\ 12 & 11883491 \\ 12 & 43022207 \\ 12 & 72136658 \\ 14 & 20830373 \\ 14 & 43499979 \\ 15 & 76806819 \\ 16 & 59519213 \\ 16 & 84039293 \\ 17 & 22057902\end{array}$

104465825
113840471
9853948
38355567
96114311
98496129
141686364
2567553
31259469
75984229
82311378
22508285
23380837
26654173
82055363
3467549
11884936
43021782
72138814
20828361
43498964
76809320
59518116
84040691
22059434

-486
-327
-1989
2961
2206
756
-2812
-994
-1532
-1103
2741
-2330
-1884
2454
408
2927
1445
-425
2156
-2012
-1015
2501
-1097
1398
1532

INTERGENIC-REGION

KCNN2

INTERGENIC-REGION

BTBD9

INTERGENIC-REGION

INTERGENIC-REGION

TRB@

INTERGENIC-REGION

INTERGENIC-REGION

INTERGENIC-REGION

INTERGENIC-REGION

INTERGENIC-REGION

INTERGENIC-REGION

SLC5A12

INTERGENIC-REGION

INTERGENIC-REGION

ETV6

TMEM117

INTERGENIC-REGION

RPGRIP1

INTERGENIC-REGION

LOC646934

INTERGENIC-REGION

INTERGENIC-REGION

INTERGENIC-REGION
INTERGENIC-REGION

KCNN2

INTERGENIC-REGION

BTBD9

INTERGENIC-REGION

INTERGENIC-REGION

TRB@

INTERGENIC-REGION

INTERGENIC-REGION

INTERGENIC-REGION

INTERGENIC-REGION

INTERGENIC-REGION

INTERGENIC-REGION

SLC5A12

INTERGENIC-REGION

INTERGENIC-REGION

ETV6

TMEM117

INTERGENIC-REGION

RPGRIP1

INTERGENIC-REGION

LOC646934

INTERGENIC-REGION

INTERGENIC-REGION

INTERGENIC-REGION 


$\begin{array}{lll}18 & 13846987 & 13844333 \\ 20 & 43469052 & 43469585 \\ 22 & 24752889 & 24753260 \\ X & 6998334 & 6996308 \\ X & 104572042 & 104570775 \\ X & 114186799 & 114188858 \\ X & 121017574 & 121014781 \\ X & 127355500 & 127356514 \\ X & 127868848 & 127869973\end{array}$

-2654
533
371
-2026
-1267
2059
-2793
1014
1125

$-2654$

533

$-2026$

$-1267$

2059

$-2793$

1125
INTERGENIC-REGION

SYS1-DBNDD2

MYO18B

HDHD1A

IL1RAPL2

INTERGENIC-REGION

INTERGENIC-REGION

INTERGENIC-REGION

INTERGENIC-REGION
INTERGENIC-REGION SYS1-DBNDD2

MYO18B

HDHD1A

IL1RAPL2

INTERGENIC-REGION

INTERGENIC-REGION

INTERGENIC-REGION

INTERGENIC-REGION 


\section{BIBLIOGRAPHY}

[1] Patel LR, Nykter M, Chen K, Zhang W: Cancer genome sequencing: Understanding malignancy as a disease of the genome, its conformation, and its evolution. Cancer letters 2012.

[2] Campo E: Whole genome profiling and other high throughput technologies in lymphoid neoplasms--current contributions and future hopes. Modern pathology : an official journal of the United States and Canadian Academy of Pathology, Inc 2013, 26 Suppl 1:S97-s110.

[3] Mardis ER, Wilson RK. Cancer genome sequencing: a review. Hum Mol Genet. 2009 Oct 15;18(R2):R163-8. doi: 10.1093/hmg/ddp396.

[4] Welch JS, Link DC: Genomics of AML: clinical applications of next-generation sequencing. Hematology / the Education Program of the American Society of Hematology American Society of Hematology Education Program 2011, 2011:30-35.

[5] Kilpivaara O, Aaltonen LA: Diagnostic cancer genome sequencing and the contribution of germline variants. Science (New York, NY) 2013, 339(6127):1559-1562.

[6] Erho N, Crisan A, Vergara IA, Mitra AP, Ghadessi M, Buerki C, Bergstralh EJ, Kollmeyer T, Fink S, Haddad Z et al: Discovery and validation of a prostate cancer genomic classifier that predicts early metastasis following radical prostatectomy. PloS one 2013, 8(6):e66855.

[7] Stretch C, Khan S, Asgarian N, Eisner R, Vaisipour S, Damaraju S, Graham K, Bathe OF, Steed H, Greiner R et al: Effects of sample size on differential gene expression, rank order and prediction accuracy of a gene signature. PloS one 2013, 8(6):e65380.

[8] Bao ZS, Zhang CB, Wang HJ, Yan W, Liu YW, Li MY, Zhang W: Whole-genome mRNA expression profiling identifies functional and prognostic signatures in patients with mesenchymal glioblastoma multiforme. CNS neuroscience \& therapeutics 2013, 19(9):714-720.

[9] Sanz-Pamplona R, Berenguer A, Cordero D, Riccadonna S, Sole X, Crous-Bou M, Guino E, Sanjuan X, Biondo S, Soriano A et al: Clinical value of prognosis gene expression signatures in colorectal cancer: a systematic review. PloS one 2012, 7(11):e48877.

[10] Srivastava M, Khurana P, Sugadev R: Lung cancer signature biomarkers: tissue specific semantic similarity based clustering of digital differential display (DDD) data. BMC research notes 2012, 5:617. 
[11] Bedolla RG, Gong J, Prihoda TJ, Yeh IT, Thompson IM, Ghosh R, Kumar AP: Predictive value of $\mathrm{Sp} 1 / \mathrm{Sp} 3 / \mathrm{FLIP}$ signature for prostate cancer recurrence. PloS one 2012, 7(9):e44917.

[12] Mustacchi G, Sormani MP, Bruzzi P, Gennari A, Zanconati F, Bonifacio D, Monzoni A, Morandi L: Identification and validation of a new set of five genes for prediction of risk in early breast cancer. International journal of molecular sciences 2013, 14(5):9686-9702.

[13] Ding L, Ley TJ, Larson DE, Miller CA, Koboldt DC, Welch JS, Ritchey JK, Young MA, Lamprecht T, McLellan MD et al: Clonal evolution in relapsed acute myeloid leukaemia revealed by whole-genome sequencing. Nature 2012, 481(7382):506-510.

[14] Flicek P, Ahmed I, Amode MR, Barrell D, Beal K, Brent S, Carvalho-Silva D, Clapham P, Coates G, Fairley S, Fitzgerald S, Gil L, García-Girón C, Gordon L, Hourlier T, Hunt S, Juettemann T, Kähäri AK, Keenan S, Komorowska M, Kulesha E, Longden I, Maurel T, McLaren WM, Muffato M, Nag R, Overduin B, Pignatelli M, Pritchard B, Pritchard E, Riat HS, Ritchie GR, Ruffier M, Schuster M, Sheppard D, Sobral D, Taylor K, Thormann A, Trevanion S, White S, Wilder SP, Aken BL, Birney E, Cunningham F, Dunham I, Harrow J, Herrero J, Hubbard TJ, Johnson N, Kinsella R, Parker A, Spudich G, Yates A, Zadissa A, Searle SM. Nucleic Acids Res. 2013 Jan;41(Database issue):D4855. doi: 10.1093/nar/gks1236. Epub 2012 Nov 30. Ensembl 2013.

[15] Christian Del Fabbro. Repeated sequences in bioinformatics: assembly, annotation and alignments. PhD Dissertation. Universita Degli Studi Di Udine, 2009.

[16] J.D. Watson and F.H.C. Crick, A Structure for Deoxyribose Nucleic Acid. Nature $171,1953$.

[17] R. Langridge, W. E. Seeds, H. R. Wilson, C. W. Hooper, M. H. F. Wilkins, and L. D. Hamilton.Molecular Structure of Deoxypentose Nucleic Acids. Nature 171, 1953.

[18] B. Alberts, A. Johnson, J. Lewis, M. Raff, K. Roberts, and P. Walters. Molecular Biology of the Cell. New York and London: Garland Science, 4th edition, 2002.

[19] Grechko VV. Repeated DNA sequences as an engine of biological diversification. Molecular Biology (Mosk). 2011 Sep-Oct;45(5):765-92.

[20] Jurka J, Kapitonov VV, Kohany O, Jurka MV. Repetitive sequences in complex genomes: structure and evolution. Annual Review Genomics Human Genetics 2007;8:241-59.

[21] M.B. Gerstein, C. Bruce, J.S. Rozowsky, D. Zheng, J. Du, J.O. Korbel, O. Emanuelsson, Z.D. Zhang, S. Weissman, and M. Snyder. What is a gene, postENCODE? History and updated definition. Genome Res, 17(6):669\{681, Jun 2007. 
[22] Rister J, Desplan C. Deciphering the genome's regulatory code: the many languages of DNA. Bioessays. 2010 May;32(5):381-4. doi: 10.1002/bies.200900197.

[23] http://www.cancer.gov

[24] http://biologypop.com/dna-mutation-info/

[25] Alex van Belkum, Stewart Scherer, Loek van Alphen, and Henri Verbrugh, "ShortSequence DNA Repeats in Prokaryotic Genomes," Microbiology and Molecular Biology Reviews, 62(2), 1998.

[26] Stefan Kurtz, Jomuna V. Choudhuri, Enno Ohlebusch, Chris Schleiermacher, Jens Stoye, and Robert Giegerich, "REPuter: The Manifold Applications of Repeat Analysis on a Genomic Scale,” Nucleic Acids Research, 29(22) (2001) 4633-4642.

[27] Guillaume Achaz, Eric Coissac, Pierre Netter, and Eduardo P. C. Rocha, "Associations between Inverted Repeats and the Structural Evolution of Bacterial Genomes," Genetics, 164(4), 2003.

[28] E. Lander et al., "Initial Sequencing and Analysis of theHuman Genome," Nature, 409(6822), 2001.

[29] Eduardo P. C. Rocha, Antoine Danchin, and Alain Viari, "Analysis of Long Repeats in Bacterial Genomes Reveals Alternative Evolutionary Mechanisms in Bacillus subtilis and Other Competent Prokaryotes," Molecular Biology and Evolution, 16(9), 1999.

[30] Masataka Tsuge, Ryuji Hamamoto, Fabio Pittella Silva, Yozo Ohnishi, Kazuaki Chayama, Naoyuki Kamatani, Yoichi Furukawa, and Yusuke Nakamura, "A Variable Number of Tandem Repeats Polymorphism in an E2F-1 Binding Element in the 5 flanking region of SMYD3 is a Risk Factor for Human Cancers," Nature Genetics, 37, 2005.

[31] Subbaya Subramanian, Rakesh Mishra, and Lalji Singh, "Genome-Wide Analysis of Microsatellite Repeats in Humans: Their Abundance and Density in Specific Genomic Regions,"Genome Biology, 4(2), 2003.

[32] A. Blanes and SJ. Diaz-Cano. Complementary analysis of microsatellite tumor profile and mismatch repair defects in colorectal carcinomas. World $\mathrm{J}$. Gastroenterol. 2006.

[33] Lucy R. Yates and Peter J. Campbell. Evolution of the Cancer Genome. Nat Rev Genet. 2012. 
[34] http://www.nature.com/scitable/content/examples-of-genetic-diseases-caused-byexpanding-27926

[35] Kathleen H. Burns and Jef D. Boeke. Human Transposon Tectonics. Cell Volum 149, Issue 4, May 11, 2012.

[36] Fang Y, Yao Q, Chen Z, Xiang J, William FE, Gibbs RA, Chen C: Genetic and molecular alterations in pancreatic cancer: Implications for personalized medicine. Medical science monitor: international medical journal of experimental and clinical research 2013, 19:916-926.

[37] Avery OT, Macleod CM, McCarty M: Studies On The Chemical Nature Of The Substance Inducing Transformation Of Pneumococcal Types : Induction Of Transformation By A Desoxyribonucleic Acid Fraction Isolated From Pneumococcus Type III. The Journal of experimental medicine 1944, 79(2):137-158.

[38] Steinman RM, Moberg CL: A triple tribute to the experiment that transformed biology. The Journal of experimental medicine 1994, 179(2):379-384.

[39] Watson JD, Crick FH: Genetical implications of the structure of deoxyribonucleic acid. Nature 1953, 171(4361):964-967.

[40] Lander ES, Linton LM, Birren B, Nusbaum C, Zody MC, Baldwin J, Devon K, Dewar K, Doyle M, FitzHugh W et al: Initial sequencing and analysis of the human genome. Nature 2001, 409(6822):860-921.

[41] Venter JC, Adams MD, Myers EW, Li PW, Mural RJ, Sutton GG, Smith HO, Yandell M, Evans CA, Holt RA et al: The sequence of the human genome. Science (New York, NY) 2001, 291(5507):1304-1351.

[42] Finishing the euchromatic sequence of the human genome. Nature 2004, 431(7011):931-945.

[43] Osoegawa K, Mammoser AG, Wu C, Frengen E, Zeng C, Catanese JJ, de Jong PJ: A bacterial artificial chromosome library for sequencing the complete human genome. Genome research 2001, 11(3):483-496.

[44] Mardis ER, Wilson RK: Cancer genome sequencing: a review. Human molecular genetics 2009, 18(R2):R163-168.

[45] Korf BR: Integration of genomics into medical practice. Discovery medicine 2013, 16(89):241-248.

[46] Jeff Bizzaro and Kenneth Marx, "Poly: A Quantitative Analysis Tool for Simple Sequence Repeat (SSR) Tracts in DNA," Biomed Central Bioinformatics, 4(1), 2003. 
[47] AkitoTaneda, "Adplot: Detection and Visualization of Repetitive Patterns in Complete Genomes,” Bioinformatics, 20(5) (2004) 701-708.

[48] Jeff Reneker and Chi-Ren Shyu, "Refined Repetitive Sequence Searches Utilizing a Fast Hash Function and Cross Species Information Retrievals," Biomed Central Bioinformatics, 6(1), 2005.

[49] Alkes L. Price, Neil C. Jones, and Pavel A. Pevzner, "De novo Identification of Repeat Families in Large Genomes," Bioinformatics, 21(suppl_1), 2005.

[50] S. Bachellier, J.-M. Clément, and M. Hofnung, "Short Palindrome Repetitive DNA Elements in Enterobacteria: A Survey," Research in Microbiology, 150, 1999.

[51] Tetsuhiko Yoshida, Nobuaki Obata1, and Kenji Oosawa, "Color-Coding Reveals Tandem Repeats in the Escherichia coli Genome," Journal of Molecular Biology, 298, 2000.

[52] G. Benson, "Tandem Repeats Finder: A Program to Analyze DNA Sequences," Nucleic Acids Research, 27(2), 1999.

[53] Adalberto T. Castelo, Wellington Martins, and Guang R. Gao, "TROLL: Tandem Repeat Occurrence Locator," Bioinformatics, 18(4), 2002.

[54] Roman Kolpakov, Ghizlane Bana, and Gregory Kucherov, "Mreps: Efficient and Flexible Detection of Tandem Repeats in DNA," Nucleic Acids Research, 31(13), 2003.

[55] D. Sharma, B. Issac, G. P. S. Raghava, and R. Ramaswamy, "Spectral Repeat Finder (SRF): Identification of Repetitive Sequences using Fourier Transformation," Bioinformatics, 20(9), 2004

[56] Alex van Belkuma, Willem van Leeuwena, Stewart Schererb, and Henri Verbrugha, "Occurrence and Structure-Function Relationship of Pentameric Short Sequence Repeats in Microbial Genomes," Research in Microbiology, 150, 1999.

[57] Natalia Volfovsky, Brian Haas, and Steven Salzberg, "A Clustering Method for Repeat Analysis in DNA Sequences," Genome Biology, 2(8), 2001.

[58] Mohamed Ibrahim Abouelhoda, EnnoOhlebusch, and Stefan Kurtz, "Optimal Exact String Matching Based on Suffix Arrays," in Proceedings of the Ninth International Symposium on String Processing and Information Retrieval. Springer-Verlag 2002.

[59] William R. Pearson. Using the FASTA Program to Search Protein and DNA Sequence Databases. Computer Analysis of Sequence Data. Methods in Molecular Biology Volume 25, 1994. 
[60] Stephen F. Altschul, Warren Gish, Webb Miller, Eugene W. Myers, David J. Lipman. Basic local alignment search tool. Journal of Molecular Biology, Volume 215, Issue 3, 1990.

[61] http://blast.ncbi.nlm.nih.gov/

[62] NCBI National Center for Biotechnology Information http://www.ncbi.nlm.nih.gov/

[63] Maja Tarailo-Graovac, Nansheng Chen. Using RepeatMasker to Identify Repetitive Elements in Genomic Sequences. Current Protocols in Bioinformatics, 2009.

[64] http://www.repeatmasker.org/

[65] http://nebc.nox.ac.uk/bioinformatics/docs/cross_match.html

[66] http://blast.advbiocomp.com/

[67] http://www.ebi.ac.uk/Tools/sss/wublast/

[68] http://www.repeatmasker.org/RMBlast.html

[69] http://cmgm.stanford.edu/ decypher/dna.html

[70] Bedell Joseph A., Korf Ian and Gish Warren. MaskerAid: A performance enhancement to RepeatMasker.. Bioinformatics 2000.

[71] Benson G. Tandem Repeats Finder: A program to analyze DNA sequences. Oxford University Press, Nucleic Acids Research 1999.

[72] Kurtz S and Chris S. REPuter: fast computation of maximal repeats in complete genomes.. Bioinformatics 1999.

[73] Stefan Kurtz . The Vmatch large scale sequence analysis software. www.vmatch.de, 2007.

[74] Mohamed Ibrahim Abouelhoda, Stefan Kurtz, and EnnoOhlebusch, "The Enhanced Suffix Array and Its Applications to Genome Analysis," in WABI '02: Proceedings of the Second International Workshop on Algorithms in Bioinformatics (Springer-Verlag, London, UK, 2002.

[75] UdiManber andGeneMyers, "Suffix Arrays: ANewMethod forOn-Line String Searches," SIAM Journal on Computing, 22(5), 1993. 
[76] Linhart C, Halperin Y, Shamir R. Transcription factor and microRNA motif discovery: the Amadeus platform and a compendium of metazoan target sets. Genome Res., 2008.

[77] http://acgt.cs.tau.ac.il/allegro/download.html

[78] Philip Machanick and Timothy L. Bailey. MEME-ChIP: motif analysis of large DNA datasets. Oxford Journals: Bioinformatics 2011.

[79] M. R. Stratton, P. J. Campbell, and P. A. Futreal, "The cancer genome," Nature, vol. 458, no. 7239, pp. 719-724, 2009.

[80] E. P. Reddy, R. K. Reynolds, E. Santos, and M. Barbacid, "A point mutation is responsible for the acquisition of trans- forming properties by the t 24 human bladder carcinoma oncogene," Nature, vol. 300, no. 5888, pp. 149-152, July 1981.

[81] C. Greenman, P. Stephens, and R. Smith, "Patterns of somatic mutation in human cancer genomes," Nature, vol. 446, no. 1, pp. 153-158, 2007.

[82] D. A. Benson, I. Karsch-Mizrachi, K. Clark, D. J. Lipman, J. Ostell, and E. W. Sayers, "Genbank," Nucleic acid research, vol. 39, pp. D32-D37, 2011.

[83] R. R. Voss, "Evolution of long-range fractal correlations and $1 / \mathrm{f}$ noise in dna base sequences," Physical Review Letters, vol. 68, no. 25, pp. 3805-3808, 1992.

[84] L. Prasad and S.S. Iyengar, "Wavelet Analysis with an Application to Image Processing",Chapman and Hall/CRC Press, June 1997, pp. 279.

[85] M. Sifuzzaman, M. R. Islam, and M. Z. Ali, "Application of wavelet transform and its advantages compared to fourier transform," Journal of Physical Sciences, vol. 13, pp. 121-134, 2009.

[86] C. Gargour, M. Gabrea, V. Ramachandran, and J. M. Lina, "A short introduction to wavelets and their applications," IEEE Circuits and Systems Magazine, vol. 9, pp. 5768, II Quarter 2009.

[87] D. Gabor, "Theory of communication," IEEE Radio Communi cation Engineering Journal, vol. 93, no. 26, pp. 429-457, 1946.

[88] A. Haar, "Zur theorie der orthogonalen funktionensysteme," Mathematische Annalen, vol. 69, no. 3, pp. 331-371, 1910.

[89] J. B. Allen and L. R. Rabiner, "A unified approach to short- time fourier analysis and synthesis," Proc. IEEE, vol. 65, no. 11, pp. 1558-1564, 1977. 
[90] S. G. Mallat, "A theory for multiresolution signal decomposition: the wavelet representation," Pattern Analysis andMachine Intelligence, IEEE Transactions on, vol. 11, no. 7, pp. 674-693, 1989.

[91] I. Daubechies, Ten Lectures on Wavelets, ser. CBMS-NSF Regional Conference Series in Applied Mathematics. Society for Industrial and Applied Mathematics, 1992.

[92] Tao Meng, Ahmed T. Soliman, Mei-Ling Shyu, Yimin Yang, Shu-Ching Chen, S. S. Iyengar, John Yordy, and Puneeth Iyengar, "Wavelet Analysis in Current Cancer Genome Research: A Survey," accepted for publication, IEEE/ACM Transactions on Computational Biology and Bioinformatics.

[93] R. Dulbecco, "A turning point in cancer research: sequencing the human genome," Science, vol. 231, no. 4742, pp. 1055-1056, 1986.

[94] L. Chin, W. C. Hahn, and G. Getz, "Making sense of cancer genomic data," Genes and Development, vol. 25, no. 6, pp. 534-555, 2011.

[95] E. V. Ball, P. D. Stenson, S. S. Abeysinghe, M. Krawczak, D. N. Cooper, and N. A. Chuzhanova, "Microdeletions and microinsertions causing human genetic disease: common mech anisms of mutagenesis and the role of local dna sequence complexity," Human mutation, vol. 26, no. 3, pp. 205-213, 2005.

[96] E. M. Kvikstad, F. Chiaromonte, and K. D. Makova, "Ride the wavelet: A multiscale analysis of genomic contexts flanking small insertions and deletions," Genome research, vol. 19 , no. 7, pp. 1153-1164, 2009

[97] P. L. Foster, A. J. Hanson, H. Lee, E. M. Popodi, and H. Tang, "On the mutational topology of the bacterial genome," G3: Genes, Genomes, Genetics, vol. 3, no. 3, pp. 399-407, 2013.

[98] L. Song. Computational analysis of genome-wide dna copy number changes. Ph.D. dissertation, Virginia Polytechnic Institute and State University, 2011.

[99] S. Ivakhno, T. Royce, A. J. Cox, D. J. Evers, R. K. Cheetham,and S. Tavaree, "Cnaseg - a novel framework for identification of copy number changes in cancer from second-generation sequencing data," Bioinformatics, vol. 26, no. 24, pp. 3051-3058, 2010.

[100] L. M. Tran, B. Zhang, Z. Zhang, C. Zhang, T. Xie, J. R. Lamb, H. Dai, E. E. Schadt, and J. Zhu, "Inferring causal genomic alterations in breast cancer using gene expression data," BMC Systems Biology, vol. 5, no. 121, 2011.

[101] E. Ben-Yaacov and Y. C. Eldar, "A fast and flexible method for the segmentation of acgh data," Bioinformatics, vol. 24, no. 16, pp. i139-i145, 2008. 
[102] K. C. Amarasinghe, J. Li, and S. K. Halgamuge, "Convex: copy number variation estimation in exome sequencing data using hmm," BMC bioinformatics, vol. 14, no. Suppl 2, p. S2,2013.

[103] S. Ivakhno, T. Royce, A. J. Cox, D. J. Evers, R. K. Cheetham, and S. Tavarẽ , "Cnaseg - a novel framework for identification of copy number changes in cancer from second generation sequencing data," Bioinformatics, vol. 26, no. 24, pp. 3051-3058, 2010 .

[104] A. M. Sarhan, "Wavelet-based feature extraction for dna microarray classification," Artificial Intelligence Review, vol. 39, no. 3, pp. 237-249, 2013.

[105] Y. Liu, U. Aickelin, J. Feyereisl, and L. G. Durrant, "Wavelet feature extraction and genetic algorithm for biomarker detection in colorectal cancer data," KnowledgeBased Systems, vol. 37, pp. S02-514, 2013.

[106] Timmermann B, Kerick M, Roehr C, Fischer A, Isau M, Boerno ST, Wunderlich A, Barmeyer C, Seemann P, Koenig J et al: Somatic mutation profiles of MSI and MSS colorectal cancer identified by whole exome next generation sequencing and bioinformatics analysis. PloS one 2010, 5(12):e15661.

[107] Roth A, Ding J, Morin R, Crisan A, Ha G, Giuliany R, Bashashati A, Hirst M, Turashvili G, Oloumi A et al: JointSNVMix: a probabilistic model for accurate detection of somatic mutations in normal/tumour paired next-generation sequencing data. Bioinformatics (Oxford, England) 2012, 28(7):907-913.

[108] http://www.cs.waikato.ac.nz/ml/weka/

[109] http://weka.wikispaces.com/Primer

[110] http://weka.sourceforge.net/doc/weka/core

[111] G. E. P. Box, W. G. Hunter and J. S. Hunter. Statistics for Experimenters. New York: Wilet, 1978, ch. 16.

[112] J. R. Kittrell, R. Mezaki and C. C. Watson. Estimation of parameteres for nonlinear least square analysis. Ind. Eng. Chem. vol. 57, no. 12, p 19, 1965.

[113] R. L. Plackett. Principles of Regression Analysis. New York: Oxford, 1960.

[114] P. M. Reilly. Statistical techniques for model-building. PhD dissertation, Univ. Wisconsin, Madison, 1966. 
[115] Iyengar and Rao, 1983 S.S. Iyengar, M.S. Rao. Statistical techniques in modelling of complex systems: single and multi-response models. IEEE Trans. Syst. Man Cybernet., SMC-13 (1983), pp. 175-189.

[116] http://www.stat.yale.edu/Courses/1997-98/101/linreg.htm

[117] http://www.stjude.org/downing-iom

[118] Frank E, Hall M, Trigg L, Holmes G, Witten IH: Data mining in bioinformatics using Weka. Bioinformatics (Oxford, England) 2004, 20(15):2479-2481.

[119] Chang, Chih-Chung; Lin, Chih-Jen (2011). "LIBSVM: A library for support vector machines". ACM Transactions on Intelligent Systems and Technology 2 (3).

[120]. Rasmussen, C. E. (2004). "Gaussian Processes in Machine Learning". Advanced Lectures on Machine Learning. Lecture Notes in Computer Science 3176. pp. 63-71

[121]. Lukaszyk, S. (2004) A new concept of probability metric and its applications in approximation of scattered data sets. Computational Mechanics, 33, 299-3004

[122] Ng SB, Turner EH, Robertson PD, Flygare SD, Bigham AW, Lee C, Shaffer T, Wong M, Bhattacharjee A, Eichler EE et al: Targeted capture and massively parallel sequencing of 12 human exomes. Nature 2009, 461(7261):272-276.

[123] Lessons from the Cancer Genome. Levi A. Garraway, Eric S. Lander. Cell - 28 March 2013 Vol. 153, Issue 1, pp. 17-37. 


\section{VITA}

\section{JUAN CARLOS MARTINEZ}

\section{PUBLICATIONS, ABSTRACTS AND PRESENTATIONS}

\section{Journals}

Juan Carlos Martinez, Nelson Lopez-Jimenez, Tao Meng, and S. S. Iyengar. Predicting DNA Mutations during Cancer Evolution, accepted for publication. International Journal of Bioinformatics Research and Applications.

Juan Carlos Martinez and Tao Meng. A Modeling Approach for the Prediction of Mutation in Genomic Sequences, accepted for publication. International Journal of Computer Science.

Juan Carlos Martinez, S. S. Iyengar, and Nelson Lopez-Jimenez. Toward the prediction of mutations in genomic sequences from patients with cancer. Journal of Clinical Bioinformatics. (Under review).

\section{Conference/Poster Presentations}

Juan Carlos Martinez, S. S. Iyengar, Nelson Lopez-Jimenez. A computational approach toward predicting gene mutations by genome-wide sequencing analysis of both normal and cancer cells from the same individual. Beyond the Genome 2013. San Francisco, CA, October 1-3, 2013.

Juan Carlos Martinez, S. S. Iyengar, Nelson Lopez-Jimenez. A model for predicting gene mutations in the genome of individuals with AML during disease evolution by genome-wide sequencing analysis. The American Society for Cell Biology (ASCB 2013). New Orleans, LA, December 14-18, 2013.

Juan Carlos Martinez, S. S. Iyengar, Nelson Lopez-Jimenez. Predicting gene mutations during cancer evolution: a new tool in searching novel targets for cancer treatment.. 11th Annual Rocky Mountain Bioinformatics Conference. Aspen/Snowmass, Colorado, December 12-14, 2013. 


\section{Other Publications}

Juan Carlos Martinez, Lixi Wang, S. Masoud Sadjadi, Ming Zhao, Experimental Study of Large-scale Computing on Virtualized Resources. VTDC '09 Proceedings of the 3rd International Workshop on Virtualization Technologies in Distributed Computing.

Hector A. Duran Limon, S. Masoud Sadjadi, Raju Rangaswami, Shu Shimizu, Liana Fong, Rosa M. Badia, Pat Welsh, Sandeep Pattnaik, Anthony Praino, Javier Figueroa, Javier Delgado, Xabriel J. Collazo-Mojica, David Villegas, Selim Kalayci, Gargi Dasgupta, Onyeka Ezenwoye, Khalid Saleem, Juan Carlos Martinez, Ivan Rodero, Shuyi Chen, Javier Muñoz, Diego Lopez, Julita Corbalan, Hugh Willoughby, Michael McFail, Christine Lisetti, and Malek Adjouadi. Grid enablement and resource usage prediction of weather research and forecasting. In Proceedings of the Collaborative and Grid Computing Technologies Workshop, page 4, Cancun, Mexico, April 2008.

Yanbin Liu, S. Masoud Sadjadi, Liana Fong, Ivan Rodero, David Villegas, Selim Kalayci, Norman Bobroff, and Juan Carlos Martinez. Enabling autonomic metascheduling in grid environments. In Proceedings of the 5th IEEE International Conference on Autonomic Computing (ICAC-2008), pages 199-200, Chicago, IL, June 2008.

Norman Bobroff, Liana Fong, Selim Kalayci, Yanbin Liu, Juan Carlos Martinez, Ivan Rodero, S. Masoud Sadjadi, and David Villegas. Enabling interoperability among metaschedulers. In Proceedings of 8th IEEE International Symposium on Cluster Computing and the Grid (CCGrid-2008), pages 306-315, Lyon, France, 2008.

S. Masoud Sadjadi, Liana Fong, Rosa M. Badia, Javier Figueroa, Javier Delgado, Xabriel J. Collazo-Mojica, Khalid Saleem, Raju Rangaswami, Shu Shimizu, Hector A. Duran Limon, Pat Welsh, Sandeep Pattnaik, Anthony Praino, David Villegas, Selim Kalayci, Gargi Dasgupta, Onyeka Ezenwoye, Juan Carlos Martinez, Ivan Rodero, Shuyi Chen, Javier Muñoz, Diego Lopez, Julita Corbalan, Hugh Willoughby, Michael McFail, Christine Lisetti, and Malek Adjouadi. Transparent grid enablement of weather research and forecasting. In Proceedings of the Mardi Gras Conference 2008 - Workshop on GridEnabling Applications, Baton Rouge, Louisiana, USA, January 2008.

S. Masoud Sadjadi, J. Martinez, T. Soldo, L. Atencio, R. M. Badia, and J. Ejarque. Improving separation of concerns in the development of scientific applications. In Proceedings of The Nineteenth International Conference on Software Engineering and Knowledge Engineering (SEKE'2007), pages 456-461, Boston, USA, July 2007.

Tao Li, S. Masoud Sadjadi, Juan Carlos Martinez, Lokesh Sasikumar, and Manoj Pillai. Data mining for autonomic system management: A case study at fiu-scis. Technical report, Florida International University, 2006. Technical Report: FIU-SCIS-2006-03-01. 